\title{
Ventriculo-peritoneal shunting devices for hydrocephalus (Review)
}

Garegnani L, Franco JVA, Ciapponi A, Garrote V, Vietto V, Portillo Medina SA

Garegnani L, Franco JVA, Ciapponi A, Garrote V, Vietto V, Portillo Medina SA. Ventriculo-peritoneal shunting devices for hydrocephalus.

Cochrane Database of Systematic Reviews 2020, Issue 6. Art. No.: CD012726.

DOI: 10.1002/14651858.CD012726.pub2.

www.cochranelibrary.com 
TABLE OF CONTENTS

HEADER 1

ABSTRACT

PLAIN LANGUAGE SUMMARY

SUMMARY OF FINDINGS

BACKGROUND

OBJECTIVES

METHODS

RESULTS

Figure 1.

Figure 2.

Figure 3.

DISCUSSION

AUTHORS' CONCLUSIONS

ACKNOWLEDGEMENTS

REFERENCES

CHARACTERISTICS OF STUDIES

DATA AND ANALYSES

Analysis 1.1. Comparison 1: Standard valve vs anti-syphon valve, Outcome 1: Mortality

Analysis 2.1. Comparison 2: Different types of standard valves, Outcome 1: Treatment failure

Analysis 2.2. Comparison 2: Different types of standard valves, Outcome 2: Mortality

Analysis 3.1. Comparison 3: Standard valve versus constant flow valve, Outcome 1: Treatment failure

Analysis 4.1. Comparison 4: Programmable valve versus non-programmable valve, Outcome 1: Treatment failure

Analysis 4.2. Comparison 4: Programmable valve versus non-programmable valve, Outcome 2: Adverse events APPENDICES

HISTORY

CONTRIBUTIONS OF AUTHORS

DECLARATIONS OF INTEREST

SOURCES OF SUPPORT

DIFFERENCES BETWEEN PROTOCOL AND REVIEW 
[Intervention Review]

\section{Ventriculo-peritoneal shunting devices for hydrocephalus}

Luis Garegnani ${ }^{1}$, Juan VA Franco ${ }^{2}$, Agustín Ciapponi ${ }^{3}$, Virginia Garrote ${ }^{4}$, Valeria Vietto5, Santiago Adalberto Portillo Medina6

1Research Department, Instituto Universitario Hospital Italiano, Buenos Aires, Argentina. ${ }^{2}$ Argentine Cochrane Centre, Instituto Universitario Hospital Italiano, Buenos Aires, Argentina. ${ }^{3}$ Argentine Cochrane Centre, Institute for Clinical Effectiveness and Health Policy (IECS-CONICET), Buenos Aires, Argentina. ${ }^{4}$ Biblioteca Central, Instituto Universitario Hospital Italiano, Buenos Aires, Argentina. ${ }^{5}$ Family and Community Medicine Service, Hospital Italiano de Buenos Aires, Buenos Aires, Argentina. ${ }^{6}$ Pediatric Neurosurgery Service, Hospital Italiano de Buenos Aires, Buenos Aires, Argentina

Contact address: Santiago Adalberto Portillo Medina, santiago.portillo@hospitalitaliano.org.ar.

Editorial group: Cochrane Multiple Sclerosis and Rare Diseases of the CNS Group.

Publication status and date: New, published in Issue 6, 2020.

Citation: Garegnani L, Franco JVA, Ciapponi A, Garrote V, Vietto V, Portillo Medina SA. Ventriculo-peritoneal shunting devices for hydrocephalus. Cochrane Database of Systematic Reviews 2020, Issue 6. Art. No.: CD012726. DOI: 10.1002/14651858.CD012726.pub2.

Copyright ( 2020 The Cochrane Collaboration. Published by John Wiley \& Sons, Ltd.

\section{A B S T R A C T}

\section{Background}

Hydrocephalus is a common neurological disorder, caused by a progressive accumulation of cerebrospinal fluid (CSF) within the intracranial space that can lead to increased intracranial pressure, enlargement of the ventricles (ventriculomegaly) and, consequently, to brain damage. Ventriculo-peritoneal shunt systems are the mainstay therapy for this condition, however there are different types of shunt systems.

\section{Objectives}

To compare the effectiveness and adverse effects of conventional and complex shunt devices for CSF diversion in people with hydrocephalus.

\section{Search methods}

We searched the Cochrane Central Register of Controlled Trials (2020 Issue 2); Ovid MEDLINE (1946 to February 2020); Embase (Elsevier) (1974 to February 2020); Latin American and Caribbean Health Science Information Database (LILACS) (1980 to February 2020); ClinicalTrials.gov; and World Health Organization International Clinical Trials Registry Platform.

\section{Selection criteria}

We selected randomised controlled trials or quasi-randomised trials of different types of ventriculo-peritoneal shunting devices for people with hydrocephalus. Primary outcomes included: treatment failure, adverse events and mortality.

\section{Data collection and analysis}

Two review authors screened studies for selection, assessed risk of bias and extracted data. Due to the scarcity of data, we performed a Synthesis Without Meta-analysis (SWiM) incorporating GRADE for the quality of the evidence.

\section{Main results}

We included six studies with 962 participants assessing the effects of standard valves compared to anti-syphon valves, other types of standard valves, self-adjusting CSF flow-regulating valves and external differential programmable pressure valves. All included studies started in a hospital setting and offered ambulatory follow-up. Most studies were conducted in infants or children with hydrocephalus from diverse causes. The certainty of the evidence for most comparisons was low to very low.

\section{Standard valve versus anti-syphon valve}


Three studies with 296 randomised participants were included under this comparison. We are uncertain about the incidence of treatment failure in participants with standard valve and anti-syphon valves (very low certainty of the evidence). The incidence of adverse events may be similar in those with standard valves (range 0 to $1.9 \%$ ) and anti-syphon valves (range 0 to $2.9 \%$ ) (low certainty of the evidence). Mortality may be similar in those with standard valves ( $0 \%)$ and anti-syphon valves $(0.9 \%)(\mathrm{RD} 0.01 \%, 95 \% \mathrm{Cl}-0.02 \%$ to $0.03 \%$, low certainty of the evidence). Ventricular size and head circumference may be similar in those with standard valves and anti-syphon valves (low certainty of the evidence). None of the included studies reported the quality of life of participants.

\section{Comparison between different types of standard valves}

Two studies with 174 randomised participants were included under this comparison. We are uncertain about the incidence of treatment failure in participants with different types of standard valves (early postoperative period: $\mathrm{RR} 0.41,95 \% \mathrm{Cl} 0.13$ to 1.27 ; at 12 months followup: RR $1.17,95 \% \mathrm{Cl} 0.72$ to 1.92 , very low certainty of the evidence). None of the included studies reported adverse events beyond those included under "treatment failure". We are uncertain about the effects of different types of standard valves on mortality (range $2 \%$ to $17 \%$, very low certainty of the evidence). The included studies did not report the effects of these interventions on quality of life, ventricular size reduction or head circumference.

\section{Standard valve versus self-adjusting CSF flow-regulating valve}

One study with 229 randomised participants addressed this comparison. The incidence of treatment failure may be similar in those with standard valves (42.98\%) and self-adjusting CSF flow-regulating valves (39.13\%) (low certainty of the evidence). The incidence of adverse events may be similar in those with standard valves (range 0 to 1.9\%) and those with self-adjusting CSF flow-regulating valves (range 0 to 7.2\%) (low certainty of the evidence). The included study reported no deaths in either group in the postoperative period. Beyond the early postoperative period, the authors stated that nine patients died (no disaggregated data by each type of intervention was available, low certainty of the evidence). The included studies did not report the effects of these interventions on quality of life, ventricular size reduction or head circumference.

\section{External differential programmable pressure valve versus non-programmable valve}

One study with 377 randomised participants addressed this comparison. The incidence of treatment failure may be similar in those with programmable valves (52\%) and non-programmable valves (52\%) (RR 1.02, $95 \% \mathrm{Cl} 0.84$ to 1.24 , low certainty of the evidence). The incidence of adverse events may be similar in those with programmable valves $(6.19 \%)$ and non-programmable valves (6.01\%) (RR $0.97,95 \% \mathrm{Cl} 0.44$ to 2.15 , low certainty of the evidence). The included study did not report the effect of these interventions on mortality, quality of life or head circumference. Ventricular size reduction may be similar in those with programmable valves and non-programmable valves (low certainty of the evidence).

\section{Authors' conclusions}

Standard shunt valves for hydrocephalus compared to anti-syphon or self-adjusting CSF flow-regulating valves may cause little to no difference on the main outcomes of this review, however we are very uncertain due to the low to very low certainty of evidence. Similarly, different types of standard valves and external differential programmable pressure valves versus non-programmable valves may be associated with similar outcomes. Nevertheless, this review did not include valves with the latest technology, for which we need highquality randomised controlled trials focusing on patient-important outcomes including costs.

\section{PLAIN LANGUAGE SUMMARY}

\section{Ventriculo-peritoneal valves for hydrocephalus}

\section{Review question}

What are the effects (benefits and harms) of different types of shunt devices for people being treated for hydrocephalus?

\section{Background}

Hydrocephalus, known as 'water on the brain' is a disorder caused by the accumulation of cerebrospinal fluid in the cavities (ventricles) deep within the brain. This causes an increase in the size of the ventricles and pressure on the brain that leads to brain damage. Ventriculoperitoneal valves are devices that drain the extra fluid from the brain into the peritoneal cavity,in the abdomen, where the fluid can be absorbed (a ventriculo-peritoneal shunt). There are different types of valves and the different effects are unknown.

\section{Study characteristics}

We searched for the evidence up to February 2020. We included six studies with 962 participants evaluating: anti-syphon valves, different types of standard valves, self-adjusting cerebrospinal fluid flow-regulating valves and programmable valves. Most of the studies included children with hydrocephalus with a follow-up between two to six years. Four studies did not specify their funding sources. One study was funded by the manufacturer of the device and another study was funded by a foundation. 


\section{Key results}

We are uncertain about the differences in treatment failure between standard valves and anti-syphon valves, however, the incidence of adverse events, mortality, ventricular size and head circumference may be similar. None of the included studies reported the quality of life of participants.

We are uncertain about the differences in treatment failure and mortality in participants with different types of standard valves. The included studies did not report the effects of these interventions on quality of life, ventricular size and head circumference.

The incidence of treatment failure and adverse events may be similar in those with standard valves and self-adjusting cerebrospinal fluid flow-regulating valves. The included study reported no deaths in either group early after the operation; we are uncertain about effects beyond this period. The included studies did not report the effects of these interventions on quality of life, ventricular size reduction or head circumference.

The incidence of treatment failure is probably similar in those with programmable valves and non-programmable valves, and the incidence of adverse events and ventricular size may be also similar. The included study did not report the effect of these interventions on mortality, quality of life or head circumference.

\section{Quality of the evidence}

The certainty of the evidence was mostly low to very low since the studies were poorly conducted, with a small number of participants. Furthermore, many studies did not report critical outcomes such as mortality. 


\section{SUMMARY OF FINDINGS}

\section{Summary of findings 1. Standard valve compared to anti-syphon valve for hydrocephalus}

\section{Standard valve compared to anti-syphon valve for hydrocephalus}

Patient or population: participants with hydrocephalus

Setting: hospital procedure - outpatient follow-up (Iran, USA, Canada and France, Switzerland)

Intervention: Standard valve

Comparison: Anti-syphon valve

\begin{tabular}{|c|c|c|c|}
\hline Outcomes & Impact & $\begin{array}{l}\text { № of partici- } \\
\text { pants } \\
\text { (studies) }\end{array}$ & $\begin{array}{l}\text { Certainty } \\
\text { of the evi- } \\
\text { dence } \\
\text { (GRADE) }\end{array}$ \\
\hline $\begin{array}{l}\text { Treatment failure: morbidity asso- } \\
\text { ciated with shunt placement that } \\
\text { led to shunt revision }\end{array}$ & $\begin{array}{l}\text { There is uncertainty about the incidence of treatment failure } \\
\text { in participants with standard valve compared to anti-syphon } \\
\text { valves. }\end{array}$ & $\begin{array}{l}296 \\
\text { (3 RCTs) }\end{array}$ & $\begin{array}{l}\oplus \odot \Theta \ominus \\
\text { VERY LOW } 12\end{array}$ \\
\hline Follow-up: 2 to 6 years & $\begin{array}{l}\text { Range with standard valve: } 43-55 \% \\
\text { Range with anti-syphon valve: } 49-50 \%\end{array}$ & & \\
\hline $\begin{array}{l}\text { Adverse events: morbidity related } \\
\text { to the shunt placement that has not } \\
\text { led to shunt revision } \\
\text { Follow-up: } 2 \text { to } 6 \text { years }\end{array}$ & $\begin{array}{l}\text { The incidence of adverse events may be similar in those with } \\
\text { the standard valves compared to anti-syphon valves. } \\
\text { Range with standard valve: } 0-1.8 \% \text { for each type of adverse } \\
\text { event } \\
\text { Range with anti-syphon valve: } 0-2.8 \% \text { for each type of ad- } \\
\text { verse event }\end{array}$ & $\begin{array}{l}229 \\
(1 \mathrm{RCT})\end{array}$ & $\begin{array}{l}\oplus \oplus \odot \ominus \\
\text { LOW } 34\end{array}$ \\
\hline $\begin{array}{l}\text { Mortality } \\
\text { In the early postoperative period ( } \leq \\
30 \text { days) }\end{array}$ & $\begin{array}{l}\text { Mortality may be similar in those with standard valves com- } \\
\text { pared to anti-syphon valves. } \\
\text { Mortality in standard valve: } 0 \% \\
\text { Mortality with anti-syphon valve: } 0.9 \% \\
\text { Risk difference } 0.01 \text { ( } 95 \% \mathrm{Cl}-0.02 \text { to } 0.03)\end{array}$ & $\begin{array}{l}229 \\
(1 \mathrm{RCT})\end{array}$ & $\begin{array}{l}\oplus \oplus \Theta \Theta \\
\text { LOW } 34\end{array}$ \\
\hline $\begin{array}{l}\text { Mortality } \\
\text { Beyond the early postoperative pe- } \\
\text { riod (> } 30 \text { days) }\end{array}$ & $\begin{array}{l}\text { We were unable to draw conclusions due to lack of disaggre- } \\
\text { gated data. }\end{array}$ & $\begin{array}{l}229 \\
(1 \mathrm{RCT})\end{array}$ & $\begin{array}{l}\oplus \oplus \ominus \ominus \\
\text { LOW } 34\end{array}$ \\
\hline Quality of life & Not reported & - & - \\
\hline
\end{tabular}

* The risk in the intervention group (and its $95 \%$ confidence interval) is based on the assumed risk in the comparison group and the relative effect of the intervention (and its $95 \% \mathrm{Cl}$ ).

Cl: Confidence interval; RR: Risk ratio.

\section{GRADE Working Group grades of evidence}

High certainty: We are very confident that the true effect lies close to that of the estimate of the effect

Moderate certainty: We are moderately confident in the effect estimate: The true effect is likely to be close to the estimate of the effect, but there is a possibility that it is substantially different

Low certainty: Our confidence in the effect estimate is limited: The true effect may be substantially different from the estimate of the effect 
Very low certainty: We have very little confidence in the effect estimate: The true effect is likely to be substantially different from the estimate of effect

1 Downgraded two levels due to high or unclear risk of bias in multiple domains of the included studies

2 Downgraded one level due to few participants and events in each study

3 Downgraded one level due to high and unclear risk of bias in multiple domains of the included study

4 Downgraded one level due to few participants and events in the included study

\section{Summary of findings 2 . Comparison between different types of standard valves}

\section{Comparison between different types of standard valves}

Patient or population: participants with hydrocephalus

Setting: hospital procedure - outpatient follow-up (Mexico and Uganda)

Intervention and comparison: distal cleft valve (Biomed), Hakim ball-in-cone and spring, Chhabra and Codman-Hakim

\begin{tabular}{|c|c|c|c|}
\hline Outcomes & Impact & $\begin{array}{l}\text { № of partici- } \\
\text { pants } \\
\text { (studies) }\end{array}$ & $\begin{array}{l}\text { Certainty } \\
\text { of the evi- } \\
\text { dence } \\
\text { (GRADE) }\end{array}$ \\
\hline $\begin{array}{l}\text { Treatment failure: } \\
\text { morbidity associated } \\
\text { with shunt placement } \\
\text { that led to shunt revi- } \\
\text { sion } \\
\text { Follow-up: see each } \\
\text { study }\end{array}$ & $\begin{array}{l}\text { There is uncertainty about the incidence of treatment failure in partici- } \\
\text { pants with different types of standard valves: } \\
\text { Garcia } 1988 \text { ( } 84 \text { participants) reported a similar incidence of early treat- } \\
\text { ment failure ( } 7 \% \text { vs } 21 \% \text { at } 30 \text { days) with the standard distal cleft valve } \\
\text { and the standard Hakim ball-in-cone and spring valve (RR } 0.41,95 \% \mathrm{Cl} \\
0.13 \text { to } 1.27 \text { ). } \\
\text { Warf } 2005 \text { ( } 90 \text { participants) reported a similar incidence of treatment } \\
\text { failure ( } 46 \% \text { vs } 42 \% \text { at } 12 \text { months) with the Chhabra valve and the Cod- } \\
\text { man-Hakim micro precision valve (RR } 1.17,95 \% \mathrm{Cl} 0.72 \text { to } 1.92 \text { ). }\end{array}$ & $\begin{array}{l}174 \\
\text { (2 RCTs) }\end{array}$ & $\begin{array}{l}\oplus \ominus \Theta \Theta \\
\text { VERY LOW } 12\end{array}$ \\
\hline $\begin{array}{l}\text { Adverse events: mor- } \\
\text { bidity related to the } \\
\text { shunt placement that } \\
\text { has not led to shunt re- } \\
\text { vision } \\
\text { Follow-up: not applica- } \\
\text { ble }\end{array}$ & $\begin{array}{l}\text { None of the included studies reported this outcome beyond those in- } \\
\text { cluded under "treatment failure". }\end{array}$ & (0 RCTs) & - \\
\hline $\begin{array}{l}\text { Mortality } \\
\text { In the early postopera- } \\
\text { tive period ( } \leq 30 \text { days) }\end{array}$ & $\begin{array}{l}\text { There is uncertainty about the effects of different types of standard } \\
\text { valves on mortality. } \\
\text { Warf } 2005 \text { reported a } 2 \% \text { mortality with the Chhabra valve and } 0 \% \text { with } \\
\text { the Codman-Hakim micro precision valve (RD } 0.02 \%, 95 \% \mathrm{Cl}-0.04 \% \text { to } \\
0.09 \%) \text {. } \\
\text { Garcia } 1988 \text { reported a } 9 \% \text { mortality with the standard distal cleft valve- } \\
\text { and } 0 \% \text { mortality with the standard } \mathrm{Hakim} \text { ball-in-cone and spring valve } \\
\text { in the early postoperative period (RD } 0.09 \%, 95 \% \mathrm{Cl}-0.00 \% \text { to } 0.18 \% \text { ). }\end{array}$ & $\begin{array}{l}174 \\
(2 \mathrm{RCT})\end{array}$ & $\begin{array}{l}\oplus \odot \Theta \Theta \\
\text { VERY LOW } 12\end{array}$ \\
\hline $\begin{array}{l}\text { Mortality } \\
\text { Beyond the early post- } \\
\text { operative period ( }>30 \\
\text { days) }\end{array}$ & $\begin{array}{l}\text { We are uncertain about the effects of different types of standard valves } \\
\text { on mortality > } 30 \text { days. } \\
\text { Warf } 2005 \text { reported a } 14 \% \text { mortality with the Chhabra valve and } 17 \% \text { with } \\
\text { the Codman-Hakim micro precision valve. }\end{array}$ & $\begin{array}{l}90 \\
(1 \mathrm{RCT})\end{array}$ & $\begin{array}{l}\oplus \Theta \Theta \Theta \\
\text { VERY LOW } 12\end{array}$ \\
\hline
\end{tabular}


*The risk in the intervention group (and its 95\% confidence interval) is based on the assumed risk in the comparison group and the relative effect of the intervention (and its $95 \% \mathrm{Cl}$ ).

CI: Confidence interval; RCT: randomised controlled trial; RD: Risk difference; RR: Risk ratio.

\section{GRADE Working Group grades of evidence}

High certainty: We are very confident that the true effect lies close to that of the estimate of the effect

Moderate certainty: We are moderately confident in the effect estimate: The true effect is likely to be close to the estimate of the effect, but there is a possibility that it is substantially different

Low certainty: Our confidence in the effect estimate is limited: The true effect may be substantially different from the estimate of the effect

Very low certainty: We have very little confidence in the effect estimate: The true effect is likely to be substantially different from the estimate of effect

1 Downgraded two levels due to high or unclear risk of bias in multiple domains of the included studies

2 Downgraded one level due to few participants and events in the included studies

\section{Summary of findings 3. Standard valve compared to self-adjusting CSF flow-regulating valve for hydrocephalus}

\section{Standard valve compared to self-adjusting CSF flow-regulating valve for hydrocephalus}

Patient or population: participants with hydrocephalus

Setting: inpatient procedure - outpatient follow-up (Canada, United States, France)

Intervention: standard valve

Comparison: self-adjusting CSF flow-regulating valve

\begin{tabular}{|c|c|c|c|}
\hline Outcomes & Impact & $\begin{array}{l}\text { № of partici- } \\
\text { pants } \\
\text { (studies) }\end{array}$ & $\begin{array}{l}\text { Certainty } \\
\text { of the evi- } \\
\text { dence } \\
\text { (GRADE) }\end{array}$ \\
\hline $\begin{array}{l}\text { Treatment failure: } \text { morbidity associ- } \\
\text { ated with shunt placement that led to } \\
\text { shunt revision }\end{array}$ & $\begin{array}{l}\text { The incidence of treatment failure may be similar in those } \\
\text { with the standard valve and the self-adjusting CSF flow- } \\
\text { regulating valve (RR } 1.08,95 \% \mathrm{Cl} 0.79 \text { to } 1.47 \text { ). }\end{array}$ & $\begin{array}{l}229 \\
(1 \mathrm{RCT})\end{array}$ & $\begin{array}{l}\oplus \oplus \Theta \Theta \\
\text { LOW } 12\end{array}$ \\
\hline Follow-up: 2 years & $\begin{array}{l}\text { Standard valve: } 42 \% \\
\text { Self-adjusting CSF flow-regulating valve: } 39 \%\end{array}$ & & \\
\hline $\begin{array}{l}\text { Adverse events: morbidity related to } \\
\text { the shunt placement that has not led } \\
\text { to shunt revision }\end{array}$ & $\begin{array}{l}\text { The incidence of adverse events may be similar in those } \\
\text { with the standard valve and those with the self-adjusting } \\
\text { CSF flow-regulating valve. }\end{array}$ & $\begin{array}{l}229 \\
(1 \mathrm{RCT})\end{array}$ & $\begin{array}{l}\oplus \oplus \Theta \Theta \\
\text { LOW } 12\end{array}$ \\
\hline Follow-up: 2 years & $\begin{array}{l}\text { Range with standard valve: } 0-1.9 \% \\
\text { Range with self-adjusting CSF flow-regulating valve: } 0 \text { - } \\
7.2 \%\end{array}$ & & \\
\hline $\begin{array}{l}\text { Mortality } \\
\text { In the early postoperative period }(\leq 30 \\
\text { days) }\end{array}$ & $\begin{array}{l}\text { The included studies reported no events in this period } \\
\text { (deaths). }\end{array}$ & $\begin{array}{l}229 \\
(1 \mathrm{RCT})\end{array}$ & $\begin{array}{l}\oplus \oplus \Theta \Theta \\
\text { LOW } 12\end{array}$ \\
\hline $\begin{array}{l}\text { Mortality } \\
\text { Beyond the early postoperative period } \\
\text { (> } 30 \text { days) }\end{array}$ & $\begin{array}{l}\text { Nine patients died. No disaggregated data by each type of } \\
\text { intervention were available. }\end{array}$ & $\begin{array}{l}229 \\
(1 \mathrm{RCT})\end{array}$ & $\begin{array}{l}\oplus \oplus \odot \ominus \\
\text { LOW } 12\end{array}$ \\
\hline
\end{tabular}


*The risk in the intervention group (and its 95\% confidence interval) is based on the assumed risk in the comparison group and the relative effect of the intervention (and its $95 \% \mathrm{Cl}$ ).

CI: Confidence interval; CSF: cerebrospinal fluid; $\mathbf{R C T}$ : randomised controlled trial; RR: Risk ratio.

\section{GRADE Working Group grades of evidence}

High certainty: We are very confident that the true effect lies close to that of the estimate of the effect

Moderate certainty: We are moderately confident in the effect estimate: The true effect is likely to be close to the estimate of the effect, but there is a possibility that it is substantially different

Low certainty: Our confidence in the effect estimate is limited: The true effect may be substantially different from the estimate of the effect

Very low certainty: We have very little confidence in the effect estimate: The true effect is likely to be substantially different from the estimate of effect

1 Downgraded one level due to high or unclear risk of bias in multiple domains in the included study

2 Downgraded one level due to few participants and events in the included study

\section{Summary of findings 4. External differential programmable pressure valve compared to non-programmable valves}

\section{External differential programmable pressure valve compared to non-programmable valves for hydrocephalus}

Patient or population: participants with hydrocephalus

Setting: inpatient treatment - outpatient follow-up (USA)

Intervention: external differential programmable pressure valve

Comparison: other types of valve (any non-programmable valve pressure fixed pressure available on the market: Delta (Medtronic PS Medical, Goleta Ca): 10\%, other Medtronic PS Medical: 57\%, other Codman/Johnson \& Johnson: 8\%, Orbis-Sigma (Cordis, Miami Lakes, $\mathrm{FL}): 3 \%$, others: $22 \%$ )

\begin{tabular}{|c|c|c|c|}
\hline Outcomes & Impact & $\begin{array}{l}\text { № of partici- } \\
\text { pants } \\
\text { (studies) }\end{array}$ & $\begin{array}{l}\text { Certainty } \\
\text { of the evi- } \\
\text { dence } \\
\text { (GRADE) }\end{array}$ \\
\hline $\begin{array}{l}\text { Treatment failure: morbidity associated with } \\
\text { shunt placement that led to shunt revision } \\
\text { Follow-up: } 2 \text { years }\end{array}$ & $\begin{array}{l}\text { The incidence of treatment failure is probably } \\
\text { similar in those with programmable valves and } \\
\text { the non-programmable valves (RR } 1.02,95 \% \mathrm{Cl} \\
0.84 \text { to } 1.24 \text { ). } \\
\text { Programmable valve: } 52 \% \\
\text { Other non-programmable fixed pressure valves: } \\
52 \%\end{array}$ & $\begin{array}{l}377 \\
(1 \mathrm{RCT})\end{array}$ & $\begin{array}{l}\oplus \oplus \Theta \Theta \\
\text { LOW } 12\end{array}$ \\
\hline $\begin{array}{l}\text { Adverse events: morbidity related to the } \\
\text { shunt placement that has not led to shunt revi- } \\
\text { sion } \\
\text { Follow-up: } 2 \text { years }\end{array}$ & $\begin{array}{l}\text { The incidence of adverse events may be similar } \\
\text { in those with programmable valves and non-pro- } \\
\text { grammable valves (RR } 0.97,95 \% \mathrm{Cl} 0.44 \text { to } 2.15 \text { ). } \\
\text { Programmable valve: } 6.19 \% \\
\text { Other non-programmable fixed pressure valves: } \\
6.01 \%\end{array}$ & $\begin{array}{l}377 \\
(1 \mathrm{RCT})\end{array}$ & $\begin{array}{l}\oplus \oplus \Theta \Theta \\
\text { LOW } 12\end{array}$ \\
\hline $\begin{array}{l}\text { Mortality } \\
\text { In the early postoperative period ( } \leq 30 \text { days) }\end{array}$ & Not reported & - & - \\
\hline Mortality & Not reported & - & - \\
\hline
\end{tabular}


Beyond the early postoperative period $(>30$ days)

\section{Quality of life}

Not reported

*The risk in the intervention group (and its 95\% confidence interval) is based on the assumed risk in the comparison group and the relative effect of the intervention (and its $95 \% \mathrm{Cl}$ ).

Cl: Confidence interval; $\mathbf{R C T}$ : randomised controlled trial; RR: Risk ratio

\section{GRADE Working Group grades of evidence}

High certainty: We are very confident that the true effect lies close to that of the estimate of the effect

Moderate certainty: We are moderately confident in the effect estimate: The true effect is likely to be close to the estimate of the effect, but there is a possibility that it is substantially different

Low certainty: Our confidence in the effect estimate is limited: The true effect may be substantially different from the estimate of the effect

Very low certainty: We have very little confidence in the effect estimate: The true effect is likely to be substantially different from the estimate of effect

1 Downgraded one level due to high or unclear risk of bias in multiple domains in the included study

2 Downgraded one level due to few participants and events in the included study 


\section{B A C K G R O U N D}

\section{Description of the condition}

A specialised fluid system circulates around the central nervous system, providing structural and nutritional support for the brain and spinal cord. The fluid is a modified filtrate of the blood called cerebrospinal fluid (CSF), and is produced by groups of ependymal cells, found in the brain and spinal cord, and concentrated in the four choroid plexuses situated in the cerebral ventricles (four normal cavities) of the brain (Champney 2016; Wolburg 2010). The total CSF volume at any time is approximately $150 \mathrm{~mL}$, of which 125 $\mathrm{mL}$ is produced inside the brain. The rate of production is $0.35 \mathrm{~mL}$ to $0.40 \mathrm{~mL}$ per minute. CSF flows from the lateral ventricles to the third ventricle and then through the aqueduct to the fourth ventricle. After that, it leaves the ventricular system through three foramina: two laterally placed (foramina of Luschka) and one medially placed (foramen of Magendie) to circulate in the subarachnoid space (which is a layer of connective tissue between dura matter and the brain surface) and, finally, it is absorbed into the venous system, through the arachnoid villi (an absorption system in the layer covering the brain) (Mancall 2011).

Hydrocephalus, known colloquially as 'water on the brain' or 'dropsy on the brain', is a common neurological disorder, caused by a progressive accumulation of CSF within the intracranial space that can lead to increased intracranial pressure, enlargement of the ventricles (ventriculomegaly) and, consequently, to brain damage. It results from problems with CSF production, circulation or reabsorption. The incidence of hydrocephalus in the USA lies between one and 32 cases per 10,000 births, depending on the definition used and the population studied (Jeng 2011; Kahle 2015). The most recent estimate of incidence comes from a large, population-based study of idiopathic (unknown cause) infantile hydrocephalus in Denmark over a 30-year period, which documented 1.1 cases per 1000 births (Munch 2012; Tully 2014).

Hydrocephalus can be classified into communicating and noncommunicating types on the basis of its pathophysiology. The non-communicating type, also called obstructive hydrocephalus, can appear in the early foetal stage as part of certain congenital malformations (such as aqueductal stenosis, Chiari malformation), or associated with a broad spectrum of brain malformations, which prevent the normal flow of CSF around the brain, and its subsequent reabsorption. Hydrocephalus may also occur secondary to an obstruction of the CSF circulation caused by a tumour located in the fluid-producing cavities of the brain (ventriculi). By comparison, communicating hydrocephalus results from a deficit in CSF reabsorption, for example, in postmeningitis hydrocephalus. Haemorrhage in preterm infants can cause hydrocephalus by two mechanisms: acute obstruction by a blood clot or a delayed reabsorption caused by thickening of arachnoids, the inner layer of the meninges (Petre 2010). Although infrequent, hydrocephalus can also be associated with an overproduction of CSF, as in the case of a choroid plexus papilloma, which is a type of benign intraventricular tumour.

Normal Pressure Hydrocephalus (NPH) is a condition first reported in adults that refers to a chronic symptomatic hydrocephalus with a normal CSF pressure (i.e. less than $18 \mathrm{mmHg}$ ) (Adams 1965). The condition is defined by a clinical triad of symptoms: dementia, gait difficulties, and urinary urge incontinence in the presence of ventriculomegaly documented by computed tomography (CT) or magnetic resonance imaging (MRI) (Ziebell 2013; Halperin 2015). There are signs in the magnetic resonance images that differentiate $\mathrm{NPH}$ from other types of hydrocephalus, specifically, the amount of interstitial oedema surrounding the lateral ventricles (which should be minimal or absent), and the presence of brain deep white matter ischaemia (inadequate blood flow) also known as small vessel ischaemia or leukoaraiosis (Bradley 2015). In order to avoid overlap with other Cochrane Reviews, this review did not include people with NPH (Esmonde 2002; Tudor 2015; Ziebell 2013).

Signs and symptoms of hydrocephalus depend on the cause of the condition and time of presentation. In congenital hydrocephalus, patients present at birth with macrocephaly (overly large head), scalp vein distension, a bulging fontanelle and diastasis of sutures (stretching of fibrous joints between bones in the skull). These can be correctly evaluated using reference tables developed by measuring the circumference of the head of many children at different ages that are considered to establish the normal parameters of head growth. Using this simple tool, rapid head growth as a result of hydrocephalus can be detected clinically (O'Neill 1961). Patients with hydrocephalus may also present with Parinaud's phenomenon, which is an inability to raise the eyes upwards, also known as 'Sunset Sign', caused by an increased pressure on the tectal plate, which is the area of the midbrain where the oculomotor nerve originates. Symptoms in patients with acquired hydrocephalus (obstructive, post-infectious, or posthaemorrhagic) constitute the intracranial hypertension syndrome which includes headache, vomiting and drowsiness (Petre 2010). The appearance of these signs and symptoms of intracranial hypertension is what differentiates developing hydrocephalus from benign ventriculomegaly (dilation of the lateral ventricles in the brain without causing an increase in the CSF pressure and, therefore, without risk of brain damage).

In addition to physical examination and assessment of symptoms, confirmation of diagnosis occurs by means of imaging studies that show enlargement of ventricles. For newborns and infants, ultrasonography is frequently used because it can be done at the bedside and is radiation-free. Nevertheless, it is an operatordependent technology and does not assess the posterior fossa (intracranial space occupied by the cerebellum and brain stem), which sometimes makes it difficult to establish the cause of the hydrocephalus. For older children and adults, CT or MRI of the brain is usually used (Carey 1994).

Since Hippocrates in the fifth century BC, a poor understanding of the pathophysiology of hydrocephalus contributed to the failure of the early, and occasional attempts at therapy (Whytt 1768). In 1908, Payr introduced the first ventriculo-venous shunt for drainage, which consisted of a vein graft that led from the ventricles directly into the sagittal sinus (a vein that surrounds the top of the brain from front to back) and jugular veins (Mccullough 1990). In the same year, Kaush used a rubber conduit to drain the lateral ventricle into the peritoneal cavity (Kausch 1908), but the medical community received this innovation with little enthusiasm. Research for more effective treatments followed, and eventually the placement of intracranial shunts was tested. Efforts to drain the excess CSF into other body cavities have also been considered; for example, Matson and colleagues at Boston Children's Hospital first reported a ureteral diversionary procedure (Matson 1949).

The early twentieth century was a period in which knowledge of hydrocephalus, its diagnosis, and strategies for treatment evolved. 
Between January 1938 and December 1957, an observational, case-series study, documented the progress of 182 patients with congenital or acquired (early after birth, due to trauma or infection) hydrocephalus, who were not operated on. By the end of the study, only 81 patients were alive, and these had spontaneously arrested hydrocephalus, that is, a gradual slowing of the rate of head growth with a reduction in fontanelle tension and improvement in the patient's general condition. Patients with comorbidities, such as myelomeningocele or Chiari malformation, had the worst prognosis (Laurence 1962). Since the introduction of an effective therapy for the symptoms of hydrocephalus in 1956, when the first shunt system became available, mortality rates have fallen from around $45 \%$ to $53 \%$ - depending on the case series concerned to 15\% (Hagberg 1962; Jansen 1985; Laurence 1967; Yashon 1963). Morbidity rates have also significantly improved, with up to $42 \%$ of treated hydrocephalus patients enjoying a normal lifestyle after receiving shunts (Hirsch 1994).

The breakthrough that launched the modern era of surgical treatment for hydrocephalus was the introduction of valveregulated shunts and biocompatible synthetic materials in 1952 (Lifshutz 2001). That same year, Nulsen, Spitz and Holter, reported the successful use of a ventriculo-jugular shunt regulated by a spring and ball valve (Drake 1995), and almost simultaneously, Pudenz and colleagues, created a silicone one-way slit valve (Pudenz 1957). The development of the valve system and the availability of new biocompatible materials made it possible to divert CSF safely and reliably, and to avoid the many complications associated with unregulated CSF drainage.

Despite this degree of innovation, these devices were not exempt from shunt failure, which represents a serious complication. The most common causes of shunt malfunction include over- and under-drainage, mechanical mismatch (Inadequate selection of the pressure in a programmable valve or inadequate selection of the valve in relation to the type of hydrocephalus), occlusion (blocking) of the shunt and valve failure. These problems are largely resolved by replacing the valve or parts of the shunt, and sometimes by finding alternative drainage locations (for example, using the circulatory system or the pleural cavity in patients in whom the peritoneal cavity can not be used because of peritoneal inflammatory diseases or surgical sequelae) as well as with innovations in valve designs (Stein 2008).

Although implant of ventriculo-peritoneal shunts is the standard treatment for patients with hydrocephalus, in the 1990s, an additional method to treat hydrocephalus without the use of a prosthetic device became available. Endoscopic third ventriculostomy (ETV) is a minimally invasive procedure originally introduced by Dandy in 1922. During the procedure, a neuroendoscope is introduced, through a burr hole, into the lateral cerebral ventricles and a hole is made within the third ventricle to enable intracerebral ventricles to communicate with the subarachnoid spaces. This procedure leads to a more physiological circulation of CSF and is a well established treatment for non-communicating hydrocephalus in some neurosurgical units (Schroeder 1999). Risk of harms related to treatment is always present, depending on the procedure. Shunts may have complications related to placement, such as brain haemorrhages, brain damage, infections, coma, and even exceptionally, death (Smith 2004). In addition, there is an important and permanent risk of shunt malfunction. On the other hand, ETV may also carry serious complications including vascular injuries, hypothalamic injury (brain damage), and occasional death, as has been reported (Drake 2006; Hader 2002). Due to the lack of clinical trials comparing both strategies, the decision about whether to use ETV or ventriculoperitoneal shunts remains a topic of considerable debate (Cheng 2015; Limbrick 2014,Texakalidis 2019).

\section{Description of the intervention}

This review focused on the placement of various types of ventriculo-peritoneal shunts for the treatment of hydrocephalus. We define a ventriculo-peritoneal shunt as a system composed of a ventricular catheter, which is usually inserted into one of the cerebral lateral ventricles and is attached to both a valve and a distal catheter (at the other end of the system), which is implanted within the peritoneal cavity (abdomen) where CSF is finally reabsorbed (ICD-9-CM 2004; Patwardhan 2005).

Ventriculo-peritoneal shunting is a complex surgical intervention. This procedure is usually performed by an experienced neurosurgeon, in the sterile conditions of an operating theatre, and with the use of general anaesthesia. In the most commonly used surgical technique, the patient lies on the operating table in the supine position (lying on his back) with his head turned to one side. Using sterile instruments, the surgeon makes two incisions. One is made on the scalp in order to expose the posterior parietal part of the skull (above and behind the ear), followed by a burr hole through the skull and a small opening in the dura (membrane that covers the brain), to access the dilated lateral ventricle with a catheter. The second incision is made in the skin of the abdomen; from there a tunnel is formed under the skin towards the skull incision, and through this tunnel the distal catheter is passed. This catheter is subsequently connected to the valve part of the shunt. The ventricular catheter is also connected to the valve, which, in turn, is fixed to the periosteum (connective tissue around the skull). Finally, the distal end of the abdominal catheter is placed into the peritoneal cavity, into which the CSF is drained (Warf 2005).

Valves are the most important part of the system, and there are a variety of valve systems available that offer fixed pressures, antisiphon devices, and programmable and self-adjusting CSF flowregulating capabilities (Ames 1967; Kaiser 1992; Lumenta 1990; Ojemann 1968).

Fixed differential pressure (DP) valves are considered to be the first generation of valves. When pressure builds up inside the tubing, a slit is forced to open and CSF is allowed to flow outwards. Only unidirectional flow is permitted since an increase in external pressure closes the slit. The opening pressure is determined by the thickness of tubing walls. They are commonly classified according to the opening and closure pressures: low pressure $\left(20 \mathrm{~mm} \mathrm{H}_{2} \mathrm{O}\right.$ to $40 \mathrm{~mm} \mathrm{H}_{2} \mathrm{O}$ ), medium pressure $\left(40 \mathrm{~mm} \mathrm{H}_{2} \mathrm{O}\right.$ to $70 \mathrm{~mm} \mathrm{H}_{2} \mathrm{O}$ ) and high pressure valves $\left(80 \mathrm{~mm} \mathrm{H}_{2} \mathrm{O}\right.$ to $100 \mathrm{~mm} \mathrm{H}_{2} \mathrm{O}$ ) (Post 1985)

Second-generation valves include DP valves with flow-regulating devices, valves with anti-siphon mechanisms and programmable DP valves. Flow-regulating devices limit CSF flow through the valve by progressively narrowing its orifice in response to increasing intracranial pressure (ICP), as a pressure sensitive ring moves along a variable-diameter rod. Its aim is to prevent both postural and vasogenic (mediated by blood vessels) over-drainage occurring during rapid eye movement (REM) sleep, physical exertion, coughing, and other physiological conditions (Hanlo 2003). 
Through an increase in flow resistance, anti-siphon devices counteract the effect of hydrostatic negative pressure when the patient stands in a vertical position. The aim of this system is to provide performance characteristics that simulate those of normal CSF absorption, while allowing the regulation of ICP in a fashion similar to that seen in healthy individuals when they change their head position from horizontal to vertical (Baird 2014; Watson 1994).

Programmable variable pressure valves allow the selection of different opening pressures (between $30 \mathrm{~mm} \mathrm{H}_{2} \mathrm{O}$ and $200 \mathrm{~mm} \mathrm{H}_{2} \mathrm{O}$ in intervals of $10 \mathrm{~mm} \mathrm{H}_{2} \mathrm{O}$ ). The opening pressure can be selected through a percutaneous noninvasive magnetic programmer that manages valve pressure according to the patient's clinical and radiological evolution in terms of ventricular size. This permits avoidance of over-drainage and its consequences, which include slit (shaped) ventricles (accompanied by headaches) and retardation of cranial vault growth (secondary craniosynostosis) (Baird 2014; Miyake 2016; Xu 2013).

Finally, third-generation valves include DP programmable and antisiphon or gravitational systems combined in the same device. The DP unit allows the selection of an opening pressure between 0 to 20 $\mathrm{Cm} \mathrm{H}_{2} \mathrm{O}$, with the possibility of a transcutaneous adjustment. Unlike anti-siphon systems, which use a fixed pressure, the gravitational unit can be set to opening pressures of $15 \mathrm{~cm} \mathrm{H}_{2} \mathrm{O}, 20 \mathrm{~cm} \mathrm{H}_{2} \mathrm{O}, 25$ $\mathrm{cm} \mathrm{H}_{2} \mathrm{O}$, or $30 \mathrm{~cm} \mathrm{H}_{2} \mathrm{O}$, which cannot be changed after placement. This kind of device increases the opening pressure of the shunt by blocking the inlet flow using a gravity-assisted ball bearing. These devices must be placed vertically in order to counteract the siphoning effect of negative hydrostatic pressures when the patient is standing (Rohde 2009).

\section{How the intervention might work}

The balance in production, circulation, and reabsorption of the CSF has a key role in the Monro-Kelly hypothesis (Champney 2016; Lee 2009). This states that a stable ICP is the result of a rigid sphere (cranium) occupied by a non-compressible volume of brain tissue, blood and cerebrospinal fluid, which must remain constant. Although physiological fluctuations can occur, these are related to reciprocal changes in the amount of blood and CSF at a given time (Han 2005). ICP varies during the day; normal values are between $7 \mathrm{mmHg}$ to $15 \mathrm{mmHg}$ in a supine adult. Fluctuations are regulated primarily by cranial venous system blood volumes (CVSBV). As a result of gravity, CVSBV diminish when a person is upright. A drop in blood volume causes a retraction in brain tissues away from the normal CSF filled ventricles, causing a drop in ICP. Production of CSF also fluctuates during the day, but, as there is a balance between production and reabsorption, the ventricular size remains stable. There are also variations in CSF flow rate at night, with periods of highest flow rate occurring during REM sleep (Watson 1994).

Normally, CSF is produced, circulates, and is reabsorbed within the craniospinal space. Changes in body position between the horizontal and vertical entail transfer of the CSF and blood from the cranial compartment to the spinal compartment in a balanced fashion. When a ventriculo-peritoneal shunt is in place in a person with hydrocephalus, this balance is broken and hydrostatic pressure plays an important role (Kurtom 2007). Valves provide a resistance mechanism within the shunt system, which serves to control ICP more than CSF flow in patients with hydrocephalus (Miyake 2016).
In the developmental stage, shunt systems were synonymous with CSF diversion to provide a rapid decrease in ICP, with very little attention given to the cause of hydrocephalus. This approach produced good early results in terms of resolution of ventricle enlargement and reduction of ICP, but complications related to over-drainage such as subdural haematomas (a type of blood clot that forms surrounding the brain) shunt failures due to catheter obstruction, multiple shunt revisions and infections did appear (Symss 2015).

Valve technology has evolved to demonstrate a more adequate understanding of the physiological balance that needs to be achieved in the production, circulation and reabsorption of CSF. As a result, modern valves decrease ICP, as well as reducing brain damage and shunt-related complications. Since the first fixed-pressure shunts, like those of Holter and Pudenz (Drake 1995; Pudenz 1957), shunt systems, and especially valves, have evolved to incorporate flow-regulating, anti-siphon and gravitational components that are designed to avoid the overdrainage that can be caused by changes in patient position (Czosnyka 1998).

\section{Why it is important to do this review}

Hydrocephalus is a common chronic neurological disease that places a significant burden both on individuals and society as a whole.The cost of its medical treatment is not completely understood, but appears to be increasing (Pikus 1997). The National Inpatient Sample (NIS) database, a nation-wide survey in USA healthcare facilities, identified that in 2000 the three most common causes of admission for hydrocephalus were: shunt malfunction (40.7\%), non-communicating hydrocephalus (16.6\%), and communicating hydrocephalus (13.2\%). The most common procedures that resulted from these hospitalisations were the placement of a shunt (43.4\%) and shunt replacement (42.8\%). The total costs of shunt-related procedures were estimated to be approximately one billion USD (Patwardhan 2005).

Additionaly, a longitudinal study between 1997 and 2003 found an increasing proportion of older children were admitted with shunt malfunctions, and, when hydrocephalus treatment and its complications were compared with other chronic illness, such as cystic fibrosis, the inpatient utilisation (admissions, length of stay and hospital charges) were higher for hydrocephalus (Shannon 2011).

In low- and middle-income countries, the treatment of hydrocephalus may encounter additional challenges due to the economic constraints that patients and families face to afford the costs of acquisition, transportation and access to proper care (Warf 2005).

Although ventriculo-peritoneal shunting has been the most widely used treatment for hydrocephalus in the twentieth century, it is unclear whether the outcomes of shunting have improved significantly over time, despite improvements in the understanding of CSF physiology and the technological advancements in valve design. The Hydrocephalus Clinical Research Network concluded that in comparison with patients treated in the 1990s, there has been a reduction in the risk of complications - assessed as the time to first shunt failure - by about $18 \%$ (Kulkarni 2013). However, the reasons for this improvement are not obvious, and thus, open to interpretation. 
Despite this, long-term health and quality of life (QoL) outcomes after shunt implant have been highly variable, and range from patients leading a near normal life to those with physical, cognitive, social and emotional impairments associated with disability. These outcomes rely not only on the cause of hydrocephalus, but also on treatment-related complications such as infections, haemorrhages leading to brain haematomas, brain injury due to Inadequate positioning of the catheter, coma, and occasionally, death (Smith 2004). They may appear shortly after surgery, and generally within six months of shunt implantation or revision (Kulkarni 2007; Peters 2014; Sciubba 2007).

The main goal of physicians, researchers and engineers when developing a new valve technology is to improve clinical outcomes - both physical and mental - as well as reducing the likelihood of shunt failure. A Cochrane systematic review published in 2013 , evaluated the effectiveness of flow-regulated versus differential pressure-regulated shunt valves for adults with normal pressure hydrocephalus (Ziebell 2013), but it was limited to two types of valves in a specific group of participants. These reasons, and the fast pace of technological progress, prompt the need for a highquality synthesis of the evidence for the effectiveness and safety of common, fixed-pressure and other more complex ventriculoperitoneal shunt devices for people with hydrocephalus with different causes.

\section{O B JECTIVES}

To compare the effectiveness and adverse effects of conventional and complex shunt devices for CSF diversion in people with hydrocephalus.

\section{METHODS}

\section{Criteria for considering studies for this review}

\section{Types of studies}

We included randomised controlled trials and quasi-randomised controlled trials. We included studies regardless of their publication status or language of publication.

\section{Types of participants}

People with clinical and imaging diagnosis of communicating or non communicating, congenital or acquired hydrocephalus, irrespective of cause or subtype.

No restrictions were made with respect to age, except for exclusion of adults considered to have normal pressure hydrocephalus due to clinical symptoms (dementia, gait dyspraxia, and incontinence) and radiological signs (ventricular enlargement disproportionate to the degree of cerebral atrophy), to prevent overlapping with other Cochrane Reviews (Esmonde 2002; Tudor 2015; Ziebell 2013).

\section{Types of interventions}

We included studies with head-to-head comparisons between the different type of devices for ventriculo-peritoneal shunting listed below:

- programmable valves: these allow selection of an opening pressure value and are regulated by an external device, with or without an anti-siphon element;
- gravitational (gravity-assisted) valves: these combine a differential pressure unit that allows the selection of an opening pressure between 0 and $20 \mathrm{~cm} \mathrm{H}_{2} \mathrm{O}$, with a gravitational unit to counteract siphoning, while the opening pressure can be regulated using telemetry;

- valves with an anti-siphon device: these counteract the effect of hydrostatic negative pressure when the patient's body is in the vertical position, through flow resistance;

- flow-regulating valves: this type of valve adaptively limits CSF flow through the valve by narrowing its orifice progressively in response to increasing ICP, to avoid the occurrence of both postural and vasogenic over-drainage during normal physiological conditions;

- conventional (standard) valve: CSF is drained only when ICP rises above the fixed valve pressure.

\section{Types of outcome measures}

We did not use measurement of the outcomes assessed in this review as eligibility criteria.

\section{Primary outcomes}

- Treatment failure: defined as morbidity associated with shunt placement (slit ventricles (i.e. over-drainage), obstruction or infections)) or progression of hydrocephalus detected by signs or symptoms of intracranial hypertension or imaging studies that led to shunt revision in the early postoperative period $(\leq 30$ days) or beyond the early postoperative period ( $>30$ days).

- Adverse events: morbidity related to the shunt placement that has not led to shunt revision (e.g. subdural haematomas, secondary craniosynostosis and microcephaly) in the early postoperative period ( $\leq 30$ days) or beyond the early postoperative period ( $>30$ days).

- Mortality: defined as death for all causes in the early postoperative period ( $\leq 30$ days) or beyond the early postoperative period ( $>30$ days).

\section{Secondary outcomes}

- Quality of life and health-related quality of life measured according to a validated questionnaire, such as: the Medical Outcomes Study (MOS) 36-Item Short-Form Health Survey (SF-36) (Ware 2012), and EuroQOL-5D (EQ-5D) for adults (Williams 1990), and the Child Health Questionnaire (CHQ) (Landgraf 1999), or the Hydrocephalus Outcome Questionnaire (HOQ) for children (Kulkarni 2004) in the early postoperative period ( $\leq 30$ days) or beyond the early postoperative period (> 30 days).

- Ventricular size reduction measured by cranial sonography or CT scan in the early postoperative period ( $\leq 30$ days) or beyond the early postoperative period ( $>30$ days).

- Head circumference measured before and after placement of the ventriculo-peritoneal shunt (occipitofrontal head circumference) in the early postoperative period ( $\leq 30$ days) or beyond the early postoperative period (> 30 days).

\section{Search methods for identification of studies}

We performed a comprehensive search with no restrictions on the language of publication or publication status. 


\section{Electronic searches}

We searched the following sources for eligible reports in any language (Appendix 1):

- Cochrane Central Register of Controlled Trials (CENTRAL; most recent issue);

- Ovid MEDLINE (1946 to February 2020);

- Elsevier/Embase (1974 to February 2020);

- Latin American and Caribbean Health Sciences Literature database (LILACS; 1980 to February 2020);

- ClinicalTrials.gov (clinicaltrials.gov) (inception to 12.02.2020); and

- WHO International Clinical Trials Registry Platform (apps.who.int/trialsearch/) (inception to 12.02.2020).

We combined the Ovid MEDLINE search with the Cochrane Highly Sensitive Search Strategy for identifying randomised trials in MEDLINE, Ovid format (Lefebvre 2011).

This search strategy was adapted to search CENTRAL, Embase and LILACS. See Differences between protocol and review.

\section{Searching other resources}

We identified other potentially eligible trials or ancillary publications by searching the reference lists of retrieved included trials, reviews, meta-analyses and health technology assessment reports. We also contacted study authors of included trials to identify any further studies that we may have missed. We contacted drug and device manufacturers for ongoing or unpublished trials. We also examined any relevant retraction statements and errata for included studies.

To identify additional studies, we reviewed proceedings of the annual meetings of the European Society of Pediatric Neurosurgery (ESPN) and the International Society of Pediatric Neurosurgery (ISPN) for the last three years (Appendix 2).

We also searched the source of grey literature, Open Grey (www.opengrey.eu) (1980 to February 2020) (Appendix 1). See Differences between protocol and review.

\section{Data collection and analysis}

\section{Selection of studies}

Independently, two review authors (SP, VV, or JVAF) scanned the abstract, title, or both, of the records retrieved, to determine which studies needed to be assessed further. Two review authors (SP, VV, or JVAF) investigated all potentially relevant records as full text, mapped records to studies, and classified studies as included studies, excluded studies, studies awaiting classification, or ongoing studies in accordance with the criteria for each provided in the Cochrane Handbook for Systematic Reviews of Interventions (Higgins 2011a). We resolved any discrepancies through consensus or recourse to a third review author (AC). If resolution of a disagreement was not possible, we designated the study as 'awaiting classification' and we contacted study authors for clarification. We documented reasons for exclusion of studies that may have reasonably been expected to be included in the review in a 'Characteristics of excluded studies' table. We presented an adapted Preferred Reporting Items for Systematic Reviews and
Meta-Analyses (PRISMA) flow diagram showing the process of study selection (Liberati 2009).

\section{Data extraction and management}

We developed a dedicated data abstraction form that we tested ahead of time.

For studies that fulfilled the inclusion criteria, two review authors (LIG, SP, VV, or JVAF) independently abstracted the following information, which we provided in the 'Characteristics of included studies' table.

- Study design.

- Study dates (if dates were not available then this was reported).

- Study settings and country.

- Participant inclusion and exclusion criteria.

- Participant details, baseline demographics.

- The number of participants by study and by study arm.

- Details of relevant experimental and comparator interventions such as: manufacturer, device model and characteristics.

- Definitions of relevant outcomes, and method and timing of outcome measurement, as well as any relevant subgroups.

- Sources of study funding.

- Declarations of interest by primary investigators.

We extracted outcome data relevant to this Cochrane Review as needed for calculation of summary statistics and measures of variance. For dichotomous outcomes, we attempted to obtain numbers of events and totals in order to populate a $2 \times 2$ table, as well as summary statistics with corresponding measures of variance. For continuous outcomes, we attempted to obtain means and standard deviations or data necessary to calculate this information. For time-to-event outcomes, we attempted to obtain hazard ratios (HR) with corresponding measures of variance or data necessary to calculate this information.

We resolved any disagreements by consensus, or, if required, by consultation with a third review author (AC).

We provided information, including trial identifiers, about potentially relevant ongoing studies in the table 'Characteristics of ongoing studies'.

We attempted to contact authors of included studies to obtain key missing data, as needed.

\section{Assessment of risk of bias in included studies}

Two review authors (SP, LIG, VV, or JVAF) assessed the risk of bias of each included study independently. We resolved disagreements by consensus, or by consultation with a third review author (AC).

We assessed risk of bias using the Cochrane 'Risk of bias' assessment tool (Higgins 2011b). We assessed the following domains:

- sequence generation (selection bias);

- allocation concealment (selection bias);

- blinding of participants and personnel (performance bias);

- blinding of outcome assessment (detection bias);

- incomplete outcome data (attrition bias); 
- selective outcome reporting (reporting bias); and

- other sources of bias.

We judged risk of bias domains as 'low risk', 'high risk' or 'unclear risk' and evaluated individual bias items as described in the Cochrane Handbook for Systematic Reviews of Interventions (Higgins 2011b). We presented a 'Risk of bias' summary figure to illustrate these findings.

For performance bias (blinding of participants and personnel) and detection bias (blinding of outcome assessment), we evaluated the risk of bias separately for each outcome, and, when we reported our findings in the 'Risk of bias' tables, we grouped outcomes according to whether they were measured subjectively or objectively.

We also assessed attrition bias (incomplete outcome data) on an outcome-specific basis, and grouped outcomes with like judgements when reporting our findings in the 'Risk of bias' tables.

In addition, we summarised the risk of bias across domains for each outcome in each included study, as well as across studies and domains for each outcome.

\section{Measures of treatment effect}

We expressed dichotomous data as risk ratios (RRs) with 95\% confidence intervals (CIs). We reported risk differences (RDs) when absolute risks in both groups were $<1 \%$. We expressed continuous data as mean differences (MDs) with $95 \% \mathrm{Cl}$, unless different studies used different measures to assess the same outcome, in which case we would have analysed data as standardised mean differences (SMDs) with $95 \% \mathrm{Cl}$ (re-expressed and presented as units of a familiar measure). We expressed time-to-event data as hazard ratios (HRs) with $95 \% \mathrm{Cl}$.

\section{Unit of analysis issues}

The unit of analysis was the individual participant. If a patient received more than one shunt during the course of a trial, we considered the outcomes related to the first implanted device only. If we had identified cluster-randomised trials, we would have followed the guidance of the Handbook Section 23.1.3 (Higgins 2019). Cross-over studies were unlikely to be feasible.

\section{Dealing with missing data}

We obtained missing data from study authors, if feasible, and performed intention-to-treat (ITT) analyses whenever possible; otherwise, we performed available case analyses. We investigated attrition rates, e.g. dropouts, losses to follow-up and withdrawals, and appraised any issues of missing data critically.

\section{Assessment of heterogeneity}

We evaluated clinical diversity (clinical heterogeneity) across studies by examining the description of the characteristics of participants, interventions and outcome assessment from data extracted (see Data extraction and management). In the event of significant heterogeneity that could not be explained by subgroup analyses, we did not report outcome results as a pooled effect estimate in a meta-analysis, but provided a narrative description of the results of each study.

We had also planned to identify heterogeneity (inconsistency) through visual inspection of the forest plots to assess the amount of overlap of $\mathrm{Cls}$, and the 12 statistic: this statistic quantifies inconsistency across studies to assess the impact of heterogeneity on the meta-analysis (Higgins 2002; Higgins 2003). We had planned to interpret the $\mathrm{I}^{2}$ statistic as follows:

- $0 \%$ to $40 \%$ : might not be important;

- 30\% to 60\%: may represent moderate heterogeneity;

- $50 \%$ to $90 \%$ : may represent substantial heterogeneity;

- $75 \%$ to $100 \%$ : represents considerable heterogeneity.

When we found moderate (or more) heterogeneity, we had planned to attempt to determine possible reasons for it by examining individual study and subgroup characteristics, as well as by sensitivity analyses according to risk of bias domains.

\section{Assessment of reporting biases}

We attempted to obtain study protocols to assess selective outcome reporting.

If we had included ten studies or more investigating a particular outcome, we planned to use funnel plots to assess small study effects. Several explanations can be offered for the asymmetry of a funnel plot, including true heterogeneity of effect with respect to trial size, poor methodological design (and hence bias of small trials) and publication bias. We therefore interpreted results carefully.

\section{Data synthesis}

Unless there was good evidence for homogeneous effects across studies, we had planned to summarise data using a randomeffects model. We planned to interpret random-effects metaanalyses with due consideration of the whole distribution of effects. In addition, we planned to perform statistical analyses according to the statistical guidelines contained in the Cochrane Handbook for Systematic Reviews of Interventions (Higgins 2011a). For dichotomous outcomes, we planned to use the MantelHaenszel method; for continuous outcomes, we planned to use the inverse variance method; and for time-to-event outcomes, we planned to use the generic inverse variance method to combine final scores or change score of continuous outcomes. We used Review Manager 5 software to perform some analyses (Review Manager 2014).

Since we found incompletely reported outcome data and, in some cases, clinical and methodological diversity, we also performed a narrative synthesis of the available quantitative data following the Cochrane Handbook's guidance (McKenzie 2019) and the latest guidance on Synthesis Without Meta-analysis (SWiM) (Campbell 2020). Firstly, we grouped each comparison by valve type (regardless of manufacturer or opening pressure) following our initial protocol. Then we reported, when available, the absolute number of events for each outcome and the corresponding statistics ( $P$ values). If sufficient data were available, we reported risk ratios or hazard ratios and their $95 \%$ confidence intervals or risk differences when absolute risks in both groups were $<1 \%$. We summarised the results using vote counting based on direction of effect, prioritising the findings from larger studies and, when available, studies at low risk of bias. We assessed heterogeneity qualitatively and followed GRADE methods for assessing the overall quality of evidence and presenting results in 'Summary of findings' tables. 


\section{Subgroup analysis and investigation of heterogeneity}

If we had at least 10 studies, we would have conducted subgroup analyses according to the type of valve employed and also by a number of factors prespecified below, as it is probable that, given the heterogeneity of the causes of hydrocephalus, clinical outcomes may depend on these subgroups:

- type of valve employed: programmable valve systems, gravitational, anti-siphon, flow-regulating and standard valves;

- age of participant: paediatric participants (i.e. from birth to 18 th birthday) and adults;

- congenital or acquired hydrocephalus.

If there were sufficient studies, we used the test for subgroup differences in Review Manager 2014 to compare subgroup analyses.

\section{Sensitivity analysis}

We had planned to perform sensitivity analyses in order to explore the influence of the following factors (when applicable) on effect sizes:

- restricting the analysis by taking into account risk of bias, by excluding studies at high risk or unclear risk, in the following domains: sequence generation (selection bias), allocation concealment (selection bias), blinding of participants and personnel (performance bias), blinding of outcome assessment (detection bias), incomplete outcome data (attrition bias), selective outcome reporting (reporting bias);

- by using the fixed-effect model instead of random-effects model when moderate heterogeneity was present.

\section{Summary of findings and assessment of the certainty of the evidence}

We presented the overall quality of the evidence for each outcome after applying the methods of the GRADE Working Group (GRADE Working Group 2004), which takes into account five criteria that relate to internal validity (risk of bias, inconsistency, imprecision, publication bias), and also to external validity, such as directness of results (Guyatt 2008). For each comparison, independently, two review authors (LIG and JVAF) rated the certainty of evidence for each outcome as 'high', 'moderate', 'low', or 'very low' using GRADEpro 2008. We resolved any discrepancies by consensus, or, if needed, by arbitration by a third review author (AC).
For each comparison, we presented a summary of the evidence for the main outcomes in a 'Summary of findings' table, which provides key information about the best estimate of the magnitude of the effect in relative terms and absolute differences for each relevant comparison of alternative management strategies; numbers of participants and studies addressing each important outcome; and the rating of the overall confidence in effect estimates for each outcome (Guyatt 2011; Schünemann 2011). If meta-analysis was not possible, we presented results in a narrative 'Summary of findings' table.

We created a 'Summary of findings' (SoF) table using the following comparisons:

- Comparison 1: Standard valve compared to anti-syphon valve

- Comparison 2: Comparison between different types of standard valves

- Comparison 3: Standard valve compared to self-adjusting CSF flow-regulating valve

- Comparison 4: External differential programmable valve versus non-programmable valves

The SoF tables included the following outcomes:

- Treatment failure beyond the early postoperative period $>30$ days)

- Adverse events beyond the early postoperative period (> 30 days)

- Mortality in the early postoperative period ( $\leq 30$ days)

- Mortality beyond the early postoperative period (>30 days)

- Quality of life beyond the early postoperative period (> 30 days)

\section{RE S U L T S}

\section{Description of studies}

\section{Results of the search}

For detailed information about the results of the search, see Figure 1. For this review, we screened 2034 records. We excluded 1989 records after title and abstract screening and obtained the full text for 49 studies. We excluded 39 studies after the full-text assessment (their characteristics are described in Characteristics of excluded studies). We included six studies (nine records) in this review. 
Figure 1. Study flow diagram.

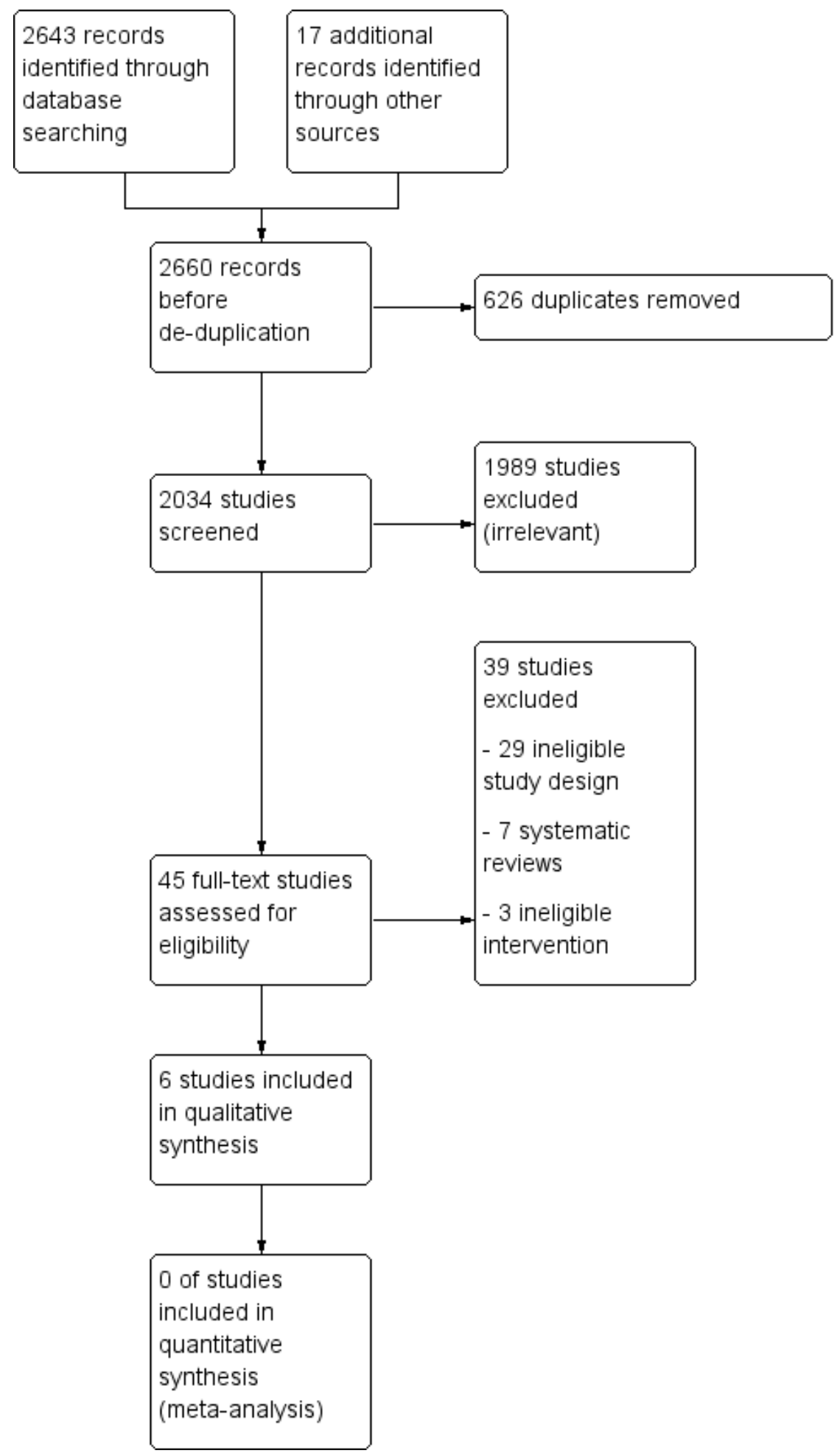

\section{Included studies}

We included six studies with 962 participants (see Characteristics of included studies).

\section{Design}

Four of the included studies were RCTs (Drake 1998; Garcia 1988; Khan 2010; Pollack 1999) and two studies (Liniger 2003; Warf 2005) reported an inadequate method of randomisation (quasirandomised studies). 


\section{Sample sizes}

Median sample size was 87 participants (interquartile range 37 to 352). The smallest sample size was 27 (Liniger 2003) and the largest sample size was 377 (Pollack 1999).

\section{Setting}

Since VP shunt requires a surgical procedure, all included studies started in a hospital setting and offered ambulatory follow-up.

One study was conducted in Mexico (Garcia 1988), one study was conducted in India (Khan 2010), one study was conducted in Switzerland (Liniger 2003), one study was conducted in the USA (Pollack 1999), one study was conducted in Uganda (Warf 2005), and one study was conducted in more than one country (USA, Canada and France; Drake 1998).

Five studies were reported in the English language (Drake 1998; Khan 2010; Liniger 2003; Pollack 1999; Warf 2005), and one study was reported in the Spanish language (Garcia 1988).

\section{Participants}

Four studies were conducted in infants or children (Drake 1998; Khan 2010; Liniger 2003; Warf 2005), one study was conducted in adults (Garcia 1988), and one study was conducted in patients of any age (Pollack 1999).

\section{Interventions}

Two studies compared the placement of a standard valve versus an anti-siphon valve (Khan 2010; Liniger 2003), two studies compared two different types of standard valves (Garcia 1988; Warf 2005), one study compared a programmable valve versus other types of non-programmable valves (Pollack 1999), and one study compared more than two types of valves: a standard valve, an antisiphon valve and a self-adjusting CSF flow-regulating valve (Drake 1998).

\section{Outcomes}

All studies reported the effects of the interventions on treatment failure and adverse events. Three studies reported all-cause mortality (Drake 1998; Garcia 1988; Warf 2005). Three studies reported ventricular size reduction (Khan 2010; Liniger 2003; Pollack 1999), two studies reported head circumference (Khan 2010; Liniger 2003). None of the included studies reported quality of life.

\section{Funding sources}

Most studies (4/6 studies, 67\%) did not specify their funding sources. Drake 1998 was funded by Medtronic PS Medical (Goleta, CA), Cordis Corp (Miami FL), British Columbia Health Research Foundation and The Science Council of British Columbia (Burnaby Canada), Mr. and Mrs. PA Woodward's Foundation (Vancouver Canada) British Columbia's Children's Hospital Telethon Clinical Investigative Initiatives (Vancouver, Canada) British Columbia 's Medical Services Foundation (Vancouver, Canada) Spina Bifida Association of Canada (Winnipeg, Canada) and Spina Bifida Association of British Columbia (Surrey, Canada). Warf 2005 was funded by the International Federation for Spina Bifida and Hydrocephalus and CURE International (which funds CURE Children's Hospital of Uganda).

\section{Excluded studies}

We excluded 39 reports after full-text assessment. Two reports were duplicates that had not been identified in the previous stages (Drake 1996; Drake 2000), one was a letter (Czosnyka 2000), one was an editorial (Hoshide 2017), one was a narrative review (Drake 1998a), nine studies were systematic reviews or health technology assessments (ASERNIP-S 2014; Baird 2014; Del Bigio 1998; Ito 2012; Li 2017; Rasul 2012; Wong 2012; Xu 2013; Xu 2013a), three were in vitro studies (Czosnyka 1990; Czosnyka 2002; Portnoy 1976) and three were randomised trials with ineligible interventions (Mbabazi-Kabachelor 2019; Sinha 2012; Sotelo 2005). The remaining 19 studies were excluded since they were observational studies (mostly retrospective studies or case series).

\section{Risk of bias in included studies}

All studies were deemed to be at unclear or high risk of bias (see Figure 2 and Figure 3)

\section{Figure 2. Risk of bias graph: review authors' judgements about each risk of bias item presented as percentages across all included studies.}

Random sequence generation (selection bias)
Allocation concealment (selection bias)
Blinding of participants and personnel (performance bias): Subjective outcomes: treatment failure, adverse events, quality of life
Blinding of outcome assessment (detection bias): Subjective outcomes: treatment failure, adverse events, quality of life
Blinding of outcome assessment (detection bias): Objective outcomes: mortality, head circumference, ventricular size
Incomplete outcome data (attrition bias): All outcomes
Selective reporting (reporting bias)


Figure 3. Risk of bias summary: review authors' judgements about each risk of bias item for each included study.

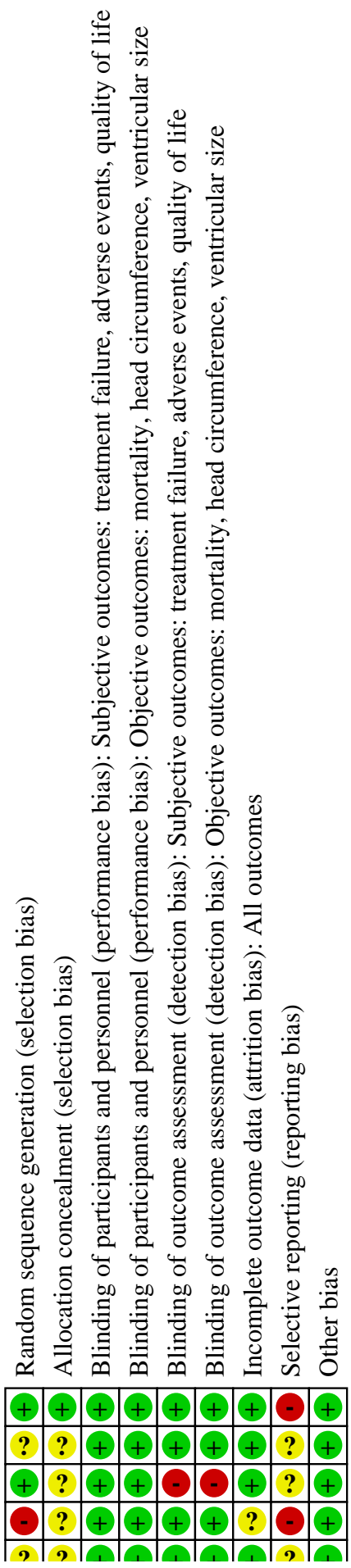


Figure 3. (Continued)

\begin{tabular}{|c|c|c|c|c|c|c|c|c|c|}
\hline Liniger 2003 & & ? & + & + & + & + & $?$ & & + \\
\hline Pollack 1999 & ? & $?$ & + & + & + & + & + & ? & + \\
\hline Warf 2005 & & $?$ & + & + & + & + & - & ? & + \\
\hline
\end{tabular}

\section{Allocation}

\section{All outcomes}

Two studies described an adequate random sequence generation (low risk of bias: Drake 1998; Khan 2010). Two studies provided insufficient information to make a judgement (unclear risk of bias: Garcia 1988; Pollack 1999) and two studies performed inadequate methods for randomisation or included a nonrandomised component for participants' allocation (high risk of bias: Liniger 2003; Warf 2005). Only one study adequately described concealment of allocation (low risk of bias: Drake 1998), while the rest of the studies provided insufficient information to make a judgement (unclear risk of bias).

\section{Blinding}

Subjective outcomes: treatment failure, adverse events, quality of life

Considering that the interventions were visibly different between groups, none of the studies were blinded (high risk of performance bias). However, one study had an independent assessment of outcomes by a blind adjudication committee (low risk of detection bias: Drake 1998).

\section{Objective outcomes: mortality, head circumference, ventricular size}

We judged separately objective outcomes, for which we considered these studies to be at a low risk of performance and detection bias.

\section{Incomplete outcome data}

\section{All outcomes}

Outcome data was available for all participants in four studies (low risk of bias: Drake 1998; Garcia 1988; Khan 2010; Pollack 1999). There was no information on missing outcome data in one study (unclear risk of bias: Liniger 2003). One study excluded participants that were lost to follow-up from the analysis and there was an unbalanced attrition (high risk of bias: Warf 2005).

\section{Selective reporting}

\section{All outcomes}

Risk of bias was unclear for most studies as there were no study protocols (Garcia 1988; Khan 2010; Pollack 1999; Warf 2005). However, two studies failed to report predefined outcomes or time points and presented outcome data (e.g. mortality) globally but not by each treatment group (Drake 1998; Liniger 2003); these studies were judged as being at high risk of bias.

\section{Other potential sources of bias}

\section{All outcomes}

No other sources of bias were detected.

\section{Effects of interventions}

See: Summary of findings 1 Standard valve compared to anti-syphon valve for hydrocephalus; Summary of findings 2 Comparison between different types of standard valves; Summary of findings 3 Standard valve compared to self-adjusting CSF flow-regulating valve for hydrocephalus; Summary of findings 4 External differential programmable pressure valve compared to non-programmable valves

\section{Standard valve versus anti-siphon valve}

Three studies with 296 randomised participants were included under this comparison (Drake 1998; Khan 2010; Liniger 2003). One study compared standard valves versus a group of valves including the anti-siphon valve for which no disaggregated data was available, therefore this study is described separately (Pollack 1999). Drake 1998 compared a standard valve versus a Delta antisiphon valve; Khan 2010 compared a group of standard valves (Chabbra and Cederain) versus a Vygon anti-siphon valve; Liniger 2003 compared a standard (Pudenz mid-pressure) valve versus a Delta anti-syphon valve. See Summary of findings 1.

\subsection{Treatment failure}

We are uncertain about the incidence of treatment failure in participants with standard valve compared to anti-siphon valves:

- Drake 1998 reported this outcome in 229 participants. The authors stated that "there were no differences among the three shunt valve groups, either by log-rank tests for equality of the three curves (log-rank $=2.90, \mathrm{P}=0.24$ )" [the third arm included a Orbis-Sigma valve]. The incidence of treatment failure at two years follow-up was: $42.98 \%$ in the standard valve group (39 shunt obstruction, 3 over-drainage, 7 shunt infection) and $48.69 \%$ in the Delta anti-syphon valve group (38 shunt obstruction, 9 over-drainage, 9 shunt infection).

- Khan 2010 reported this outcome in 40 participants. The incidence of treatment failure at three to six months follow-up included: four cases of shunt obstruction and four cases of shunt infection and no cases of over-drainage with the Vygon antisiphon valve; and three cases of shunt obstruction, three cases of shunt infection and two cases of shunt over-drainage with the standard valve (reported $P$ values were $1.00,1.00$ and 0.49 respectively for these comparisons).

- Liniger 2003 reported this outcome in 27 participants. The incidence of emergency treatment failure at nine years of clinical follow-up included six (55\%) of 11 participants in the group of shunt revision with the standard and eight (50\%) of 16 participants in the group of shunt revision with the Delta antisiphon valve. When elective revisions were included in the analysis, $63 \%$ of participants suffered treatment failure (no disaggregated data by each type of intervention was available). In the early follow-up (six months from shunt insertion), 30\% of participants with standard valves and $14 \%$ with anti-siphon 
valves developed a "slit ventricle" ( $n=24$ participants) and $64 \%$ of participants with standard valves and $38 \%$ with antisyphon valves developed slit ventricle in the late follow-up (at six years of age, $n=27$ participants). The authors also reported that $9 \%$ of participants with standard valves and $6.25 \%$ of participants with anti-syphon valves developed a "slit ventricle syndrome", defined as slit ventricles in radiological studies with recurrent clinical signs of intracranial hypertension, namely severe headaches and emesis, and slow or no refill of the valve.

The certainty of the evidence is very low due to concerns about bias (downgraded two levels due to high or unclear risk of bias in multiple domains of the included studies) and imprecision (downgraded one level due to few participants and events in each study).

\subsection{Adverse events}

The incidence of adverse events was reported by one study with 229 participants (Drake 1998). The incidence of adverse events may be similar in those with standard valves and antisiphon valves. With the Delta anti-siphon valve, $0 \%$ of participants developed an intracranial haemorrhage, $0 \%$ developed a new or increased neurological deficit, $0 \%$ developed a neck injury, $0.9 \%$ developed a chest injury, $0 \%$ developed an abdominal injury, $0.9 \%$ developed an inadvertent skin perforation along the shunt tract, $1.7 \%$ developed a wound dehiscence, $2.9 \%$ developed a postoperative subcutaneous fluid collection, and $0 \%$ developed a transient wound cerebrospinal fluid leak, while with the standard valve $0.9 \%$ of participants developed an intracranial haemorrhage, $0 \%$ developed a new or increased neurological deficit, $0.9 \%$ developed a neck injury, $0.9 \%$ developed a chest injury, $0 \%$ developed an abdominal injury, $0 \%$ developed an inadvertent skin perforation along the shunt tract, $1.8 \%$ developed a wound dehiscence, $1.9 \%$ developed a postoperative subcutaneous fluid collection, and $0.9 \%$ developed a transient wound cerebrospinal fluid leak.

The other studies did not report adverse events beyond those reported under "treatment failure" (Khan 2010; Liniger 2003).

The certainty of the evidence is low due to concerns about bias and imprecision.

\subsection{Mortality}

Only one study with 229 participants reported this outcome under this comparison (Drake 1998). Mortality may be similar in those with standard valves and anti-siphon valves.

In the postoperative period, $0.9 \%$ of participants died with the Delta anti-siphon valve. No participant with the standard valve died in this period (risk difference [RD] $0.01 \%, 95 \% \mathrm{Cl}-0.02 \%$ to $0.03 \%$, Analysis 1.1).

Beyond the early postoperative period, the authors stated that "nine patients died, four as a result of a progression of neoplastic disease and the others as a result of hyponatraemia, sepsis, progressive respiratory disease, gastroenteritis, or sudden infant death", but no disaggregated data by group was available.

The certainty of the evidence is low due to concerns about bias and imprecision.

\subsection{Quality of life}

None of the included studies reported this outcome.

\subsection{Ventricular size reduction}

Ventricular size may be similar in those with standard valves and anti-syphon valves.

Khan 2010 reported the Modified Evan's index (MEI) at six-month follow-up and stated "the decrease in mean MEl from 0 month to three months was more in the non-anti-siphon group than the anti-siphon group but it was statistically not significant" (data were presented graphically, P values not available) (Khan 2010).

Liniger 2003 reported that ventricular surface measured at six years follow-up was similar with both valves: standard valve $8.5 \mathrm{~cm}^{2}$ (95\% $\mathrm{Cl}, 4.2-12,7)$ and anti-siphon valve $5.8 \mathrm{~cm}^{2}(95 \% \mathrm{Cl}, 4.0-7.5) \mathrm{P}$ value $=0.45$.

The certainty of the evidence is low due to concerns about bias and imprecision.

\subsection{Head circumference}

Two studies with 67 participants reported this outcome (Khan 2010; Liniger 2003). Head circumference may be similar in those with standard valves and those with anti-syphon valves. In one study at six months follow-up, the mean occipital head circumference was $35.86 \mathrm{~cm}$ with the standard valve and $36.6 \mathrm{~cm}$ with the anti-siphon valve (no standard deviation data were available) (Khan 2010). In the other study, the median (range) head circumference at a corrected age of 7 years was $51 \mathrm{~cm}(48-56)$ with the standard valve and $52 \mathrm{~cm}$ (46-55) with the anti-siphon valve (Liniger 2003).

The certainty of the evidence is low due to concerns about bias and imprecision.

\section{Comparison between different types of standard valves}

Two studies with 174 randomised participants were included under this comparison (Garcia 1988; Warf 2005). Garcia 1988 compared a standard Hakim ball-in-cone and spring valve versus a standard distal cleft valve; and Warf 2005 compared a standard Chhabra valve versus a standard Codman-Hakim micro precision valve. See Summary of findings 2.

\subsection{Treatment failure}

We are uncertain about the incidence of treatment failure in participants with different types of standard valves:

- Garcia 1988 reported this outcome in 84 participants. A total of 26 treatment failures occurred $(30.9 \%$ global incidence of treatment failure), four with the standard distal cleft valve and eight with the standard Hakim ball-in-cone and spring valve during the early postoperative period (30 days), while five participants with the standard distal cleft valve and nine with the standard Hakim ball-in-cone and spring valve developed treatment failure during the late follow-up period (early postoperative period: RR $0.41,95 \% \mathrm{Cl} 0.13$ to 1.27 , Analysis 2.1). Treatment failure with the standard distal cleft valve was due to ventricular obstruction in $22 \%$ of participants $(n=2)$, peritoneal obstruction in $56 \%$ of participants ( $n=$ $5)$, hyperfunction in $0 \%$ of participants $(n=0)$, inadequate pressure in $11 \%$ of participants $(n=1)$ and not determined in 
$11 \%$ of participants $(n=1)$. With the standard Hakim ball-incone and spring valve, treatment failure was due to ventricular obstruction in $18 \%$ of participants $(n=3)$, peritoneal obstruction in $24 \%$ of participants $(n=4)$, hyperfunction in $12 \%$ of participants $(n=2)$, inadequate pressure in $0 \%$ of participants ( $n$ $=0)$, and not determined in $46 \%$ of participants $(n=8)$.

- Warf 2005 reported this outcome in 90 participants. At 12 months follow-up, 21 participants with the Chabbra valve and 15 participants with the Codman-Hakim micro precision valve suffered complications (RR $1.17,95 \% \mathrm{Cl} 0.72$ to 1.92 , Analysis 2.1). Seven percent $(n=3)$ of participants with the Chhabra valve developed valve malfunction, 9.3\% ( $\mathrm{n}=$ 4) developed infections, $2.3 \%(n=1)$ developed proximal obstruction, 2.3\% $(n=1)$ developed distal obstruction and 9.7\% $(n=3)$ developed migration. With the Codman-Hakim micro precision valve, $0 \%$ of participants $(n=0)$ developed valve malfunction, $11 \%(n=4)$ developed infections, $2.7 \%(n=1)$ developed proximal obstruction, $0 \%(n=0)$ developed distal obstruction and $8.3 \%(n=3)$ developed migration.

The certainty of the evidence is very low due to concerns about bias (downgraded two levels due to high or unclear risk of bias in multiple domains of the included studies) and imprecision (downgraded one level due to few participants and events in each study).

\subsection{Adverse events}

None of the included studies reported this outcome beyond those included under "treatment failure".

\subsection{Mortality}

We are uncertain about the effects of different types of standard valves on mortality:

- Warf 2005 reported that in the early postoperative period (onemonth follow-up), $2 \%(n=1)$ participant died with the Chhabra valve, while no participant with the Codman-Hakim micro precision valve died in this period (RD $0.02 \%, 95 \% \mathrm{Cl}-0.04 \%$ to $0.09 \%$, Analysis 2.2 ). Beyond the early postoperative period (12 months follow-up), $14 \%(n=6)$ of participants died with the Chhabra valve while $17 \%(n=6)$ of participants died with the Codman-Hakim micro precision valve (RD $-0.03 \%, 95 \% \mathrm{Cl}-0.19 \%$ to $0.13 \%$, Analysis 2.2 ).

- Garcia 1988 reported that four participants with the standard distal cleft valve and no participant with the standard Hakim ball-in-cone and spring valve died in the early postoperative period (RD $0.09 \%, 95 \% \mathrm{Cl}-0.00 \%$ to $0.18 \%$, Analysis 2.2 ). The cause of death with the first valve was pneumonia and sepsis (two cases), ependymitis (one case) and brain stem compression (one case).

The certainty of evidence is very low due to concerns about risk of bias and imprecision (few events).

None of the included studies reported the effects of these interventions on quality of life, ventricular size reduction and head circumference.

\section{Standard valve versus self-adjusting CSF flow- regulating valve}

One study with 229 randomised participants addressed this comparison (Drake 1998). This study compared a standard valve with the Orbis-Sigma self-adjusting CSF flow-regulating valve (Cordis). See Summary of findings 3.

\subsection{Treatment failure}

Drake 1998 reported this outcome in 229 participants. The incidence of treatment failure may be similar in those with the standard valve and the constant flow valve. The incidence of treatment failure at two years follow-up was $42.98 \%$ with the standard valve (39 shunt obstruction, 3 over-drainage, 7 shunt infection, 0 loculated compartments) and $39.13 \%$ with the OrbisSigma valve (31 shunt obstruction, 0 over-drainage, 12 shunt infection, 2 loculated compartments) (RR 1.08, 95\% Cl 0.79 to 1.47 , Analysis 3.1).

The certainty of the evidence is low due to concerns about bias (high or unclear risk of bias in multiple domains of the included study) and imprecision (few events leading to a wide confidence interval).

\subsection{Adverse events}

Drake 1998 reported postoperative complications. The incidence of adverse events may be similar in those with the standard valve and those with the self-adjusting CSF flow-regulating valve. With the standard valve, $0.9 \%$ of participants developed intracranial haemorrhage, $0 \%$ developed new or increased neurological deficit, $0.9 \%$ developed neck injury, $0.9 \%$ developed chest injury, $0 \%$ developed abdominal injury, $0 \%$ developed inadvertent skin perforation along shunt tract, $1.8 \%$ developed wound dehiscence, $1.9 \%$ developed postoperative subcutaneous fluid collection and $0.9 \%$ developed transient wound cerebrospinal fluid leak; with the Orbis-Sigma self-adjusting CSF flow-regulating valve, $0 \%$ of participants developed intracranial haemorrhage, $0 \%$ developed new or increased neurological deficit, $0 \%$ developed neck injury, $0.9 \%$ developed chest injury, $0 \%$ developed abdominal injury, $0 \%$ developed inadvertent skin perforation along shunt tract, $0 \%$ developed wound dehiscence, $7.2 \%$ developed postoperative subcutaneous fluid collection and $0.9 \%$ developed transient wound cerebrospinal fluid leak.

The certainty of the evidence is low due to concerns about bias (high or unclear risk of bias in multiple domains of the included study) and imprecision.

\subsection{Mortality}

Drake 1998 reported no deaths with either valve in the postoperative period. Beyond the early postoperative period, the authors stated that "nine patients died, four as a result of progression of neoplastic disease and the others as a result of hyponatraemia, sepsis, progressive respiratory disease, gastroenteritis, or sudden infant death", but no disaggregated data by each type of intervention were available.

The certainty of the evidence is low due to concerns about bias and imprecision. 
None of the included studies reported the effects of these interventions on quality of life, ventricular size reduction and head circumference.

\section{External differential programmable pressure valve versus non-programmable valve}

One study with 377 randomised participants addressed this comparison (Pollack 1999). The study compared a Codman-Hakim programmable valve system versus any other non-programmable fixed pressure valves of surgeon's choice available on the market (including Delta anti-syphon valves, Orbis-Sigma valves and other Medtronic PS Medical or other Codman/Johnson \& Johnson valves) with no disaggregated data available within this group. See Summary of findings 4.

\subsection{Treatment failure}

Pollack 1999 reported this outcome in 377 participants. The incidence of treatment failure may be similar in those with the programmable valve and the other non-programmable types of valves. In the two years' follow-up, $52 \%(n=100)$ of participants with the programmable valve and $52 \%(n=96)$ of participants with the other non-programmable fixed pressure valves developed treatment failure (RR $1.02,95 \% \mathrm{Cl} 0.84$ to 1.24 , Analysis 4.1 ). With the programmable valve, treatment failure occurred in $52 \%$ (n $=62$ ) of 119 participants who underwent initial shunt insertion and in $43 \%(n=32)$ of 75 participants who underwent an existing valve replacement. With the other non-programmable fixed pressure valves, treatment failure occurred in $50 \%(n=58)$ of 116 participants who underwent initial shunt insertion and in $43 \%(n=29)$ of 67 participants who underwent an existing valve replacement. Infections requiring explantation was developed in $10.8 \%(n=21)$ of participants with the programmable valve and in $8.7 \%(n=16)$ participants with the other non-programmable fixed pressure valves.

The certainty of the evidence is moderate due to concerns about bias.

\subsection{Adverse events}

The incidence of adverse events may be similar in those with the programmable valve and the other non-programmable types of valves.

Pollack 1999 reported subdural haematomas or hygromas in $6.19 \%$ $(n=12)$ of participants with the programmable valve and in $6.01 \%(n=11)$ of participants with the other non-programmable fixed pressure valves (RR $0.97,95 \% \mathrm{Cl} 0.44$ to 2.15 , Analysis 4.2). The authors stated that "the majority of other complications assessed as a part of the study (haemorrhage, malabsorption, catheter perforation, etc.) were extremely uncommon and were reported in $1 \%$ or less of either the experimental or control groups."

The certainty of the evidence is low due to concerns about bias and imprecision.

Ventricular size reduction may be similar in those with the programmable valve and the other non-programmable types of valves (low certainty of evidence). The included study did not report the effects of these interventions on mortality, quality of life and head circumference.

\section{DISCUSSION}

\section{Summary of main results}

We included six studies with 962 participants assessing the effects of standard valves compared to anti-siphon valves, other types of standard valves, self-adjusting CSF flow-regulating valves and external differential programmable pressure valves versus nonprogrammable valves. The certainty of the evidence for most comparisons was low to very low.

\section{Standard valve versus anti-siphon valve}

Three studies with 296 randomised participants were included under this comparison. We are uncertain about the incidence of treatment failure in participants with standard valve and anti-siphon valves (very low certainty of the evidence). The incidence of adverse events may be similar in those with standard valves and anti-siphon valves (low certainty of the evidence). Mortality may be similar in those with standard valves and antisiphon valves (low certainty of the evidence). Ventricular size and head circumference may be similar in those with standard valves and anti-siphon valves (low certainty of the evidence). None of the included studies reported on the quality of life of participants.

\section{Comparison between different types of standard valves}

Two studies with 174 randomised participants were included under this comparison We are uncertain about the incidence of treatment failure in participants with different types of standard valves (very low certainty of the evidence). None of the included studies reported adverse events beyond those included under "treatment failure". We are uncertain about the effects of different types of standard valves on mortality (very low certainty of the evidence). The included studies did not report the effects of these interventions on quality of life, ventricular size reduction or head circumference.

\section{Standard valve versus self-adjusting CSF flow-regulating valve}

One study with 229 randomised participants addressed this comparison. The incidence of treatment failure may be similar in those with the standard valve and the self-adjusting CSF flowregulating valve (low certainty of the evidence). The incidence of adverse events may be similar in those with the standard valve and those with the self-adjusting CSF flow-regulating valve (low certainty of the evidence). The included study reported no deaths in either group in the postoperative period. Beyond the early postoperative period, the authors stated that nine patients died (no disaggregated data for each type of intervention was available, low certainty of the evidence). The included studies did not report the effects of these interventions on quality of life, ventricular size reduction or head circumference.

\section{External differential programmable pressure valve versus non-programmable valve}

One study with 377 randomised participants addressed this comparison. The incidence of treatment failure may be similar in those with programmable valves and the other types of nonprogrammable valves (low certainty of the evidence). The incidence of adverse events may be similar in those with programmable valves and the other types of non-programmable valves (low 
certainty of the evidence). The included study did not report the effect of these interventions on mortality, quality of life or head circumference. Ventricular size reduction may be similar in those with programmable valves and the other types of nonprogrammable valves (low certainty of the evidence).

\section{Overall completeness and applicability of evidence}

Participants with the most frequent types of hydrocephalus were included across the different included studies. These were conducted mainly in children and infants, similar to those that can be found in clinical practice. Only one study was conducted in adults, so this population might not be well represented.

We did not find studies assessing some of the planned interventions. The use of more advanced technology in valves, such as those with anti-gravitational mechanisms plus the possibility of external regulation, have yet to be tested versus those of fixed pressure or anti-siphon devices. Furthermore, current interventions for hydrocephalus might be considered different from those in the included studies, due to the emergence of more complex devices with anti-siphon systems combined with different degrees of fixed pressures or external regulation. Additionally, when the included studies were conducted, endoscopic thirdventriculostomy was not available for the treatment of hydrocephalus, probably because of the lack of training among neurosurgeons and the lack of endoscopy instruments, making this intervention not available worldwide. Nowadays, it has become a viable option when valves are not available or when the etiology of hydrocephalus is associated with higher success with this procedure.

Not all the outcomes of interest for this review have been reported in the included studies. We also identified heterogeneity in outcome definition among studies. In some studies, valve setting was modified by each surgeon's discretion and patient outcomes related to those modifications (i.e. ventricle size) were not independently reported, not allowing a proper assessment. However, the measurement of the outcomes nowadays remains similar, with a similar degree of subjectivity. Nevertheless, by recent improvements of imaging, ultrasound, CT scan and MRI, there is a possibility of taking uniform and more objective measures that could be used to establish uniform criteria on outcome definition. Similarily, neuropsychological studies have been developed recently and can adequately estimate the quality of life of patients treated for hydrocephalus. It is important to highlight that some adverse events may be more dependent on the shunting procedure than the type of device itself (e.g. slit ventricle, subdural collection, infection, etc.).

\section{Quality of the evidence}

The overall quality of the evidence was low to very low. All studies had high risk of bias, mainly due to lack of blinding leading to possible performance bias influencing the main outcomes. Even for outcomes that might be unaffected by blinding, we found concerns regarding the randomisation and allocation process, missing data and selective reporting. Some studies were very small and had few events that led to important imprecision. We were unable to assess publication bias due to the scarcity of studies per outcome.

\section{Potential biases in the review process}

We rearranged and redefined the outcomes to maximise the use of available data. We took precautions to avoid bias in this process by documenting all changes in the section Differences between protocol and review. We also initially planned to conduct meta-analysis but the quality of the reports was so low and sparse that we opted for Synthesis Without Meta-analysis (SWiM), which is a new method available in the Cochrane Handbook. We were unable to conduct prespecified sensitivity and subgroup analyses. We attempted to minimise publication bias in the review by searching multiple databases including trial registers and conference proceedings.

\section{Agreements and disagreements with other studies or reviews}

We identified nine systematic reviews or health technology assessments. Four reviews did not provide comparative effectiveness data: three reviews included only descriptive data from large case series (Ito 2012) or a mix of randomised trials and observational studies (Wong 2012), one health technology assessment (ASERNIP-S 2014) provided descriptive data from case series of a gravitational valve, and one review (Del Bigio 1998) summarised data from in vitro studies.

One review (Baird 2014) incorporated most of the studies in our review and a series of observational studies. This review found a similar incidence of shunt failure across different types of valves including programmable valves and anti-siphon valves. While some observational studies indicated that programmable valves (Strata and Codman-Hakim) may have a greater incidence of shunt failure when compared to other valves, these results were highly inconsistent (Baird 2014).

Another review (Li 2017) included three randomised studies and eight observational studies. Two randomised studies included patients with normal pressure hydrocephalus. This review found no difference in mortality or complications in programmable valves versus non-programmable valves. Based on data from observational studies at high risk of bias, a reduced incidence of shunt revision rates was observed with programmable valves. Another review found similar results (Rasul 2012).

A review team published two systematic reviews (Xu 2013; Xu 2013a). These reviews included randomised controlled trials and observational studies that were pooled in meta-analysis and indicated that there were fewer complications and revision rates with programmable valves when compared to non-programmable valves. However, the results from the two versions of the review differed substantially. Furthermore, these results were primarily driven by observational studies.

This summary suggests that a few reviews found different results from ours, but this was mostly driven by observational studies. Furthermore, none of the mentioned reviews were of high quality and none of them incorporated GRADE methods in the assessment of the certainty of the evidence. 


\section{AUTHORS' CONCLUSIONS}

\section{Implications for practice}

Standard shunt valves for hydrocephalus compared to anti-siphon or self-adjusting CSF flow-regulating valves may cause little to no difference on the main outcomes of this review; however, we are very uncertain due to the low to very low quality of evidence. Similarly, different types of standard valves and programmable versus non-programmable valves may be associated with similar outcomes. Nevertheless, this review did not include valves with the latest technology.

\section{Implications for research}

High-powered RCTs are needed, especially those that consider the latest technology including those that combine different degrees of fixed pressures or those with externally-regulated pressure with the addition of anti-siphon or antigravitational devices. These studies should consider major outcomes for all stakeholders like patients, their families, physicians, surgeons, funders and policy-makers. The cost associated with the procedure, both direct and indirect, should also be considered. There is also a need for researchers to establish standardised outcome definitions and timings for their measurements, including newer, more objective and reliable assessment methods.

\section{ACKNOWLEDGEMENTS}

The Cochrane Multiple Sclerosis and Rare Diseases of the Central Nervous System Review Group, Carlo Besta Neurological Institute, Milan, Italy.

We specially want to thank Dr Iván Solá and the Iberoamerican Cochrane Center, for the training and support given to Virginia Garrote for the development of the search strategy. We thank Dr Elizabeth Royle and Dr Katarina Ivana Tudor for useful comments during the preparation of the protocol.

We thank Dr. Jessica Cail for assisting us with the provision of fulltext articles identified in our search. 


\section{RE F E R E N C E S}

\section{References to studies included in this review}

\section{Drake 1998 \{published data only\}}

Drake JM, Kestle J, Boop F, Cochrane D, Haines S, Sainte-Rose C, et al. Rationale and methodology of the multicenter pediatric cerebrospinal fluid shunt design trial. Child's Nervous System 1996; 12(8):434-47.

Drake JM, Kestle J. Determining the best cerebrospinal fluid shunt valve design: the pediatric valve design trial. Neurosurgery 1996; 38(3):604-7.

* Drake JM, Kestle JR, Milner R, Cinalli G, Boop F, Piatt J, et al. Randomized trial of cerebrospinal fluid shunt valve design in pediatric hydrocephalus. Neurosurgery 1998; 43(2):294-303; discussion 303

Kestle J, Drake J, Milner R, Sainte-Rose C, Cinalli G, Boop F, et al. Long-term follow-up data from the Shunt Design Trial. Pediatric Neurosurgery 2000; 33(5):230-6.

Kestle J, Milner R, Drake D. An assessment of observer bias in the Shunt Design trial. Pediatric Neurosurgery 1999; 30(2):57-61.

Kestle J, Milner R, Drake J. The Shunt Design trial: variation in surgical experience did not influence shunt survival. Pediatric Neurosurgery 1999; 30(6):283-7.

\section{Garcia 1988 \{published data only\}}

Garcia de Llano VCA, Mateos GJH, Kleriga GE. Estudio comparativo de dos sistemas para la derivacion del liquido cefalorraquideo en hidrocefalea. Revista Médica del Instituto Mexicano del Seguro Social 1988; 26(3/4):199-204.

\section{Khan 2010 \{published data only\}}

Khan RA, Narasimhan KL, Tewari MK, Saxena AK. Role of shunts with antisiphon device in treatment of pediatric hydrocephalus. Clinical Neurology and Neurosurgery 2010; 112(8):687-90.

\section{Liniger 2003 \{published data only\}}

Kaiser GL, Horner E, Marchand S, Jost A. Conventional versus Delta valve in the treatment of hydrocephalus in early infancy. European Journal of Pediatric Surgery 1997; 7 Suppl 1:45-6. [PMID: 9497119]

* Liniger P, Marchand S, Kaiser GL. Flow control versus antisiphon valves: late results concerning slit ventricles and slitventricle syndrome. European Journal of Pediatric Surgery 2003; 13 Suppl 1:S3-6.

\section{Pollack 1999 \{published data only\}}

Carmel PW, Albright AL, Adelson PD, Canady A, Black P, Boydston $W$, et al. Incidence and management of subdural hematoma/hygroma with variable- and fixed-pressure differential valves: a randomized, controlled study of programmable compared with conventional valves. Neurosurgical Focus 1999; 7(4):e7.

* Pollack IF, Albright AL, Adelson PD. A randomized, controlled study of a programmable shunt valve versus a conventional valve for patients with hydrocephalus. Neurosurgery 1999; 45(6):1399-411.

\section{Warf 2005 \{published data only\}}

Warf BC. Comparison of 1-year outcomes for the Chhabra and Codman-Hakim Micro Precision shunt systems in Uganda: a prospective study in 195 children. Journal of Neurosurgery 2005; 102(Suppl 4):358-62.

\section{References to studies excluded from this review}

Agarwal 2018 \{published data only\}

Agarwal N, Kashkoush A, McDowell MM, Lariviere WR, Ismail N, Friedlander RM. Comparative durability and costs analysis of ventricular shunts. Journal of Neurosurgery 2018; 130(5):1-8.

ASERNIP-S 2014 \{published data only\}

. Miethke proSA ${ }^{\circledR}$ adjustable gravitational shunt for hydrocephalus (structured abstract). Health Technology Assessment Database 2014.

Baird 2014 \{published data only\}

Baird LC, Mazzola CA, Auguste KI, Klimo P, Flannery AM. Pediatric hydrocephalus: systematic literature review and evidence-based guidelines. Part 5: Effect of valve type on cerebrospinal fluid shunt efficacy. Journal of Neurosurgery: Pediatrics 2014; 14:35-43.

\section{Beez 2014 \{published data only\}}

Beez T, Sarikaya-Seiwert S, Bellstadt L, Muhmer M, . Role of ventriculoperitoneal shunt valve design in the treatment of pediatric hydrocephalus - a single center study of valve performance in the clinical setting. Child's Nervous System 2014; 30(2):293-7.

\section{Czosnyka 1990 \{published data only\}}

Czosnyka M, Maksymowicz W, Batorski L, Koszewski W, Czosnyka Z. Comparison between classic-differential and automatic shunt functioning on the basis of infusion tests. Acto Neurochirurgica 1990; 106(1-2):1-8.

\section{Czosnyka 2000 \{published data only\}}

Czosnyka Z, Czosnyka M, Copeman J, Pickard JD. A randomized, controlled study of a programmable shunt valve versus a conventional valve for patients with hydrocephalus (multiple letters). Neurosurgery 2000; 47(5):1250-1.

\section{Czosnyka 2002 \{published data only\}}

Czosnyka ZH, Czosnyka M, Pickard JD. Shunt testing in-vivo: a method based on the data from the UK shunt evaluation laboratory. Acta Neurochirurgica 2002; 81(Suppl):27-30.

\section{Decq 1995 \{published data only\}}

Decq P, Barat JL, Duplessis E, Leguerinel C, Gendrault P, Keravel Y. Shunt failure in adult hydrocephalus: flow-controlled shunt versus differential pressure shunts - a cooperative study in 289 patients. Surgical Neurology 1995; 43(4):333-9. 
Del Bigio 1998 \{published data only\}

Del Bigio MR. Biological reactions to cerebrospinal fluid shunt devices: a review of the cellular pathology. Neurosurgery 1998; 42(2):319-26

\section{Drake 1996 \{published data only\}}

Drake JM, Kestle J: Pediatric Hydrocephalus Treatment Evaluation Group. Rationale and methodology of the multicenter pediatric cerebrospinal fluid shunt design trial. Child's Nervous System 1996; 12(8):434-47.

\section{Drake 1998a \{published data only\}}

Drake JM, Kestle JT. Determining the best cerebrospinal fluid shunt valve design: the pediatric Valve Design trial. Neurosurgery 1998; 43(5):1259-60.

Drake 2000 \{published data only\}

Drake JM, Kestle JR, Tuli S. CSF shunts 50 years on past, present and future. Child's Nervous System 2000; 16(10-11):800-4.

\section{Eymann 2007 \{published data only\}}

Eymann R, Steudel WI, Kiefer M. Pediatric gravitational shunts: initial results from a prospective study. Journal of Neurosurgery 2007; 106(3 Suppl):179-84.

\section{Felix 1983 \{published data only\}}

Felix Estrada G, Loyo Varela M, Mateos Gomez JH, Leo R, Kleriga E. Nuevo sistema valvular de Felix. Regulador de la presion intracraneal para el control de hidrocefalia. Cirugia y Cirujanos 1983; 51(6):473-7.

\section{Gruber 1984 \{published data only\}}

Gruber R, Jenny P, Herzog B. Experiences with the anti-siphon device (ASD) in shunt therapy of pediatric hydrocephalus. Journal of Neurosurgery 1984; 61(1):156-62.

\section{Haberl 2009 \{published data only\}}

Haberl EJ, Messing-Juenger M, Schuhmann M, Eymann R, Cedzich C, Fritsch MJ, et al. Experiences with a gravity-assisted valve in hydrocephalic children. Journal of Neurosurgery (Pediatrics) 2009; 4(3):289-94.

\section{Higashi 1994 \{published data only\}}

Higashi S, Futami K, Matsuda H, Yamashita J, Hashimoto M, Hasegawa $M$, et al. Effects of head elevation on intracranial hemodynamics in patients with ventriculoperitoneal shunts. Journal of Neurosurgery 1994; 81(6):829-36.

\section{Hoshide 2017 \{published data only\}}

Hoshide R, Meltzer H, Dalle-Ore C, Gonda D, Guillaume D, Chen C. Impact of ventricular-peritoneal shunt valve design on clinical outcome of pediatric patients with hydrocephalus: lessons learned from randomized controlled trials. Surgical Neurology International 2017; 8(1):49.

\section{Ito 2012 \{published data only\}}

Ito M, Houkin K, Saito H, Shimbo D, Motegi H, Kawabori M, et al. Systematic review of complications for proper informed consent: (4) cerebrospinal fluid shunts for hydrocephalus and related disorders. Journal of Neurological Surgery 2012 40(10):923-45.

Jain 2005 \{published data only\}

Jain H, Natarajan K, Sgouros S. Influence of the shunt type in the difference in reduction of volume between the two lateral ventricles in shunted hydrocephalic children. Child's Nervous System 2005; 21(7):552-8.

Kiefer 2000 \{published data only\}

Kiefer M, Eymann R, Mascaros V, Walter M, Steudel WI. Significance of hydrostatic valves in therapy of chronic hydrocephalus [Der Stellenwert Hydrostatischer ventile in der therapie des chronischen hydrozephalus]. Der Nervenarzt 2000; 71(12):975-86.

Legat 1996 \{published data only\}

Legat JA, Eder HG, Oberbauer RW. Over-drainage by hydrocephalus: comparative study of Orbis Sigma and Delta shunt systems. Zentralblatt fur Neurochirurgie 1996; 57 Suppl:9.

\section{Li 2017 \{published data only\}}

Li M, Wang H, Ouyang Y, Yin M, Yin X. Efficacy and safety of programmable shunt valves for hydrocephalus: a meta-analysis. International Journal of Surgery (London, England) 2017; 44:139-46.

Lund-Johansen 1994 \{published data only\}

Lund-Johansen M, Svendsen F, Wester K. Shunt failures and complications in adults as related to shunt type, diagnosis, and the experience of the surgeon. Neurosurgery 1994; 35(5):839-44.

Mbabazi-Kabachelor 2019 \{published data only\}

Mbabazi-Kabachelor E, Shah M, Vaughan KA, Mugamba J, Ssenyonga P, Onen J, et al. Infection risk for Bactiseal Universal shunts versus Chhabra shunts in Ugandan infants: a randomized controlled trial. Journal of Neurosurgery (Pediatrics) 2019; 23(3):397-406

\section{Meling 2005 \{published data only\}}

Meling TR, Egge A, Due-Tonnessen B. The gravity-assisted PaediGav valve in the treatment of pediatric hydrocephalus. Pediatric Neurosurgery 2005; 41(1):8-14.

Portnoy 1976 \{published data only\}

Portnoy HD, Tripp L, Croissant PD. Hydrodynamics of shunt valves. Child's Brain 1976; 2(4):242-56.

\section{Rasul 2012 \{published data only\}}

Rasul FT, Marcus HJ, Toma AK, Thorne L, Watkins LD. In patients with hydrocephalus treated with shunts, are programmable valves superior to nonprogrammable valves? British Journal of Neurosurgery 2012; 26(5):617.

\section{Sainte-Rose 1993 \{published data only\}}

Sainte-Rose C. Shunt obstruction: a preventable complication? Pediatric Neurosurgery 1993; 19(3):156-64.

Schatlo 2013 \{published data only\}

Schatlo B, Hamed M, Grote A, Schramm J, Neuloh G. Gravity assisted vs. medium pressure valves for communicating 
hydrocephalus show similar valve-revision rates. Acta Neurochirurgica 2013; 155(10):1987-91.

\section{Sinha 2012 \{published data only\}}

Sinha A, Sharma A, Gupta C. Pediatric hydrocephalus: does the shunt device pressure selection affect the outcome? Journal of Indian Association of Pediatric Surgeons 2012; 17(2):54-7.

\section{Smely 1997 \{published data only\}}

Smely C, Van Velthoven V. Comparative study of two customary cerebrospinal fluid shunting systems in early childhood hydrocephalus. Acta Neurochirurgica 1997; 139(9):875-82.

Sotelo 2005 \{published data only\}

Sotelo J, Arriada N, Lopez MA. Ventriculoperitoneal shunt of continuous flow vs valvular shunt for treatment of hydrocephalus in adults. Surgical Neurology 2005; 63(3):197-203.

\section{Symss 2015 \{published data only\}}

Symss NP, Oi S. Is there an ideal shunt? A panoramic view of 110 years in CSF diversions and shunt systems used for the treatment of hydrocephalus: from historical events to current trends. Child's Nervous System 2015; 31(2):191-202.

\section{Villeda 1997 \{published data only\}}

Villeda CJM, Gómez AA, Mateos JH. Eficacia de la válvula tipo diafragma en hidrocefalia aguda. Archivos de Neurociencias 1997; 2(4):237-41.

\section{Wong 2012 \{published data only\}}

Wong JM, Ziewacz JE, Ho AL, Panchmatia JR, Bader AM, Garton $\mathrm{HJ}$, et al. Patterns in neurosurgical adverse events: cerebrospinal fluid shunt surgery. Neurosurgical Focus 2012; 33(5):E13.

\section{Xenos 2003 \{published data only\}}

Xenos C, Sgouros S, Natarajan K, Walsh AR, Hockley A. Influence of shunt type on ventricular volume changes in children with hydrocephalus. Journal of Neurosurgery 2003; 98(2):277-83.

\section{Xu 2013 \{published data only\}}

Xu H, Wang ZX, Chen DH, Zhang SL, Guo JF, Tan GW, et al. Effectiveness of programmable valves for hydrocephalus: a systematic review (provisional abstract). Chinese Journal of Evidence-Based Medicine 2013; 13(1):78-85.

\section{Xu 2013a \{published data only\}}

Xu H, Wang ZX, Liu F, Tan GW, Zhu HW, Chen DH. Programmable shunt valves for the treatment of hydrocephalus: a systematic review. European Journal of Paediatric Neurology 2013; 17(5):454-61.

\section{Additional references}

\section{Adams 1965}

Adams RD, Fischer CM, Hakim S, Ojemann RG, Sweet WH. Symptomatic occult hydrocephalus with "normal" cerebrospinal fluid pressure. A treatable syndrome. New England Journal of Medicine 1965; 273:117-26.

\section{Ames 1967}

Ames RH. Ventriculo peritoneal shunts in the management of hydrocephalus. Journal of Neurosurgery 1967; 27:525-9.

\section{Bradley 2015}

Bradley WG. CSF flow in the brain in the context of normal pressure hydrocephalus. American Journal of Neuroradiology 2015; 36:831-8.

\section{Campbell 2020}

Campbell M, McKenzie JE, Sowden A, Katikireddi SV, Brennan SE, Ellis S, et al. Synthesis without meta-analysis (SWiM) in systematic reviews: reporting guideline. BMJ 2020; 368:16890.

\section{Carey 1994}

Carey MC, Tullous MW, Walker ML. Chapter 12: Hydrocephalus: etiology, pathological effects, diagnosis, and natural history. In: Cheek WR, Marlin A, McLone DG, Reigel DH, Walker ML, editors(s). Pediatric Neurosurgery, Surgery of the Developing Nervous System. 3rd edition. Philadelphia (PA): W.B. Saunders Company, 1994:185-201.

\section{Champney 2016}

Champney T. Chapter 2: Blood vessels, meninges, and ventricles. In: Wiley Blackwell, editors(s). Essential Clinical Neuroanatomy. 1st edition. Chichester (UK): Wiley \& Sons, Ltd, 2016:20-38

\section{Cheng 2015}

Cheng HW, Hong WM, Mei ZJ, Wang XJ. Surgical management of non-communicating hydrocephalus in patients: meta-analysis and comparison of endoscopic third ventriculostomy and ventriculoperitoneal shunt. Journal of Craniofacial Surgery 2015; 26:481-6.

\section{Czosnyka 1998}

Czosnyka Z, Czosnyka M, Richard H, Pickard D. Posturerelated overdrainage: comparison of the performance of 10 hydrocephalus shunts in vitro. Neurosurgery 1998; 42(2):327-33.

\section{Drake 1995}

Drake J, Sainte RC. The shunt book. In: The Shunt Book. Cambridge (MA): Blackwell Science, 1995:3-12.

\section{Drake 2006}

Drake J, Chumas P, Kestle J, Pierre-Kahn A, Vinchon M, Brown J, et al. Late rapid deterioration after endoscopic third ventriculostomy: additional cases and review of literature. Journal of Neurosurgery 2006; 105(2):118-26.

\section{Esmonde 2002}

Esmonde T, Cooke S. Shunting for normal pressure hydrocephalus (NPH). Cochrane Database of Systematic Reviews 2002, Issue 3. [DOI: 10.1002/14651858.CD003157]

\section{ESPN 2018}

. 26th Congress of the European Society for Pediatric Neurosurgery (ESPN) Bonn-Germany, 6-9 May 2018. Childs Nervous System 2018; 34(5):995-1094. 


\section{GRADE Working Group 2004}

GRADE Working Group. Grading quality of evidence and strength of recommendations. BMJ 2004; 328(7454):1490.

\section{GRADEpro 2008 [Computer program]}

GRADE Working Group, McMaster University GRADEpro. Version 3.2 beta. Hamilton (ON): GRADE Working Group, McMaster University, 2008.

\section{Guyatt 2008}

Guyatt GH, Oxman AD, Vist GE, Kunz R, Falck-Ytter Y, Schünemann HJ, et al. GRADE: what is "quality of evidence" and why is it important to clinicians? BMJ (Clinical Research Ed) 2008; 336(7651):995-8. [DOI: 10.1136/bmj.39490.551019.BE]

\section{Guyatt 2011}

Guyatt G, Oxman AD, Akl EA, Kunz R, Vist G, Brozek J, et al. GRADE guidelines: 1 . Introduction - GRADE evidence profiles and summary of findings tables. Journal of Clinical Epidemiology 2011; 64(4):383-94. [DOI: 10.1016/ j.jclinepi.2010.04.026

\section{Hader 2002}

Hader W, Drake J, Cochrane D, Sparrow O, Johnson E, Kestle J. Death after late failure of third ventriculostomy in children. Report of three cases. Journal of Neurosurgery 2002; 97:211-5.

\section{Hagberg 1962}

Hagberg B. The sequelae of spontaneously arrested infantile hydrocephalus. Developmental Medicine and Child Neurology 1962; 4:583-7.

\section{Halperin 2015}

Halperin JJ, Kurlan R, Schwalb JM, Cusimano MD, Gronseth G, Gloss D. Practice guideline: Idiopathic normal pressure hydrocephalus: response to shunting and predictors of response. Neurology 2015; 85:2063-71.

\section{Han 2005}

Han CY, Backous DD. Basic principles of cerebrospinal fluid metabolism and intracranial pressure homeostasis. Otolaryngologic Clinics of North America 2005; 38(4):569-76.

\section{Hanlo 2003}

Hanlo P, Cinalli C, Vandertop WP, Faber JA, Bogescob L, Borgesen $\mathrm{S}$, et al. Treatment of hydrocephalus determined by the European Orbis Sigma Valve II survey: a multicenter prospective 5-year shunt survival study in children and adults in whom a flow-regulating shunt was used. Journal of Neurosurgery 2003; 99:52-7.

\section{Health Information Research Unit (HIRU)}

Health Information Research Unit (HIRU). Evidencebased health informatics. Search strategies for PsycINFO in OVID syntax. hiru.mcmaster.ca/hiru/ HIRU_Hedges_EMBASE_Strategies.aspx (accessed 14 December 2015).

\section{Higgins 2002}

Higgins JP, Thompson SG. Quantifying heterogeneity in a metaanalysis. Statistics in Medicine 2002; 21(11):1539-58. [DOI: 10.1002/sim.1186]

\section{Higgins 2003}

Higgins JP, Thompson SG, Deeks JJ, Altman DG. Measuring inconsistency in meta-analyses. BMJ (Clinical Research Ed) 2003; 327(7414):557-60. [DOI: 10.1136/bmj.327.7414.557]

\section{Higgins 2008}

Higgins JP, White IR, Wood AM. Imputation methods for missing outcome data in meta-analysis of clinical trials. Clinical Trials (London, England) 2008; 5(3):225-39.

\section{Higgins 2011a}

Higgins JP, Altman DG, Sterne JA, . Chapter 8: Assessing risk of bias in included studies. In: Higgins JP, Green S, editor(s). Cochrane Handbook for Systematic Reviews of Interventions. Version 5.1.0 (updated March 2011). The Cochrane Collaboration, 2011. Available from handbook.cochrane.org.

\section{Higgins 2011b}

Higgins JP, Deeks JJ, Altman DG, . Chapter 16: Special topics in statistics. In: Higgins JP, Green S, editor(s). Cochrane Handbook for Systematic Reviews of Interventions. Version 5.1.0 (updated March 2011). The Cochrane Collaboration, 2011. Available from handbook.cochrane.org.

\section{Higgins 2019}

Higgins JPT, Eldridge S, . Chapter 23: Including variants on randomized trials. In: Higgins JPT, Thomas J, Chandler J, Cumpston M, Li T, Page MJ, Welch VA (editors). Cochrane Handbook for Systematic Reviews of Interventions version 6.0 (updated July 2019). Cochrane 2019. Available from www.training.cochrane.org/handbook.

\section{Hirsch 1994}

Hirsch JP. Consensus: long term outcome in hydrocephalus. Child's Nervous System 1994; 10(1):64-9.

\section{ICD-9-CM 2004}

Centers for Disease Control and Prevention, National Center for Health Statistics. ICD-9-CM addenda, conversion table, and guidelines. www.cdc.gov/nchs/icd/ icd9cm_addenda_guidelines.htm\#conversion_table (accessed 6 May 2016).

\section{ISPN 2017}

. 45th Annual Meeting of International Society for Pediatric Neurosurgery, Denver, USA, 8-12 October, 2017. Childs Nervous System 2017; 33(10):1785-1853.

\section{ISPN 2018}

. 46th Annual Meeting of International Society for Pediatric Neurosurgery, Tel Aviv, ISRAEL, 7-11 October, 2018. Childs Nervous System 2018; 34(10):1997-2122.

\section{ISPN 2019}

.47th Annual Meeting of the International Society for Pediatric Neurosurgery. Childs Nervous System 2019; 35(10):1925-2020. 


\section{Jansen 1985}

Jansen J. A retrospective analysis 21 to 35 years after birth of hydrocephalic patients born from 1946 to 1955 . An overall description of the material and of the criteria used. Acta Neurologica Scandinavica 1985; 71:436-47.

\section{Jeng 2011}

Jeng S, Gupta N, Wrensch M, Zhao S, Wu YW. Prevalence of congenital hydrocephalus in California, 1991-2000. Pediatric Neurology 2011; 45(2):67-71.

\section{Kahle 2015}

Kahle KT, Kulkarni AV, , Warf BC. Hydrocephalus in children. Lancet 2016; 387(10020):788-99.

\section{Kaiser 1992}

Kaiser G, Bittel M. Preliminary experiences with the Orbis Sigma-System as a ventriculo peritoneal shunt. European Journal of Pediatric Surgery 1992; 2(3):186-7.

\section{Kausch 1908}

Kausch W. The treatment of hydrocephalus in infants [Die behandlung des hydrocephlus del cleinen kinder]. Langenbecks Archiv für Klinische Chirurgie 1908; 87:709-96.

\section{Kulkarni 2004}

Kulkarni AV, Rabin D, Drake JM. An Instrument to measure the health status of children with hydrocephalus: the Hydrocephalus Outcome Questionnaire. Journal of Neurosurgery 2004; 101(2 Suppl):134-40.

\section{Kulkarni 2007}

Kulkarni A, Shams I. Quality of life in children with hydrocephalus: results from the hospital for sick children, Toronto. Journal of Neurosurgery 2007; 107(5 Suppl):358-64.

\section{Kulkarni 2013}

Kulkarni A, Riva Cambrin J, Butler J, Browd SR, Drake JM, Holubkov R, et al. Outcomes of CSF shunting in children: comparison of Hydrocephalus Clinical Research Network cohort with historical controls. Journal of Neurosurgery (Pediatrics) 2013; 12(4):334-8.

\section{Kurtom 2007}

Kurtom KH, Magram G. Siphon regulatory devices: their role in the treatment of hydrocephalus. Neurosurgical Focus 2007; 22(4):E5.

\section{Landgraf 1999}

Langraft JM, Abetz L, Ware JE. Child's Health questionnaire. In: The CHQ User's Manual. Boston (MA): HealthAct, 1999.

\section{Laurence 1962}

Laurence KM, Coates S. The natural history of hydrocephalus. Detailed analysis of 182 unoperated cases. Archives of Disease in Childhood 1962; 37(194):345-62.

\section{Laurence 1967}

Laurence K, . Spontaneously arrested hydrocephalus results of the re-examination of 82 survivors from a series of 182 unoperated cases. Developmental Medicine and Child Neurology 1967; 9(13):4-13.

\section{Lee 2009}

Lee HS, Yoon SH. Hypothesis for lateral ventricular dilatation in communicating hydrocephalus: new understanding of the Monro-Kellie hypothesis in the aspect of cardiac energy transfer through arterial blood flow. Medical Hypotheses 2009; 2(7):174-7.

\section{Lefebvre 2011}

Lefebvre C, Manheimer E, Glanville J. Chapter 6: Searching for studies. In: Higgins JP, Green S, editor(s). Cochrane Handbook for Systematic Reviews of Interventions Version 5.1.0 (updated March 2011). The Cochrane Collaboration, 2011. Available from handbook.cochrane.org.

\section{Liberati 2009}

Liberati A, Altman DG, Tetzlaff J, Mulrow C, Gøtzsche PC, Ioannidis JP, et al. The PRISMA statement for reporting systematic reviews and meta-analyses of studies that evaluate health care interventions: explanation and elaboration. Journal of Clinical Epidemiology 2009; 62(10):e1-34. [DOI: 10.1371/ journal.pmed.1000100]

\section{Lifshutz 2001}

Liftshutzd J, Johnson W. History of hydrocephalus and its treatments. Neurosurgical Focus 2001; 11(2):1-5.

\section{Limbrick 2014}

, Baird LC, , Riva-Cambrin J, Flannery AM, Pediatric Hydrocephalus Systematic Review and Evidence-Based Guidelines Task Force. Pediatric hydrocephalus: systematic literature review and evidence-based guidelines. Part 4: Cerebrospinal fluid shunt or endoscopic third ventriculostomy for the treatment of hydrocephalus in children. Journal of Neurosurgery (Pediatrics) 2014; 14 Suppl 1:30-4.

\section{Lumenta 1990}

Lumenta CB, Roosen N, Dietrich U. Clinical experience with a pressure-adjustable valve SOPHY in the management of hydrocephalus. Child's Nervous System 1990; 6(5):270-4.

\section{Mancall 2011}

Mancall EL, Brock DG. Section I.5: Ventricular system and cerebrospinal fluid. In: Standring S, Crossman A, editors(s). Gray's Clinical Neroanatomy. The Anatomic Basis for Clinical Neuroscience. 1st edition. Philadelphia, (PA): Elsevier Saunders, 2011:98-108.

\section{Matson 1949}

Matson D. A new operation for the treatment of communicating hydrocephalus: report of a case secondary to generalized meningitis. Journal of Neurosurgery 1949; 6(3):238-47.

\section{Mccullough 1990}

McCullough D. History of the treatment of hydrocephalus. In: Scott MR, editors(s). Hydrocephalus. Vol. 3. Baltimore (MD): Williams \& Wilkins, 1990:1-10. 


\section{McKenzie 2019}

McKenzie JE, Brennan SE. Chapter 12: Synthesizing and presenting findings using other methods. In: Higgins JPT, Thomas J, Chandler J, Cumpston M, Li T, Page MJ, Welch VA (editors). Cochrane Handbook for Systematic Reviews of Interventions version 6.0 (updated July 2019). Cochrane, 2019. Available from www.training.cochrane.org/handbook.

\section{Miyake 2016}

Miyake H. Shunt devices for the treatment of adult hydrocephalus: recent progress and characteristics. Neurologia Medico-Chirurgica 2016; 56:274-83.

\section{Munch 2012}

Munch TN, Rostgaard K, Hee Rasmussen ML, Wohlfahrt J, Juhler M, Melbye M. Familial aggregation of congenital hydrocephalus in a nationwide cohort. Brain 2012; 135:2409-15.

\section{O'Neill 1961}

O'Neill EM. Normal head growth and the prediction of head size in infantile hydrocephalus. Archives of Disease in Childhood 1961; 36(187):241-52.

\section{Ojemann 1968}

Ojemann RG. Initial experience with the Hakin valve for ventriculovenous shunt. Technical note. Journal of Neurosurgery 1968; 28:283-7.

\section{Patwardhan 2005}

Patwardhan R, Nanda A. Implanted ventricular shunts in the United States: the billion dollar a year cost of hydrocephalus treatment. Neurosurgery 2005; 56:139-44.

\section{Peters 2014}

Peters JN, Mahajan JK, Bawa M, Sahu PK, Rao KL. Factors affecting quality of life in early childhood in patients with congenital hydrocephalus. Child's Nervous System 2014; 30(5):867-71.

\section{Petre 2010}

Petre C, Portillo S, Fernandez A. Hydrocephalus in children [Hidrocefalia en niños]. In: Basso A, Carrizo G, Mezadri JJ, Goland J, Socolovsky M, editors(s). Neurocirugia: Aspectos Clínicos y Quirúrgicos. 1st edition. Buenos Aires (Argentina): Corpus, Rosario, 2010:111-9.

\section{Pikus 1997}

Pikus HJ, Levy ML, Gans W, Mendel E, McComb JG. Outcome, cost analysis, and long term follow up in pre-term infants with massive grade IV germinal matrix hemorrhage and progressive hydrocephalus. Neurosurgery 1997; 40(5):983-9.

\section{Post 1985}

Post EM. Currently available shunt systems: a review. Neurosurgery 1985; 16:257-60.

\section{Pudenz 1957}

Pudenz R, Russell F, Hurd A, Shelden C. Ventriculo auriculostomy; a technique for shunting cerebrospinal fluid into the right auricle. Preliminary report. Journal of Neurosurgery $1957 ; \mathbf{1 4}(2): 171-9$

\section{Review Manager 2014 [Computer program]}

Nordic Cochrane Centre, The Cochrane Collaboration Review Manager 5 (RevMan 5). Version 5.3. Copenhagen: Nordic Cochrane Centre, The Cochrane Collaboration, 2014.

\section{Rohde 2009}

Rohde V, Haberl EJ, Ludwig H, Thomale U. First experiences with an adjustable gravitational valve in childhood hydrocephalus. Journal of Neurosurgery (Pediatrics) 2009; 3(2):90-3.

\section{Schroeder 1999}

Schroeder HW, Gaab M. Intracranial endoscopy. Neurosurgical Focus 1999; 6(4):e1.

\section{Schünemann 2011}

Schünemann HJ, Oxman AD, Higgins JP, Vist GE, Glasziou P, Guyatt GH. Chapter 11: Presenting results and 'Summary of findings' tables. In: Higgins JP, Green S, editor(s), Cochrane Handbook for Systematic Reviews of Interventions. Version 5.1.0 (updated March 2011). The Cochrane Collaboration, 2011. Available from handbook.cochrane.org.

\section{Sciubba 2007}

Sciubba DM, Lin LM, Woodworth GF, McGirt MJ, Carson B, Jallo G. Factors contributing to the medical costs of cerebrospinal fluid shunt infection treatment in pediatric patients with standard shunt components compared with those in patients with antibiotic impregnated components. Neurosurgical Focus 2007; 22(4):e9.

\section{Shannon 2011}

Shannon CN, Simon TD, Reed GT, Franklin FA, Kirby RS, Kilgore ML, et al. The economic impact of ventriculoperitoneal shunt failure. Journal of Neurosurgery (Pediatrics) 2011; 8(6):593-9.

\section{Smith 2004}

Smith E, Butler W, Barkell F. In-hospital mortality rates after ventriculoperitoneal shunt procedures in United States, 1998 to 2000: relation to hospital and surgeon volume of care. Journal of Neurosurgery (Pediatrics) 2004; 100(2):90-7.

\section{Stein 2008}

Stein SC, Guo W. Have we made progress in preventing shunt failure? A critical analysis. Journal of Neurosurgery (Pediatrics) 2008; 1(1):40-7.

\section{Texakalidis 2019}

Texakalidis P, Tora M, Wetzel J, Chern J. Endoscopic third ventriculostomy versus shunt for pediatric hydrocephalus: a systematic literature review and meta-analysis. Childs Nervous System 2019; 35(8):1283-93.

\section{Tudor 2015}

Tudor KI, Tudor M, McCleery J, Car J. Endoscopic third ventriculostomy (ETV) for idiopathic normal pressure hydrocephalus (iNPH). Cochrane Database of Systematic Reviews 2015, Issue 7. [DOI: 10.1002/14651858.CD010033.pub2] [CD010033] 


\section{Tully 2014}

Tully H, Dobyns W. Infantile hydrocephalus: a review of epidemiology, classification and causes. European Journal of Medical Genetics 2014; 57(8):359-68.

\section{Ware 2012}

.SF-36 ${ }^{\circledR}$ Health Survey update. Spine 2000; 15(24):3130-9.

\section{Watson 1994}

Watson AD. The Delta valve: a physiologic shunt system. Child's Nervous System 1994; 10:224-30.

\section{Whytt 1768}

Whytt R. Hippocrates de morbis. In: Observations on the Dropsy in the Brain. Edinburgh (UK): Balfour, 1768:4.

\section{Williams 1990}

Williams A. EuroQol: a new facility for the measurement of health-related quality of life. Health Policy 1990; 16:199-208.

\section{CHARACTERISTICS OF STUDIES}

Characteristics of included studies [ordered by study ID]

\section{Wolburg 2010}

Wolburg H, Paulus W. Choroid plexus: biology and pathology. Acta Neuropathologica 2010; 119(1):75-88.

\section{Yashon 1963}

Yashon D. Prognosis in infantile hydrocephalus; past and present. Journal of Neurosurgery 1963; 20:105-11.

\section{Ziebell 2013}

Ziebell M, Wetterslev J, Tisell M, Gluud C, Juhler M. Flowregulated versus differential pressure-regulated shunt valves for adult patients with normal pressure hydrocephalus. Cochrane Database of Systematic Reviews 2013, Issue 5. [DOI: 10.1002/14651858.CD009706.pub2]

* Indicates the major publication for the study

Drake 1998

\section{Study characteristics}

\begin{tabular}{ll} 
Methods & Study design: parallel-group randomised trial \\
Study dates: October 11993 to October 311995 \\
Setting: inpatient, multicentre, international \\
Country: Canada, United States, France \\
\hline
\end{tabular}

Participants Inclusion criteria:

Patients between birth and 18 years of age, newly diagnosed hydrocephalus with documented ventriculomegaly in CT, MRI or ultrasonographic image scans and requiring a first ventriculo-peritoneal CSF shunt insertion.

Exclusion criteria:

1- previous indwelling CSF shunt (patients with subcutaneous reservoirs for aspiration or ventricular catheters draining externally or to the subcutaneous scalp were eligible);

2- active abdominal or CSF infection;

3- diffuse spread of tumour in the subarachnoid space;

4- marked prematurity with skin at risk for erosion from shunt hardware;

5- systemic disorders precluding shunt insertion;

6- septated loculations within the ventricular system requiring more than one shunt;.

7- Dandy Walker malformation;

8- arachnoid cyst as a cause of hydrocephalus;

9- inability to be monitored for 1 year baseline.

Sample size: 344 participants randomised

Group 1: Delta valve 115

Group 2: Standard valve 114 
Drake 1998 (Continued)

Group 3: Orbis-Sigma valve 115

Age (years):

Group 1: Delta valve: mean 1.83 years, median 0.2 years

Group 2: Standard valve: mean 1.65 years; median 0.21 years

Group 3: Orbis-Sigma valve: mean 1.56 years; median 0.4 years

$\operatorname{Sex}(M / F):$

Group 1: Delta valve 64/51

Group 2: Standard valve 70/44

Group 3: Orbis-Sigma valve 57/58

Hydrocephalus cause

Group 1: Delta valve: intraventricular haemorrhage $19.1 \%$, myelomeningocele $24.3 \%$, tumour $8.7 \%$, aqueduct stenosis $7.8 \%$, CSF infection $6.1 \%$, head injury $2.6 \%$, two or more causes $9.6 \%$, other $9.6 \%$, unknown $12.2 \%$

Group 2: Standard valve: intraventricular haemorrhage $25.9 \%$, myelomeningocele $24.1 \%$, tumour $8.0 \%$, aqueduct stenosis $8.0 \%$, CSF infection $4.5 \%$, head injury $1.8 \%$, two or more causes $6.3 \%$, other $11.6 \%$, unknown $9.8 \%$

Group 3 Orbis-Sigma valve: intraventricular haemorrhage $28.1 \%$, myelomeningocele $15.8 \%$, tumour $10.5 \%$, aqueduct stenosis $5.3 \%$, CSF infection $5.3 \%$, head injury $0.0 \%$, two or more causes $10.5 \%$, other $13.2 \%$, unknown $11.4 \%$

Previous surgery:

Group 1 Delta valve: subcutaneous reservoir $6.1 \%$, ventricular drain $8.0 \%$

Group 2 Standard valve: subcutaneous reservoir $6.1 \%$, ventricular drain $11.9 \%$

Group 3 Orbis-Sigma valve: subcutaneous reservoir $7.1 \%$, ventricular drain $18.0 \%$

Group $1(n=115)$ : Delta valve (Medtronic PS Medical): a standard diaphragm valve with an additional anti-syphon device that consists of paired flexible diaphragms, which narrow an orifice as the shunt pressure becomes negative

Group 2 ( $n=114)$ : Standard (conventional) valve

Group $3(n=115)$ : Cordis Orbis-Sigma valve. This is a flow-regulation valve with a flexible diaphragm which moves along a piston of variable diameter. It operates through three stages, so that the pressure curve is sigmoid in shape which limits flow by progressively narrowing the flow orifice with increasing pressure, as a pressure-sensitive ring moves along a variable-diameter rod with a single opening pressure of approximately $5 \mathrm{~cm} \mathrm{H}_{2} \mathrm{O}$.

Co-interventions: The details of the surgical technics and postoperative care were established according to the discretion of the surgeon and were recorded (including the prophylactic use of antibiotics, hair removal, the site of hardware insertion, the use of technical aids such as ventriculoscopes, hardware configurations, opening pressure designations for the standard and Delta valves, and postoperative head elevation or compressive dressings). Suggestions from the manufacturers for insertion techniques were solicited at the beginning of the trial and were distributed to all participating centres.

\section{Treatment failure}

How measured: defined as shunt malfunction due to shunt obstruction, shunt over-drainage, loculated ventricles, or shunt infection. Each of these end points were defined by specific criteria in the trial protocol (see below). An adjudication committee, blinded to the treatment group, reviewed each patient's imaging studies, operative notes and hospital discharge summary when a primary end point was reported. 
Time points reported: complete follow-up

Treatment failure [shunt obstruction]

Definition:

1. If a patient has at least one symptom or sign and at least one positive ancillary test

Symptoms: headache, nausea, vomiting, decreased level of consciousness, irritability, decreased school performance, loss of developmental milestones

Signs: papilledema, bulging fontanelle, nuchal rigidity, 6th nerve(s) paresis, loss of upward gaze, new seizures (or increased seizure frequency), increasing head circumference, fluid tracking along the shunt tubing, umbilication of the shunt reservoir, inability to depress the shunt reservoir. Fluid accumulation around the burr hole site in the early weeks following surgery will not be considered indicative of shunt failure unless it is extreme and progressive or results in leakage of CSF through the wound. Small fluid collections are common and normally resolve spontaneously.

Ancillary tests:

(a) CT scan, ultrasound, or MRI scan showing enlarged ventricles compared to the 3-month study or ventricles that have failed to decrease in size compared to the preoperative study (normalisation of ventricle size is not a mandatory criterion for shunt function)

(b) Disruption or migration of the shunt system on plain radiographs

(c) Radionucleotide or iodinated contrast study showing shunt obstruction

(d) ICP monitoring showing persistent elevation of pressure with or without plateau waves

(e) Shunt tap in which fluid cannot be aspirated or high pressure is recorded or symptoms/signs of shunt obstruction are relieved

2. When there are no symptoms or signs of shunt obstruction but the ventricles are increased in size, shunt obstruction is said to have occurred if there is no clinical or radiographic suggestion that atrophy is the cause of the ventricular enlargement.

3. A CSF leak that does not resolve and which requires a shunt revision

4. In the rare event of an emergent shunt revision without any ancillary tests, or revision prior to the 3month follow-up scan, obstruction will be judged to be present or absent using clinical information and the operative findings (by the Monitoring/Adjudication Committee)

Time points measured: clinical evaluation at 3 months, 1 year, and yearly until the end of the study

Time points reported: complete follow-up

\section{Treatment failure [shunt over-drainage]}

Definition: in the presence of (a) subdural fluid collections - large subdural fluid collections associated with brain compression or symptoms and signs otherwise indicative of shunt obstruction; or (b) slit ventricle syndrome - smaller than normal ventricles associated with postural headache, chronic headache, intermittent headache of an incapacitating nature and documentation of one of the following: (1) transient enlargement of the ventricles as seen on imaging; (2) extreme negative pressure with associated headache in the upright position; (3) sustained elevations of pressure above normal associated with headache

Time points measured: clinical evaluation at 3 months, 1 year, and yearly until the end of the study

Time points reported: complete follow-up

\section{Treatment failure [loculated ventricles]}

Definition: The presence of a loculated portion of a ventricular system that is enlarged above normal and compressing surrounding brain and that requires reoperation

Time points measured: clinical evaluation at 3 months, 1 year, and yearly until the end of the study 
Time points reported: complete follow-up

\section{Treatment failure [shunt infection]}

Definition:

1. In the presence of purulent discharge through the wound or erosion of the shunt material through the skin

2. In the presence of one of the following symptoms or signs with at least one of the following ancillary tests

Symptoms and signs: fever, meningismus, wound erythema, abdominal pain and/or distention, abdominal mass or peritonitis

Ancillary tests: culture or identification of organisms on Gram stain of CSF taken from shunt lumen or abdominal fluid collection, if present, withdrawn under sterile conditions or from purulent material around the shunt

Time points measured: clinical evaluation at 3 months, 1 year, and yearly until the end of the study

Time points reported: complete follow-up

\section{Adverse events [surgical complications]}

How measured: not specified. The following events were included in this outcome (obtained from the Hospital Discharge Form): transient CSF leak, intracranial haemorrhage, new or increased neurological deficit, neck injury, chest injury, abdominal injury, inadvertent skin perforation along shunt tract, wound dehiscence, postoperative subcutaneous fluid collection, perioperative death, and "other" complications.

Time points measured: time of hospital discharge

Time points reported: time of hospital discharge

\section{Mortality for all causes}

How measured: not specified. Cause of death was recorded on a death form and classified as probably related to shunt malfunction; neurologic, but probably not related to the shunt malfunction; or nonneurological.

Time points measured: complete follow-up

Time points reported: complete follow-up

Quality of life: not reported

Ventricular size reduction: not reported

Head circumference: not reported dation and The Science Council of British Columbia (Burnaby Canada), Mr. and Mrs. PA Woodward's Foundation (Vancouver Canada) British Columbia's Children's Hospital Telethon Clinical Investigative Initiatives (Vancouver, Canada) British Columbia's Medical Services Foundation (Vancouver, Canada) Spina Bifida Association of Canada (Winnipeg, Canada) and Spina Bifida Association of British Columbia (Surrey, Canada)

\section{Declarations of interest}

\section{Notes}

$$
\text { Not reported }
$$


Drake 1998 (Continued)

no uniform standards for these designations, typically their pressure differentials are 5, 10 and $15 \mathrm{~mm}$ $\mathrm{H}_{2} \mathrm{O}$, respectively. Despite the variations in the standard valve design, the pressure/flow characteristics are very similar.

2) Assessment at 3 months, 1 year and yearly until the end of the study were performed by the attending neurosurgeon during an outpatient hospital visit by the patient, using a specific form. Follow-up included imaging studies at 3 months and thereafter according to the surgeons' individual practices. A 12-month scan was encouraged if possible. A completed telephone follow-up form was required at months 18 and 30. A minimum follow-up of 1 year was required.

The details of the surgical techniques and postoperative care were established according to the discretion of the surgeon, including the prophylactic use of antibiotics, hair removal, the site of hardware insertion, the use of technical aids such as ventriculoscopes, hardware configurations, opening pressure designations for the standard and Delta valves, and postoperative head elevation or compressive dressings. Suggestions from the manufacturers for insertion techniques were solicited the beginning of the trial and were distributed to all participating centres.

\section{Risk of bias}

\begin{tabular}{|c|c|c|}
\hline Bias & Authors' judgement & Support for judgement \\
\hline $\begin{array}{l}\text { Random sequence genera- } \\
\text { tion (selection bias) }\end{array}$ & Low risk & $\begin{array}{l}\text { "Patients were stratified by centre and by age (less than or more than } 6 \mathrm{mo} \text { ), } \\
\text { and the randomisation scheme was blocked with randomly varying block } \\
\text { size." }\end{array}$ \\
\hline $\begin{array}{l}\text { Allocation concealment } \\
\text { (selection bias) }\end{array}$ & Low risk & "Consecutive opaque envelopes located in each operating room." \\
\hline $\begin{array}{l}\text { Blinding of participants } \\
\text { and personnel (perfor- } \\
\text { mance bias) } \\
\text { Subjective outcomes: } \\
\text { treatment failure, adverse } \\
\text { events, quality of life }\end{array}$ & High risk & $\begin{array}{l}\text { Study personnel were aware of the type of valve that the patient received. The } \\
\text { shunts were distinctly different in shape, can be easily palpated through the } \\
\text { skin, and were radio-opaque. }\end{array}$ \\
\hline $\begin{array}{l}\text { Blinding of participants } \\
\text { and personnel (perfor- } \\
\text { mance bias) } \\
\text { Objective outcomes: mor- } \\
\text { tality, head circumference, } \\
\text { ventricular size }\end{array}$ & Low risk & $\begin{array}{l}\text { No information about blinding available. Outcomes were likely to be unaffect- } \\
\text { ed by blinding. }\end{array}$ \\
\hline
\end{tabular}

\begin{tabular}{|c|c|c|}
\hline $\begin{array}{l}\text { Blinding of outcome as- } \\
\text { sessment (detection bias) } \\
\text { Subjective outcomes: } \\
\text { treatment failure, adverse } \\
\text { events, quality of life }\end{array}$ & Low risk & $\begin{array}{l}\text { "Determination of outcome will be made by the neurosurgeon in charge of the } \\
\text { care of the child at the time of the malfunction. The outcome will be reviewed } \\
\text { by the adjudication committee, which will be blinded to the type of shunt in } \\
\text { place." }\end{array}$ \\
\hline $\begin{array}{l}\text { Blinding of outcome as- } \\
\text { sessment (detection bias) } \\
\text { Objective outcomes: mor- } \\
\text { tality, head circumference, } \\
\text { ventricular size }\end{array}$ & Low risk & $\begin{array}{l}\text { No information about blinding available. Outcomes were likely to be unaffect- } \\
\text { ed by blinding. }\end{array}$ \\
\hline
\end{tabular}

Incomplete outcome data Low risk

(attrition bias)

Eight patients did not complete the minimum 1-year follow-up period. " $8 / 344$

All outcomes patients did not complete the minimum 1-year follow-up period." 
Drake 1998 (Continued)

\begin{tabular}{|c|c|c|}
\hline $\begin{array}{l}\text { Selective reporting (re- } \\
\text { porting bias) }\end{array}$ & High risk & $\begin{array}{l}\text { Some outcomes listed in the protocol were not reported, e.g. change in ven- } \\
\text { tricular size, hospital stay. Data on mortality was not disaggregated by group. }\end{array}$ \\
\hline
\end{tabular}

Other bias Low risk No other sources of bias were detected.

Garcia 1988

\section{Study characteristics}

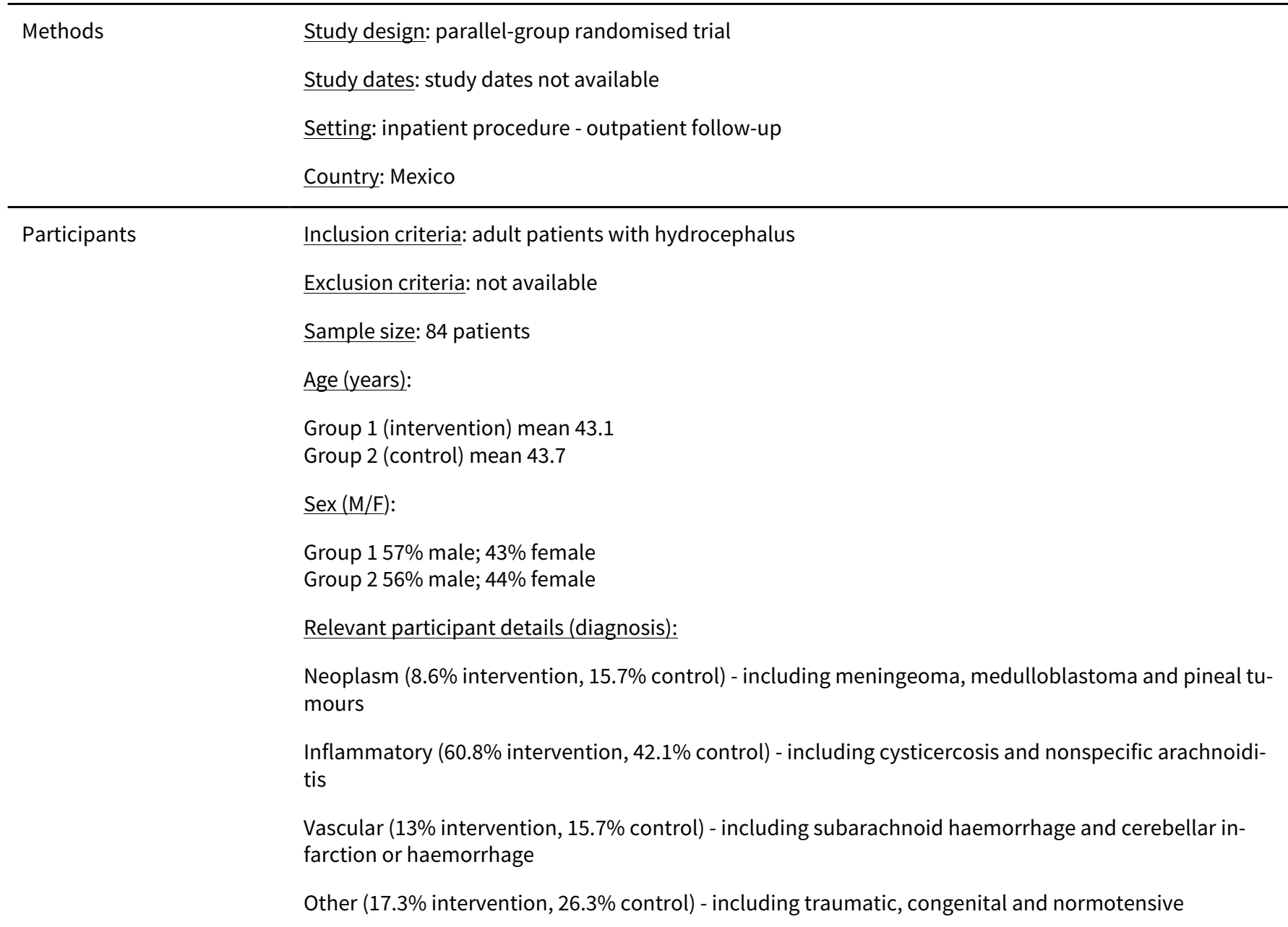

Interventions $\quad$ Group $1(n=46)$ : Biomed distal valve (antireflux). This group received 54 valves. Seven patients received more than one valve (15 systems due to dilatation or failure).

Group 2 ( $n=38)$ : Cordis-Hakim proximal valve (ball in cone). This group received 42 valves. Eight patients received more than one valve (23 systems due to dilatation or failure).

Co-interventions: all patients received antibiotic prophylaxis (doxycycline and chloramphenicol) and a computerised tomography before the surgery, at the 7th and 15th and 30th day postoperatively.

Outcomes

\section{Treatment failure}

How measured: surgical re intervention for changing the shunting device due to obstruction, infection, or over-drainage G1: 9 valves in 8 patients (14\%) G2: 17 valves in 11 patients (46\%)

Time points measured: 
Garcia 1988 (Continued)

G1: $12.7 \pm 4.6$ months

G2: $9.4 \pm 7.3$ months.

Time points reported:

G1: 1 to 21 months.

G2: 1 to 36 months

Subgroups: none

\section{Adverse events}

How measured: CSF culture (infection) or computerised tomography scan - > also reported under treatment failure.

Time points measured: "early" or "late" (mean follow-up 4 months, range 1-24 months)

Time points reported: idem

Subgroups: none

\section{Mortality}

How measured: not specified

Time points measured: complete follow-up (mean follow-up 4 months, range 1-24 months)

Time points reported: complete follow-up (mean follow-up 4 months, range 1-24 months)

Subgroups: none

Quality of life: not reported

Ventricular size reduction: not reported

Head circumference: not reported

\begin{tabular}{ll}
\hline Funding sources & Not available \\
\hline Declarations of interest & Not available \\
\hline Notes & $\begin{array}{l}\text { This study reported a subjective clinical scale: "excellent" (normal function), "good" (few symptoms, } \\
\text { independent in daily life activities), "regular" (greater symptoms, minor impairment in daily activities), } \\
\text { "bad" (major impairment and dependent on continuous care including hospital care). }\end{array}$
\end{tabular}

\section{Risk of bias}

\begin{tabular}{lll}
\hline Bias & Authors' judgement & Support for judgement \\
\hline $\begin{array}{l}\text { Random sequence genera- } \\
\text { tion (selection bias) }\end{array}$ & Unclear risk & Quote: "a prospective randomised study" \\
& No other information available \\
\hline $\begin{array}{l}\text { Allocation concealment } \\
\text { (selection bias) }\end{array}$ & Unclear risk & Quote: "a prospective randomised study" \\
\hline $\begin{array}{l}\text { Blinding of participants } \\
\text { and personnel (perfor- } \\
\text { mance bias) }\end{array}$ & High risk & No other information available \\
$\begin{array}{l}\text { Subjective outcomes: } \\
\text { treatment failure, adverse } \\
\text { events, quality of life }\end{array}$ & & No information about blinding available, however personnel was aware of the \\
\end{tabular}


Garcia 1988 (Continued)

Blinding of participants and personnel (performance bias)

Objective outcomes: mor-

tality, head circumference, ventricular size
Low risk No information about blinding available, however personnel was aware of the type of shunt being placed during the procedure. Outcomes were likely to be unaffected by blinding.

\section{Blinding of outcome as- sessment (detection bias) Subjective outcomes: treatment failure, adverse events, quality of life}

\section{High risk}

No information about blinding available, however personnel was aware of the type of shunt being placed during the procedure
Blinding of outcome as- Low risk sessment (detection bias)

Objective outcomes: mortality, head circumference, ventricular size
No information about blinding available, however personnel was aware of the type of shunt being placed during the procedure. Outcomes were likely to be unaffected by blinding.

\begin{tabular}{lll}
\hline $\begin{array}{l}\text { Incomplete outcome data } \\
\text { (attrition bias) } \\
\text { All outcomes }\end{array}$ & Low risk & All outcomes: follow-up data was complete for all participants. \\
\hline $\begin{array}{l}\text { Selective reporting (re- } \\
\text { porting bias) }\end{array}$ & Unclear risk & $\begin{array}{l}\text { Insufficient information to permit judgement of 'Low risk' or 'High risk' (no } \\
\text { protocol available) }\end{array}$ \\
\hline Other bias & Low risk & No other sources of bias were identified. \\
\hline
\end{tabular}

Khan 2010

\section{Study characteristics}

\begin{tabular}{l} 
Methods \\
Study design: parallel-group randomised trial \\
Study dates: study dates not available \\
Setting: inpatient procedure - outpatient follow-up \\
Country: India \\
\hline Inclusion criteria: participants between 20 days to 8 months, with progressive hydrocephalus with or \\
without neural tube defect (NTD). The most common cause of hydrocephalus in children without NTD \\
was aqueductal stenosis. \\
Exclusion criteria: patients having meningitis, ventriculitis, hydrocephalus secondary to mass lesions, \\
intraventricular haemorrhage, peritoneal infection or peritoneal adhesions \\
Sample size: 40 eligible were randomly allocated using random number table. Patients: 26 NTD hydro- \\
cephalus and 14 without NTD \\
Age (years): \\
Group A: Anti-siphon group (20 days to 8 months, mean 3.5$)$ \\
Group B: Non-antisyphon (25 days to 8 months) \\
Sex (M/F) Group A: 6 female, 14 male; Group B: 5 female, 15 male \\
Relevant participant details:
\end{tabular}


Khan 2010 (Continued)

Group A:

Neural tube defect 35\%

Low shunt pressure $85 \%$

Medium shunt pressure $15 \%$

Group B:

Neural tube defect 35\%

Slow shunt pressure $80 \%$

Medium shunt pressure 20\%

Interventions

Group A $(n=20)$ : with anti-syphon device (Vygon)

Group B ( $n=20)$ : without anti-syphon device (Chhabra and Ceredrain)

Outcomes

Treatment failure

How measured: number of participants with shunt block, shunt infection and over-drainage

Time points measured: 3 and 6 months

Time points reported: unclear (possibly 6 months)

\section{Adverse events (morbidity)}

How measured: number of participants with shunt block, shunt infection and over-drainage

Time points measured: 3 and 6 months

Time points reported: unclear (possibly 6 months)

\section{Head circumference}

How measured: Occipito frontal circumference (OFC)

Time points measured: at discharge, 3 and 6 months postoperative

Time points reported: at discharge, 3 and 6 months postoperative

\section{Ventricular size}

How measured: Modified Evan's index (MEI) assessed by computerised tomography

Time points measured: at discharge, 3 and 6 months postoperative

Time points reported: at discharge, 3 and 6 months postoperative

Mortality for all causes: not reported

Quality of life: not reported

\begin{tabular}{ll}
\hline Funding sources & Not available \\
\hline Declarations of interest & Not available \\
\hline Notes & $\begin{array}{l}\text { No description was made about why surgeons chose different pressures and little information is pro- } \\
\text { vided on the types of valves used. } \\
\text { Contact information: drrizwanahmadkhan@yahoo.co.in (R.A. Khan). }\end{array}$ \\
\hline Risk of bias & Authors' judgement Support for judgement \\
\hline Bias &
\end{tabular}


Khan 2010 (Continued)

$\begin{array}{ll}\text { Random sequence genera- } \quad \text { Low risk } & \text { "Patients were randomly allocated to the two groups of the shunts using ran- } \\ \text { tion (selection bias) } & \text { dom number table." }\end{array}$

$\begin{array}{ll}\begin{array}{l}\text { Allocation concealment } \\ \text { (selection bias) }\end{array} & \text { Unclear risk }\end{array} \quad \begin{aligned} & \text { No information available. Insufficient information to permit judgement of 'Low } \\ & \text { risk' or 'High risk' }\end{aligned}$

\begin{tabular}{ll}
\hline $\begin{array}{l}\text { Blinding of participants } \\
\text { and personnel (perfor- }\end{array}$ & High risk \\
$\begin{array}{l}\text { mance bias) } \\
\text { Subjective outcomes: } \\
\text { treatment failure, adverse } \\
\text { events, quality of life }\end{array}$ & $\begin{array}{l}\text { No information about blinding available, however blinding was unlikely due to } \\
\text { the visibly different interventions. }\end{array}$ \\
\hline $\begin{array}{l}\text { Blinding of participants } \\
\text { and personnel (perfor- } \\
\text { mance bias) }\end{array}$ & Low risk \\
Objective outcomes: mor- \\
tality, head circumference, \\
ventricular size
\end{tabular}

\begin{tabular}{|c|c|c|}
\hline $\begin{array}{l}\text { Blinding of outcome as- } \\
\text { sessment (detection bias) }\end{array}$ & High risk & $\begin{array}{l}\text { No information about blinding available, however blinding was unlikely due to } \\
\text { the visibly different interventions. }\end{array}$ \\
\hline
\end{tabular}

Subjective outcomes: the visibly different interventions.

treatment failure, adverse

events, quality of life

$\begin{array}{ll}\text { Blinding of outcome as- } & \text { Low risk } \\ \text { sessment (detection bias) } & \text { No information about blinding available. Outcomes were likely to be unaffect- } \\ \text { ed by blinding. }\end{array}$

Objective outcomes: mor-

tality, head circumference, ventricular size

\begin{tabular}{lll}
\hline $\begin{array}{l}\text { Incomplete outcome data } \\
\text { (attrition bias) } \\
\text { All outcomes }\end{array}$ & Low risk outcomes: outcome data were available for all participants. \\
\hline $\begin{array}{l}\text { Selective reporting (re- } \\
\text { porting bias) }\end{array}$ & Unclear risk & $\begin{array}{l}\text { Insufficient information to permit judgement of 'Low risk' or 'High risk' (no } \\
\text { protocol available) }\end{array}$ \\
\hline Other bias & Low risk & No other sources of bias were identified. \\
\hline
\end{tabular}

\section{Liniger 2003}

\section{Study characteristics}

\begin{tabular}{ll}
\hline Methods & Study design: parallel-group quasi-randomised trial \\
& Study dates: start date: 1992 - end date: 1996 \\
& Setting: inpatient procedure - outpatient follow-up \\
& Country: Switzerland \\
\hline Participants & Inclusion criteria: 27 patients between 36 days to 6 months, with progressive hydrocephalus with docu- \\
mented ventriculomegaly by computed CT scan, MRI or ultrasonographic image scans and requiring a \\
first ventriculoperitoneal CSF shunt insertion
\end{tabular}


Liniger 2003 (Continued)

\section{Exclusion criteria: not available}

Sample size: 27 randomised participants

Age:

Group A: median age at surgery in months 3.4 (1 - 5 months)

Group B: median age at surgery in months 2.2 ( $0-4$ months)

Sex $(M / F)$ : not available

Relevant participant details:

Group A:

- Congenital/acquired hydrocephalus $8 / 3$

- Mean head circumference: $43 \mathrm{~cm}$

- Percentiles $10>97.1=97$

- Ultrasound mean thalamus-occipital horn distance: $42 \mathrm{~mm}$

- Mean with of lateral ventricles: 43

- Mean (range) of IVP: 185 (100-240) $\mathrm{mm} \mathrm{H}_{2} \mathrm{O}$

- CT/MRI mean age Evan's index 0.46 (0.3-0.6)

- Mean frontal/occipital brain mantle: $15.5 / 7 \mathrm{~mm}$

Group B:

- Congenital/acquired hydrocephalus $11 / 3$

- Mean head circumference: $41 \mathrm{~cm}$

- Percentiles $10>97.1=50$

- Ultrasound mean thalamus-occipital horn distance: 27

- Mean with of lateral ventricles: 47

- Mean (range) of IVP: $172(100-260) \mathrm{mm} \mathrm{H}_{2} \mathrm{O}$

- CT/MRI mean age Evan's index 0.32 (0.2-0.5)

- Mean frontal/occipital brain mantle: $17 / 11 \mathrm{~mm}$

Previous Surgery: no

Details of valve selection: not available
Group A ( $n=11)$ : “conventional medium pressure valve" (PS Medical flow control valve, Goleta CA, medium pressure)

Group B ( $n=16)$ : "Anti-siphon valve" (PS Medical Delta, level 1.0, medium-low pressure)

Common indications: Ventriculoperitoneal shunt was implanted on the right or the side of the larger ventricle.

\section{Treatment failure}

How measured: defined as the number of patients with revisions and the type of revisions. Shunt revisions were recorded and compared in respect of the valve inserted and in respect of the development of slit ventricles.

Time points measured: 3 and 6 months

Time points reported: unclear (possibly 6 months)

\section{Adverse events}

How measured: Slit Ventricle (SV) were defined according to the radiological impression of slit-like or collapsed lateral ventricles in CT or MRI scans. Slit Ventricle syndrome was defined as SV with recurrent 
clinical signs of intracranial hypertension, namely severe headaches and emesis, and slow or no refill of the valve.

Time points measured: at 6 months after shunt insertion (early), and at 6 years of age (late)

Time points reported: at 6 months after shunt insertion (early), and at 6 years of age (late)

\section{Ventricular size reduction:}

How measured: width of lateral ventricles (CT scan or MRI)

Time points measured: 3 and 6 months

Time points reported: unclear (possibly 6 months)

\section{Head circumference:}

How measured: head circumference in $\mathrm{cm}$ at a corrected age of 7 years

Time points measured: 3 and 6 months

Time points reported: unclear (possibly 6 months)

Mortality for all causes: not reported

Quality of life: not reported

\begin{tabular}{ll}
\hline Funding sources & Not available \\
\hline Declarations of interest & Not available \\
\hline Notes & $\begin{array}{l}\text { Other outcomes included IVP, psychomotor development (unclear scale of measurement), ultrasound } \\
\text { imaging, mean Evan's index, mean frontal/occipital brain mantle in mm. }\end{array}$ \\
\hline
\end{tabular}

\section{Risk of bias}

\begin{tabular}{lll}
\hline Bias & Authors' judgement & Support for judgement \\
\hline $\begin{array}{l}\text { Random sequence genera- } \\
\text { tion (selection bias) }\end{array}$ & High risk & $\begin{array}{l}\text { Quote: "infants with progressive hydrocephalus were allocated alternately to } \\
\text { either Group A (conventional medium pressure valve) or Group B (Delta valve } \\
\text { level 1)". It was not clear that a random sequence was used (quasi-randomisa- } \\
\text { tion). }\end{array}$ \\
\hline $\begin{array}{l}\text { Allocation concealment } \\
\text { (selection bias) }\end{array}$ & Unclear risk & $\begin{array}{l}\text { No information available. See above. Insufficient information to permit judge- } \\
\text { ment of 'Low risk' or 'High risk' }\end{array}$
\end{tabular}

\begin{tabular}{|c|c|c|}
\hline $\begin{array}{l}\text { Blinding of participants } \\
\text { and personnel (perfor- }\end{array}$ & High risk & $\begin{array}{l}\text { No information about blinding available, however blinding was unlikely due to } \\
\text { the visibly different interventions. }\end{array}$ \\
\hline
\end{tabular}
mance bias)

Subjective outcomes:

treatment failure, adverse

events, quality of life

\begin{tabular}{|c|c|c|}
\hline $\begin{array}{l}\text { Blinding of participants } \\
\text { and personnel (perfor- } \\
\text { mance bias) } \\
\text { Objective outcomes: mor- } \\
\text { tality, head circumference, } \\
\text { ventricular size }\end{array}$ & Low risk & $\begin{array}{l}\text { No information about blinding available. Outcomes were likely to be unaffect- } \\
\text { ed by blinding. }\end{array}$ \\
\hline $\begin{array}{l}\text { Blinding of outcome as- } \\
\text { sessment (detection bias) }\end{array}$ & High risk & $\begin{array}{l}\text { No information about blinding available, however blinding was unlikely due to } \\
\text { the visibly different interventions. }\end{array}$ \\
\hline
\end{tabular}


Liniger 2003 (Continued) Subjective outcomes: treatment failure, adverse events, quality of life

$\begin{array}{ll}\begin{array}{l}\text { Blinding of outcome as- } \\ \text { sessment (detection bias) } \\ \text { Objective outcomes: mor- } \\ \text { tality, head circumference, }\end{array} & \begin{array}{l}\text { No information about blinding available. Outcomes were likely to be unaffect- } \\ \text { ed by blinding. }\end{array} \\ \text { ventricular size } & \end{array}$

\begin{tabular}{|c|c|c|}
\hline $\begin{array}{l}\text { Incomplete outcome data } \\
\text { (attrition bias) }\end{array}$ & Unclear risk & $\begin{array}{l}\text { No information available. Insufficient information to permit judgement of 'Low } \\
\text { risk' or 'High risk' }\end{array}$ \\
\hline
\end{tabular}

\begin{tabular}{|c|c|c|}
\hline $\begin{array}{l}\text { Selective reporting (re- } \\
\text { porting bias) }\end{array}$ & High risk & $\begin{array}{l}\text { Insufficient information to permit judgement of 'Low risk' or 'High risk' (no } \\
\text { protocol available). However, outcome data were presented at selective time } \\
\text { points and frequently not disaggregated by group (e.g. shunt failure defined as } \\
\text { elective versus emergency revisions). }\end{array}$ \\
\hline
\end{tabular}

Other bias Low risk No other sources of bias were detected.

\section{Pollack 1999}

\section{Study characteristics}

Methods
Study design: parallel-group randomised trial
Study dates: randomisation June 29, 1993 to December 14, 1995
Setting: inpatient procedure, outpatient follow-up, multicentre, national
Country: United States

\section{Participants}

Inclusion criteria: patients who required placement of ventriculoperitoneal or ventriculo-atrial shunt for the treatment of hydrocephalus (first placement or replacement)

Exclusion criteria: patients under treatment with drugs for anticoagulation, coagulopathies, active infection, valve explantation because of infection within the past 3 months, requiring two valves at the same time, any contraindication for ventriculo-atrial or ventriculo-peritoneal shunting, expected lifespan less than 24 months, and unwillingness or inability to return for required follow-up evaluation at the investigational site

\section{Sample size: 377 participants randomised}

Group 1: Experimental: 194

Group 2: Control: 183

Age (years):

Group 1: Experimental: median $14(+/-23)$

Group 2: Control: median $12(+/-23)$

\section{$\underline{\operatorname{Sex}(M / F)}$ :}

Group 1: $59 \%$ male, $41 \%$ female

Group 2: $54 \%$ male, $46 \%$ female 
Pollack 1999 (Continued)
Diagnosis:
Group 1: communicating hydrocephalus 59\%, non-communicating $41 \%$
Group 2: communicating hydrocephalus 57\%, non-communicating $43 \%$

Interventions

Group 1: Experimental $(n=194)$ : Codman-Hakim programmable valve system (Codman/Johnson \& Johnson, Raynham, MA)

Group 2: Control ( $n=183$ ): Any non-programmable valve pressure with fixed pressure available on the market: Delta (Medtronic PS Medical, Goleta Ca): $10 \%$, other Medtronic PS Medical: $57 \%$, other Codman/Johnson \& Johnson: 8\%, Orbis-Sigma (Cordis, Miami Lakes, FL): 3\%, others: $22 \%$

Co-interventions: The study required that the programmable valve in all patients in whom it had been placed be reprogrammed after any imaging study because of concern that the valve setting would alter in the magnetic field. Other than this instruction, decisions regarding the programmable valve pressures were left entirely to the respective investigator's judgement.

\section{Primary outcomes}

\section{Treatment failure [shunt system failure]:}

Definition: a surgical intervention to the original shunt system on-study, for any reason, was considered a shunt system failure.

Primary outcome measure: survival of the shunt system.

Time points measured: clinical evaluations 1, 3, 6, 12, 18 and 24 months

Time points reported: 24 months

Treatment failure [infection necessitating explantation]:

Definition: not reported

Time points measured: clinical evaluations 1, 3, 6, 12, 18 and 24 months

Time points reported: Not reported (probably 104 weeks)

\section{Adverse events:}

Definition: "complications included the following: haemorrhage, malabsorption of CSF, subdural haematoma/hygroma, neurological deficit, catheter perforation, foreign body reaction, infection, and "other" complications".

Time points measured: clinical evaluations 1, 3, 6, 12, 18 and 24 months and radiological images at 3 , 12 and 24 months

Time points reported: Not reported (probably 104 weeks)

\section{Adverse events [subdural hematoma/hygroma]:}

Definition: not reported

Time points measured: clinical evaluations $1,3,6,12,18$ and 24 months

Time points reported: Not reported (probably 104 weeks)

Mortality for all causes: not reported

Quality of life: not reported

\section{Ventricular size reduction}

Definition: ventricle size was reported as big, average, small, or slit, according to qualitative assessment of image studies (computed tomography, MRI, or ultrasound studies). 
Pollack 1999 (Continued)

Time points measured: evaluated at 3, 12 and 24 months with radiological images (ultrasound, CT scan and MRI)

Time points reported: radiological images at 3,12 and 24 months

Head circumference: not reported

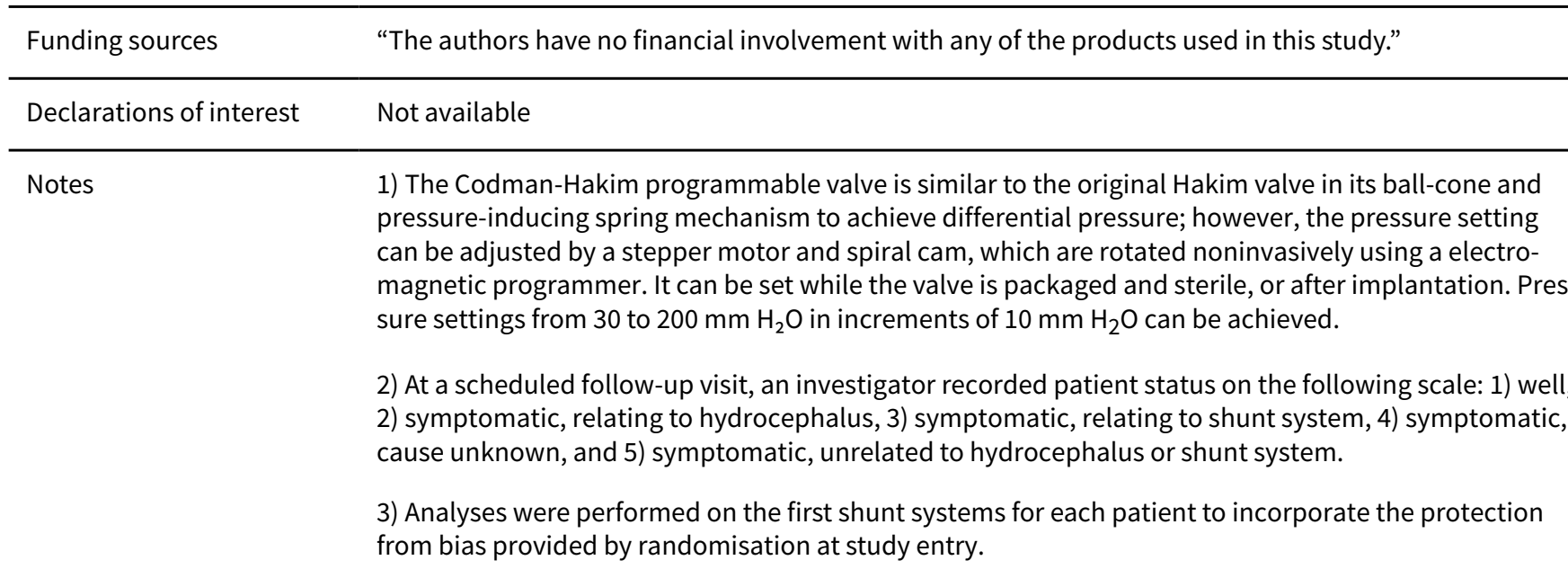

\section{Risk of bias}

\section{Bias \\ Authors' judgement Support for judgement}

Random sequence genera- Unclear risk tion (selection bias)

"Randomisation was stratified by study site, and within each site randomisation was stratified further by patient shunt history. Patients with a history of one or more previous shunts before placement of a study valve were classified as "replacement" patients. Each of these two groups was randomised separately within each site to maintain balance design".

No information available

\begin{tabular}{lll}
\hline $\begin{array}{l}\text { Allocation concealment } \\
\text { (selection bias) }\end{array}$ & Unclear risk & See above. No other information available \\
\hline $\begin{array}{l}\text { Blinding of participants } \\
\text { and personnel (perfor- } \\
\text { mance bias) }\end{array}$ & High risk & $\begin{array}{l}\text { Participants and personnel were not blinded. There was no detail on co-inter- } \\
\text { ventions. }\end{array}$ \\
$\begin{array}{l}\text { Subjective outcomes: } \\
\text { treatment failure, adverse } \\
\text { events, quality of life }\end{array}$ & \\
\hline
\end{tabular}

Blinding of participants Low risk

Participants and personnel were not blinded. There was no detail on co-interand personnel (perforventions. Outcomes were unlikely to be affected by blinding.

mance bias)

Objective outcomes: mor-

tality, head circumference, ventricular size

\begin{tabular}{|c|c|c|}
\hline $\begin{array}{l}\text { Blinding of outcome as- } \\
\text { sessment (detection bias) }\end{array}$ & High risk & Participants and personnel were not blinded. \\
\hline $\begin{array}{l}\text { Subjective outcomes: } \\
\text { treatment failure, adverse } \\
\text { events, quality of life }\end{array}$ & & \\
\hline
\end{tabular}


Pollack 1999 (Continued) sessment (detection bias)

Objective outcomes: mor-

tality, head circumference, ventricular size

Incomplete outcome data Low risk

(attrition bias)

Low risk

Participants and personnel were not blinded. Outcomes were unlikely to be affected by blinding.

7 patients (2/194 experimental, 5/183 control) were lost to follow-up, 19 died

(11/194 experimental, 8/183 control) before the 24 -month follow-up point was

All outcomes reached. More than $90 \%$ of patients completed the 3,12 and 24-month postoperative evaluations.

\begin{tabular}{|c|c|c|}
\hline $\begin{array}{l}\text { Selective reporting (re- } \\
\text { porting bias) }\end{array}$ & Unclear risk & $\begin{array}{l}\text { Insufficient information to permit judgement of 'Low risk' or 'High risk' (no } \\
\text { protocol available) }\end{array}$ \\
\hline
\end{tabular}

Other bias Low risk No other sources of bias were identified.

\section{Warf 2005}

\section{Study characteristics}

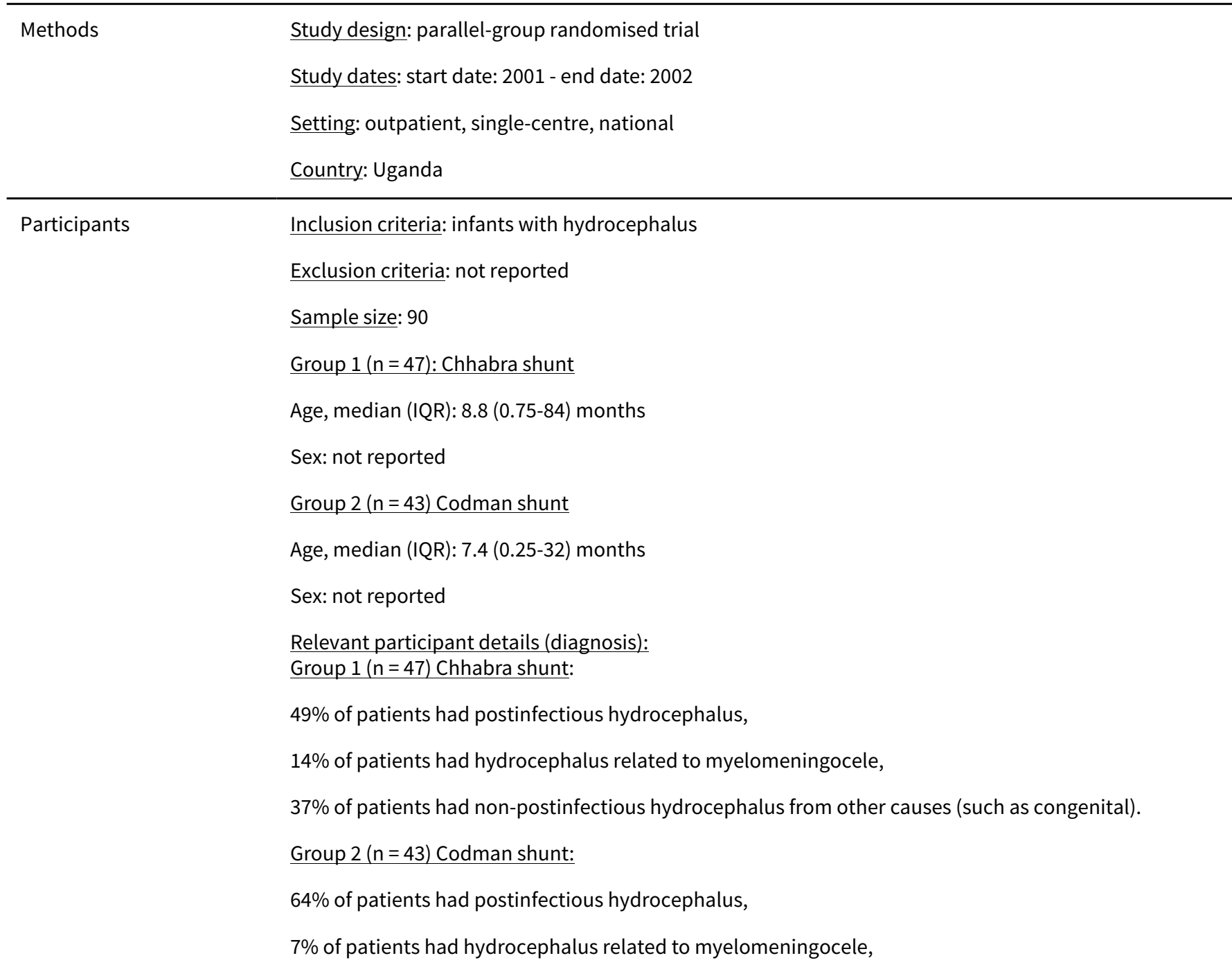


Warf 2005 (Continued)

$29 \%$ of patients had non-postinfectious hydrocephalus from other causes (such as congenital).

Interventions

Group 1 ( $n=47)$ : Patients received a Chhabra shunt. The Chhabra shunt (G. Surgiwear Ltd., India) is an unified shunt system that incorporates a proximal slit-in-spring valve. The Chhabra system is available in three pressure type models. A medium pressure system allows a flow of $20 \mathrm{~mL} / \mathrm{hr}$ at a pressure gradient $10 \mathrm{~cm}$ of water.

Group 2 ( $n=43$ ): Patients received a Codman VP shunt. Codman-Hakim Micro Precision Valve (Medos S. A. [Johnson \& Johnson Co.], Switzerland) is a unified shunt using the Hakim Precision Valve. Codman-Hakim Micro Precision Valves are fixed pressure valves and are available in 5 different opening pressure ranges. The operating pressures of the valve unit have been determined with a flow rate of $10-$ $25 \mathrm{~mL} \mathrm{H} \mathrm{H}_{2} \mathrm{O}$ per hour. The valve is classified by its operating pressure with a specified flow rate and not by its opening and closing pressures.

Co-interventions: "Patients were positioned for right frontal or right occipital shunt placement. An occipital approach was used with placement of the catheter in the occipital horn, with the exception of four Chhabra and one Codman shunts, which were placed frontally in the frontal horn. The shunt valve was flushed and tested to confirm an appropriate closing pressure. The shunt was tunnelled subcutaneously between the incisions. A pinpoint of dura was cauterised and punctured after removing bone, when necessary, and the ventricular catheter was passed. Flow was confirmed, a cerebrospinal fluid sample was obtained, and the catheter was secured to the valve connector (for the Chhabra) or the integral reservoir connector (for the Codman) with a 2-0 silk tie. The valve construct was then secured to the dura or pericranial tissue with a 3-0 silk stitch."

\section{Treatment failure}

How measured: valve malfunction and infection

Time points measured: 1 week and 3, 6, and 12 months postoperatively

Time points reported: 12 months

Subgroups: none

\section{Adverse events}

How measured: infections, obstruction and migration

Time points measured: 1 week and at 3, 6, and 12 months postoperatively

Time points reported: 12 months

Subgroups: none

\section{Mortality for all causes}

How measured: not reported

Time points measured: 1 week and at 3, 6, and 12 months postoperatively

Time points reported: 1 and 12 months

Subgroups: none

Quality of life: not reported

Ventricular size reduction: not reported

Head circumference: not reported (only as a baseline characteristic)

Funding sources International Federation for Spina Bifida and Hydrocephalus

CURE International (which funds CURE Children's Hospital of Uganda) 
Warf 2005 (Continued)

Declarations of interest The author has no financial interest that relates in any way to this report.

Notes

The report of the study included a "Group 2" which consisted of 105 consecutive patients in whom only Chhabra shunts were placed over the course of 9 months. This was prior to March 2002, in which no Codman shunts were available during this period.

\section{Risk of bias}

\section{Bias Authors' judgement Support for judgement}

Random sequence genera- High risk tion (selection bias)

\begin{abstract}
"Therefore, the type of VP shunt selected for use was random, based on either coin toss at the time of operation or availability and was in no way influenced by clinical criteria."

While there was a component of random sequence, the distribution of participants was also dependent on valve availability.
\end{abstract}

Allocation concealment Unclear risk $\quad$ No information available about allocation concealment
(selection bias)

\begin{tabular}{|c|c|c|}
\hline $\begin{array}{l}\text { Blinding of participants } \\
\text { and personnel (perfor- }\end{array}$ & High risk & $\begin{array}{l}\text { No information about blinding available, however personnel was aware of the } \\
\text { type of shunt being placed during the procedure. }\end{array}$ \\
\hline
\end{tabular}

mance bias)

Subjective outcomes:

treatment failure, adverse

events, quality of life

No information about blinding available, however personnel was aware of the

Blinding of participants Low risk
and personnel (perfor-
mance bias)
Objective outcomes: mor-
tality, head circumference,
ventricular size

Blinding of outcome assessment (detection bias) Subjective outcomes: treatment failure, adverse events, quality of life
No information about blinding available, however personnel was aware of the type of shunt being placed during the procedure. Outcomes were likely to be unaffected by blinding.

\section{Blinding of outcome as- Low risk} sessment (detection bias) Objective outcomes: mortality, head circumference, ventricular size

\section{High risk} No information about blinding available, however
type of shunt being placed during the procedure.

Incomplete outcome data High risk
(attrition bias)

All outcomes

\begin{abstract}
No information about blinding available, however personnel was aware of the type of shunt being placed during the procedure. Outcomes were likely to be unaffected by blinding.
\end{abstract}

\begin{abstract}
"Patients with no known problem but who were lost to follow up prior to 1 year postoperatively were eliminated from analysis." 4/47 participants were lost in the Chhabra group and $7 / 43$ participants were lost in the Codman group. Unbalanced attrition (9\% vs 16\%)
\end{abstract}

No protocol available. Head circumference and ventricular size were assessed at follow-up but they were not reported as outcomes (no information as to whether they were prespecified).

Selective reporting (re- Unclear risk porting bias)

No other sources of bias were detected.

\section{CSF: cerebrospinal fluid}


CT: computed tomography

ICP: intracranial pressure

IQR: interquartile range

IVP: intraventricular pressure

MEl: modified Evan's index

M/F: male/female

MRI: magnetic resonance imaging

NTD: neural tube defect

OFC: occipito frontal circumference

SV: slit ventricle

vs: versus

Characteristics of excluded studies [ordered by study ID]

\begin{tabular}{|c|c|}
\hline Study & Reason for exclusion \\
\hline Agarwal 2018 & Retrospective study that reviewed medical records \\
\hline ASERNIP-S 2014 & Health technology assessment of Miethke proSA $A^{\circledR}$ adjustable gravitational shunt \\
\hline Baird 2014 & Systematic review \\
\hline Beez 2014 & Retrospective study that reviewed medical records \\
\hline Czosnyka 1990 & Physiological study on valve mechanics \\
\hline Czosnyka 2000 & Letter \\
\hline Czosnyka 2002 & Physiological study on valve mechanics \\
\hline Decq 1995 & Retrospective study that reviewed medical records \\
\hline Del Bigio 1998 & Systematic review of in vitro studies \\
\hline Drake 1996 & Duplicate \\
\hline Drake 1998a & Narrative review \\
\hline Drake 2000 & Duplicate \\
\hline Eymann 2007 & Prospective study with no control group \\
\hline Felix 1983 & Prospective study with no control group \\
\hline Gruber 1984 & Prospective study with no control group \\
\hline Haberl 2009 & Prospective study with no control group \\
\hline Higashi 1994 & Case series comparing different valves \\
\hline Hoshide 2017 & Editorial \\
\hline Ito 2012 & Systematic review \\
\hline Jain 2005 & Case series \\
\hline Kiefer 2000 & Case series comparing different valves \\
\hline
\end{tabular}




\begin{tabular}{|c|c|}
\hline Study & Reason for exclusion \\
\hline Legat 1996 & Non-randomised comparative study \\
\hline Li 2017 & Systematic review \\
\hline Lund-Johansen 1994 & Case series comparing different valves \\
\hline Mbabazi-Kabachelor 2019 & Randomised study that assessed the effects of antibiotic-impregnated shunts \\
\hline Meling 2005 & Prospective study with no control group \\
\hline Portnoy 1976 & In vitro study \\
\hline Rasul 2012 & Systematic review \\
\hline Sainte-Rose 1993 & Case series \\
\hline Schatlo 2013 & Retrospective study \\
\hline Sinha 2012 & Randomised trial of a shunt at different pressures \\
\hline Smely 1997 & Case series \\
\hline Sotelo 2005 & $\begin{array}{l}\text { Wrong intervention (shunt of continuous flow did not include a valve device). Study population in- } \\
\text { cluded normotensive hydrocephalus. }\end{array}$ \\
\hline Symss 2015 & Retrospective study \\
\hline Villeda 1997 & Case series \\
\hline Wong 2012 & Systematic review \\
\hline Xenos 2003 & Observational study \\
\hline Xu 2013 & Systematic review \\
\hline Xu 2013a & Systematic review \\
\hline
\end{tabular}

DATA AND ANALYSES

Comparison 1. Standard valve vs anti-syphon valve

\begin{tabular}{lllll}
\hline Outcome or subgroup title & $\begin{array}{l}\text { No. of } \\
\text { studies }\end{array}$ & $\begin{array}{l}\text { No. of par- } \\
\text { ticipants }\end{array}$ & Statistical method & Effect size \\
\hline 1.1 Mortality & 1 & Risk Difference (M-H, Random, 95\% Cl) & Totals not selected \\
\hline
\end{tabular}


Analysis 1.1. Comparison 1: Standard valve vs anti-syphon valve, Outcome 1: Mortality

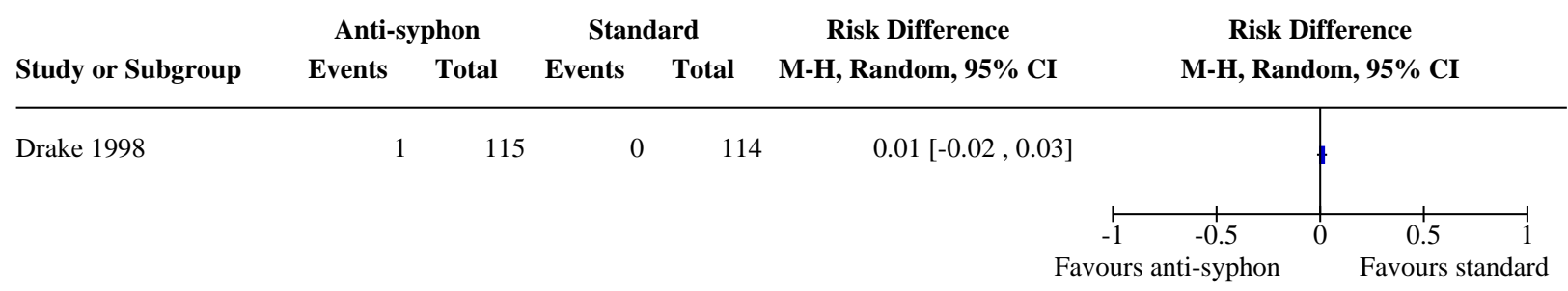

Comparison 2. Different types of standard valves

\begin{tabular}{lllll}
\hline Outcome or subgroup title & $\begin{array}{l}\text { No. of } \\
\text { studies }\end{array}$ & $\begin{array}{l}\text { No. of } \\
\text { partici- } \\
\text { pants }\end{array}$ & Statistical method & Effect size \\
\hline 2.1 Treatment failure & 2 & Risk Ratio $(\mathrm{M}-\mathrm{H}$, Random, $95 \% \mathrm{Cl})$ & Totals not selected \\
\hline 2.2 Mortality & 2 & Risk Difference $(\mathrm{M}-\mathrm{H}$, Random, $95 \% \mathrm{Cl})$ & Totals not selected \\
\hline
\end{tabular}

Analysis 2.1. Comparison 2: Different types of standard valves, Outcome 1: Treatment failure

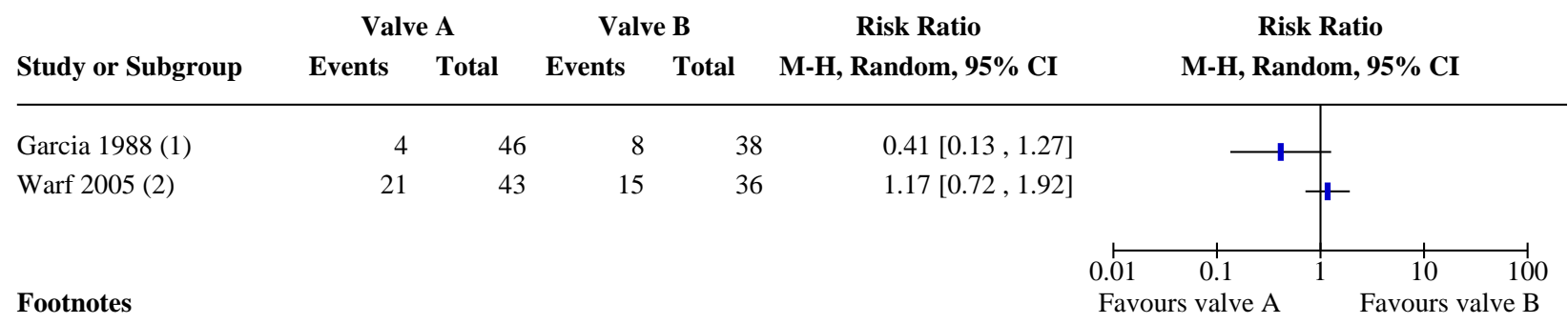

(1) Valve A: standard distal cleft. Valve B: Hakim ball-in-cone.

(2) Valve A: Chabbra. Valve B: Codman-Hakim

Analysis 2.2. Comparison 2: Different types of standard valves, Outcome 2: Mortality

\begin{tabular}{|c|c|c|c|c|c|c|}
\hline & & & $\mathbf{V a}$ & & Risk Difference & Risk Difference \\
\hline Study or Subgroup & Events & Total & Events & Total & M-H, Random, 95\% CI & M-H, Random, 95\% CI \\
\hline
\end{tabular}

\begin{tabular}{lllllr}
\hline Garcia 1988 (1) & 4 & 46 & 0 & 38 & $0.09[-0.00,0.18]$ \\
Warf 2005 (2) & 6 & 43 & 6 & 36 & $-0.03[-0.19,0.13]$ \\
Warf 2005 (3) & 1 & 43 & 0 & 36 & $0.02[-0.04,0.09]$
\end{tabular}

Footnotes

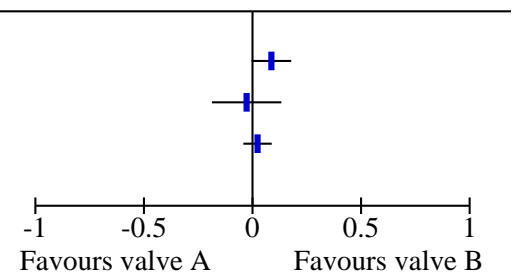

(1) Valve A: standard distal cleft. Valve B: Hakim ball-in-cone.

(2) Late deaths: Valve A: Chabbra. Valve B: Codman-Hakim.

(3) Early deaths: Valve A: Chabbra. Valve B: Codman-Hakim. 
Comparison 3. Standard valve versus constant flow valve

\begin{tabular}{lllll}
\hline Outcome or subgroup title & $\begin{array}{l}\text { No. of } \\
\text { studies }\end{array}$ & $\begin{array}{l}\text { No. of partici- } \\
\text { pants }\end{array}$ & Statistical method & Effect size \\
\hline 3.1 Treatment failure & 1 & 229 & Risk Ratio (M-H, Random, 95\% Cl) & $1.08[0.79,1.47]$ \\
\hline
\end{tabular}

Analysis 3.1. Comparison 3: Standard valve versus constant flow valve, Outcome 1: Treatment failure

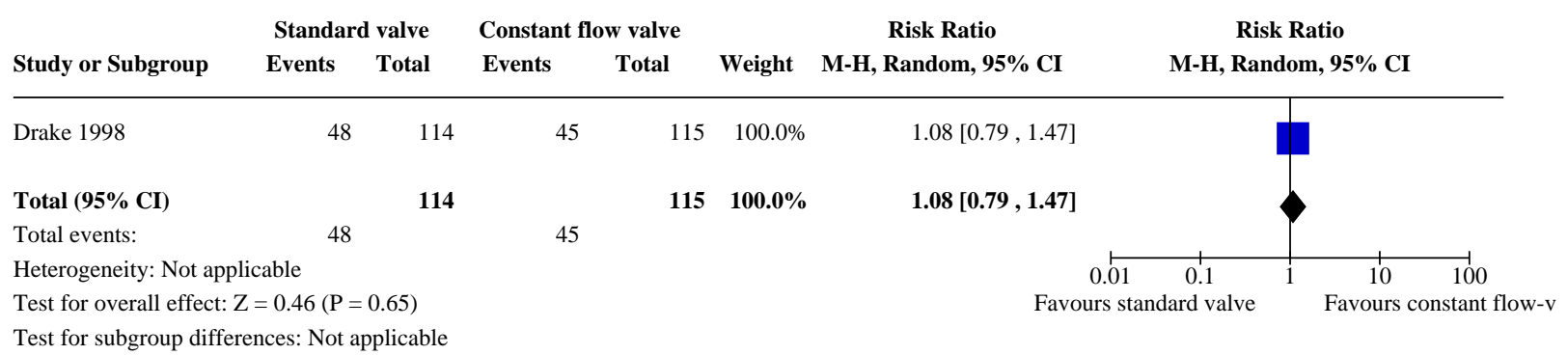

Comparison 4. Programmable valve versus non-programmable valve

\begin{tabular}{lllll}
\hline Outcome or subgroup title & $\begin{array}{l}\text { No. of } \\
\text { studies }\end{array}$ & $\begin{array}{l}\text { No. of par- } \\
\text { ticipants }\end{array}$ & Statistical method & Effect size \\
\hline 4.1 Treatment failure & 1 & 377 & Risk Ratio (M-H, Random, 95\% Cl) & $1.02[0.84,1.24]$ \\
\hline 4.2 Adverse events & 1 & 377 & Risk Ratio (M-H, Random, 95\% Cl) & $0.97[0.44,2.15]$ \\
\hline
\end{tabular}

Analysis 4.1. Comparison 4: Programmable valve versus non-programmable valve, Outcome 1: Treatment failure

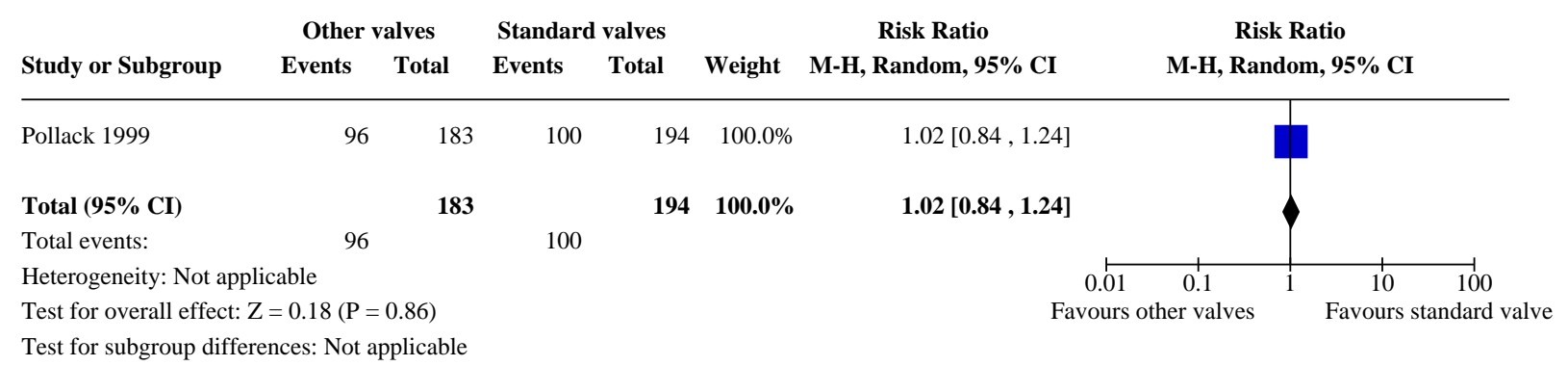


Analysis 4.2. Comparison 4: Programmable valve versus non-programmable valve, Outcome 2: Adverse events

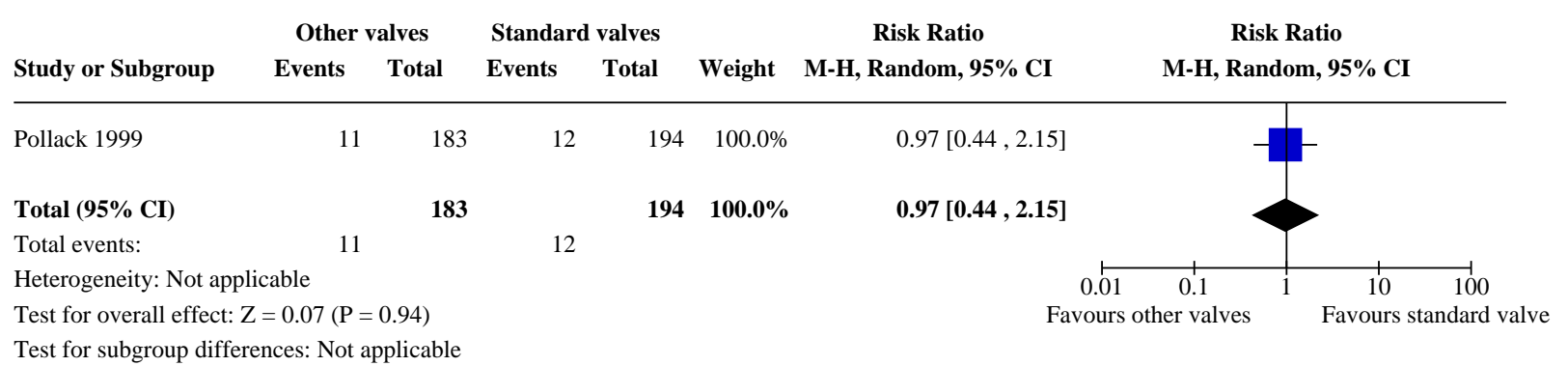

\section{APPENDICES}

\section{Appendix 1. Search strategies}

\begin{tabular}{|c|c|}
\hline Database and date & Search algorithm \\
\hline MEDLINE & 1 exp Hydrocephalus/ \\
\hline Ovid MEDLINE(R) & 2 hydrocephal*.ti,ab. \\
\hline In-Process \& Other Non- & 31 or 2 \\
\hline Indexed & 4 (convention* adj3 (valve* or shunt $\left.{ }^{\star}\right)$ ).ti,ab. \\
\hline Citations, Ovid & 5 (traditional $^{\star}$ adj3 $\left(\right.$ valve $^{\star}$ or shunt $\left.\left.{ }^{\star}\right)\right)$. ti,ab. \\
\hline MEDLINE(R) & 6 (conservativ* adj3 (valve* or shunt $\left.{ }^{\star}\right)$ ).ti,ab. \\
\hline Daily and Ovid & 7 (programm* adj3 (valve* or shunt $\left.{ }^{\star}\right)$ ).ti,ab. \\
\hline MEDLINE(R) & $8\left(\right.$ standard $^{\star}$ adj3 $\left(\right.$ valve $^{\star}$ or shunt $\left.\left.^{\star}\right)\right) . t i$, ab. \\
\hline$<1946$ to & 9 (cerebrospinal $^{\star}$ adj3 (valve or shunt $\left.\left.^{\star}\right)\right)$.ti,ab. \\
\hline \multirow[t]{12}{*}{ 12.02.2020> } & 10 (cerebro spinal $^{\star}$ adj3 $\left(\right.$ valve $^{\star}$ or shunt $\left.{ }^{\star}\right)$ ).ti,ab. \\
\hline & 11 (ventriculoperiton ${ }^{\star}$ adj3 (valve* or shunt $\left.^{\star}\right)$ ).ti,ab. \\
\hline & 12 (ventriculo periton* adj3 (valve* or shunt $\left.{ }^{\star}\right)$ ).ti,ab. \\
\hline & 13 (pressure adj3 (valve* or shunt $\left.{ }^{\star}\right)$ ).ti,ab. \\
\hline & 14 (shunt $^{\star}$ adj4 valve ${ }^{\star}$ ).ti,ab. \\
\hline & 15 (endosc ${ }^{\star}$ adj3 ventriculost $\left.{ }^{\star}\right) . t i, a b$. \\
\hline & 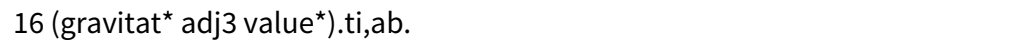 \\
\hline & 17 ventriculost $^{\star} . t i, a b$. \\
\hline & 184 or 5 or 6 or 7 or 8 or 9 or 10 or 11 or 12 or 13 or 14 or 15 or 16 or $17(11754)$ \\
\hline & 19 exp Cerebrospinal Fluid Shunts/ \\
\hline & 20 exp Surgical Instruments/ \\
\hline & 21 exp Equipment Design/ \\
\hline
\end{tabular}


2219 or 20 or 21

2318 or 22

243 and 23

25 systematic review.mp.

26 systematic review*.ti,ab.

27 meta analys ${ }^{\star} . t i, a b$.

28 meta?nalys ${ }^{\star}$.ti,ab.

2925 or 26 or 27 or 28

3024 and 29

31 Randomized Controlled Trial.pt.

32 Controlled Clinical Trial.pt.

33 randomi?ed.ab,ti.

34 placebo.ab,ti.

35 drug therapy.sh.

36 randomly.ab,ti.

37 trial.ab,ti.

38 groups.ab,ti.

3931 or 32 or 33 or 34 or 35 or 36 or 37 or 38

40 Animals/ not (Humans/ and Animals/)

4139 not 40

4224 and 41

\section{The Cochrane}

\#1 [mh hydrocephalus]

\section{Library}

14.02 .2020
\#2 hydrocephal ${ }^{*}: \mathrm{ti}, \mathrm{ab}$

\#3 \#1 or \#2

\#4 (valve* or shunt $\left.{ }^{\star}\right):$ ti,ab

\#5 (ventriculost $\left.{ }^{\star}\right):$ ti,ab

\#6 depps

\#7 \#4 or \#5 or \#6

\#8 MeSH descriptor: [Cerebrospinal Fluid Shunts] explode all trees

\#9 MeSH descriptor: [Equipment Design] explode all trees

\#10 MeSH descriptor: [Surgical Instruments] explode all trees

\#11 \#8 or \#9 or \#10

\#12 \#7 or \#11

$\# 13 \# 3$ and \#12 
Cochrane Reviews matching "\#13 - \#3 AND \#12"

Cochrane Database of Systematic Reviews

Issue 2 of 12, February 2020

Cochrane Protocols matching "\#13 - \#3 AND \#12"

Cochrane Database of Systematic Reviews

Issue 2 of 12, February 2020

Trials matching "\#13 - \#3 AND \#12"

Cochrane Central Register of Controlled Trials

Issue 2 of 12, February 2020

Editorials matching "\#13 - \#3 AND \#12"

Cochrane Database of Systematic Reviews

Special collections matching "\#13 - \#3 AND \#12"

Clinical Answers matching "\#13 - \#3 AND \#12

\begin{tabular}{|c|c|}
\hline Embase & 1 exp hydrocephalus/ \\
\hline Ovid: Embase & 2 hydrocephal $^{*}$.ti,ab. \\
\hline$<1974$ to & 31 or 2 \\
\hline \multirow[t]{18}{*}{ date> } & 4 (convention* adj3 (valve* or shunt*)).ti,ab. \\
\hline & 5 (cerebrospinal $^{\star}$ adj3 (valve ${ }^{\star}$ or shunt $\left.\left.{ }^{\star}\right)\right) . t i, a b$. \\
\hline & 6 (cerebro spinal ${ }^{\star}$ adj3 $\left(\right.$ valve $^{\star}$ or shunt $\left.\left.^{\star}\right)\right)$.ti,ab. \\
\hline & 7 (ventriculoperiton* adj3 (valve* or shunt*)).ti,ab. \\
\hline & 8 (ventriculo periton ${ }^{\star}$ adj3 (valve ${ }^{\star}$ or shunt $\left.{ }^{\star}\right)$ ).ti,ab. \\
\hline & 9 (conservativ* adj3 (valve* or shunt*)).ti,ab. \\
\hline & 10 (traditional $^{\star}$ adj3 (valve* or shunt $\left.^{\star}\right)$ ).ti,ab. \\
\hline & 11 (standard $^{\star}$ adj3 (valve* or shunt $\left.\left.^{\star}\right)\right) . t i$, ab. \\
\hline & 12 (programm* adj3 (valve* or shunt $\left.\left.{ }^{\star}\right)\right) . t i, a b$. \\
\hline & 13 (pressure adj3 (valve* or shunt $\left.{ }^{\star}\right)$ ).ti,ab. \\
\hline & 14 (shunt $^{\star}$ adj4 valve $\left.{ }^{\star}\right) . t i, a b$ \\
\hline & 15 DEPSS $^{\star} . \mathrm{ti}, \mathrm{ab}$ \\
\hline & 16 (endosc $^{\star}$ adj3 ventriculost ${ }^{\star}$ ).ti,ab. \\
\hline & 17 (gravitat* $^{\star}$ adj3 value*).ti,ab. \\
\hline & 18 ventriculost $^{\star} . \mathrm{ti}, \mathrm{ab}$ \\
\hline & 194 or 5 or 6 or 7 or 8 or 9 or 10 or 11 or 12 or 13 or 14 or 15 or 16 or 17 \\
\hline & or 18 \\
\hline & 20 exp cerebrospinal fluid shunting/ \\
\hline
\end{tabular}


21 exp equipment design/

22 exp surgical equipment/

2320 or 21 or 22

2419 or 23

253 and 24

26 exp "systematic review"/

27 meta analysis/

28 systematic review ${ }^{\star}$. ti,ab.

29 meta analys ${ }^{\star} . t i, a b$.

30 meta?nalys ${ }^{\star}$.ti,ab.

31 Cochrane.ti,ab.

32 (MEDLINE and CENTRAL).ti,ab.

3326 or 27 or 28 or 29 or 30 or 31 or 32

3425 and 33

35 random*.tw. or placebo*.mp. or double blind ${ }^{\star} . \mathrm{mp}$.

3625 and 35

This strategy was adapted to Elsevier platform. See Differences between protocol and review and line below.

\section{LILACS}

$<1980$ to $12.02 .2020>$

(tw:((mh:(hidrocefalia )) OR (tw:(hidrocefal\$ OR hydrocephal\$)) )) AND (tw:((tw:((tw:((tw:(convencion\$ OR convention\$ OR traditional\$ OR tradicional\$ OR conserv\$ OR program\$ OR standard\$ OR estandar\$ OR cerebrospinal\$ OR cerebro spinal\$ OR cefalorraquid\$ OR cefalo raquid\$ OR ventriculoperiton\$ OR ventriculo periton\$ OR presion\$ OR pressure\$)) AND (tw:(shunt\$ OR valv\$ OR deriv\$))) OR (tw:((tw:(shunt\$ OR deriv\$)) AND (tw:(valv\$ )))) OR (tw:((tw:(endosc\$)) AND (tw:(ventriculost\$)))) OR (tw:((tw:(gravita\$)) AND (tw:(value\$ OR valor\$)))) OR (tw:((tw:(ventriculost\$)))))) OR (tw:((mh:(Equipment Design )) OR (mh:(Surgical Instruments )) OR (mh:(Cerebrospinal Fluid Shunts ()))))

\section{ClinicalTrials.gov}

Hydrocephalus OR hydrocephalia

$<$ Inception to $12.02 .2020>$

ICTRP Hydrocephal*

$<$ Inception to $12.02 .2020>$ 


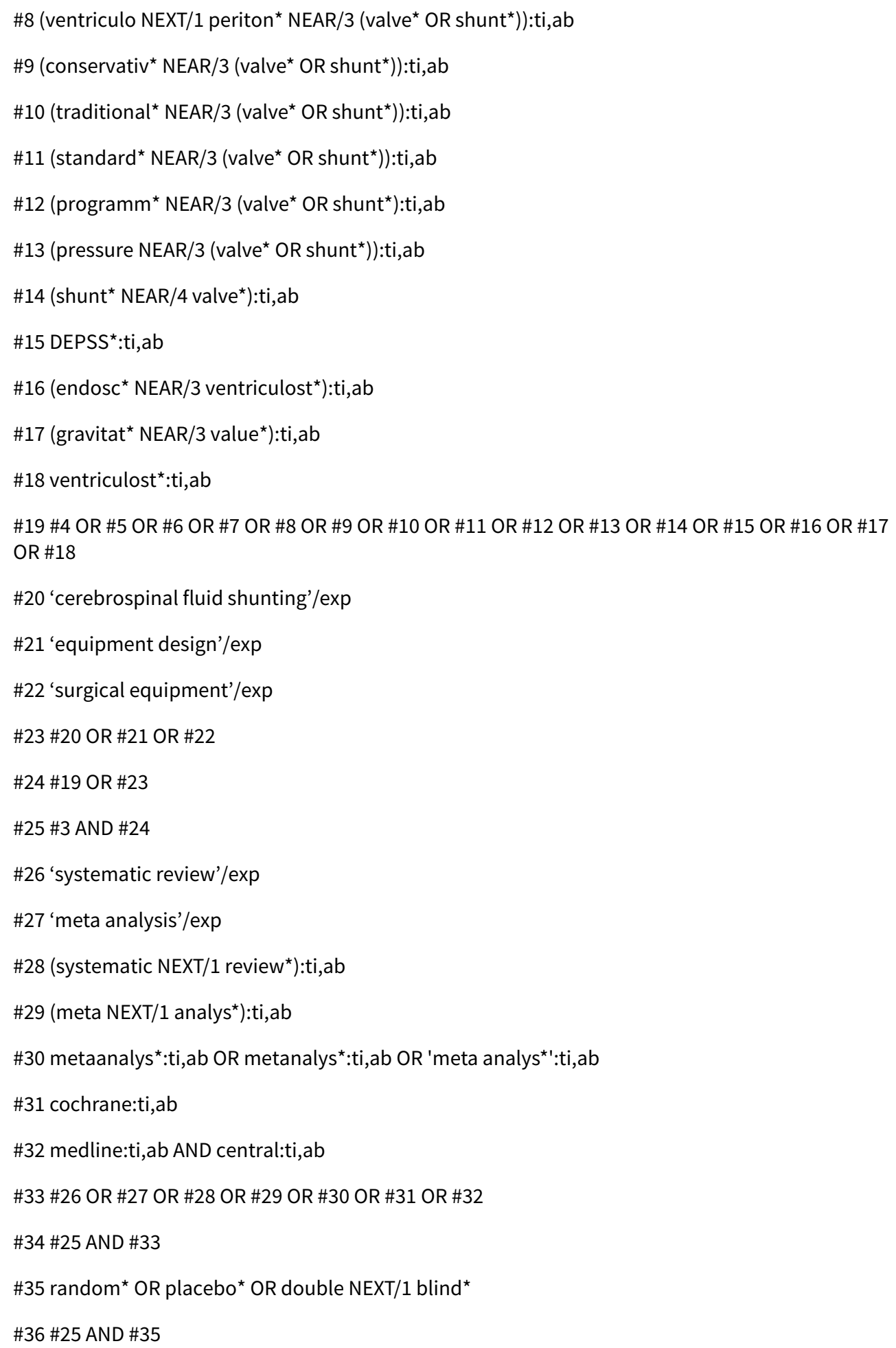




\section{Appendix 2. Abstracts proceedings}

\begin{tabular}{ll}
\hline Conference & Source \\
\hline European Society of Pediatric Neurosurgery, May 2018 & ESPN 2018 \\
\hline International Society of Pediatric Neurosurgery, October 2019 & ISPN 2019 \\
\hline International Society of Pediatric Neurosurgery, October 2018 & ISPN 2018 \\
\hline International Society of Pediatric Neurosurgery, October 2017 & ISPN 2017 \\
\hline
\end{tabular}

\section{H I S T O R Y}

Protocol first published: Issue 7, 2017

Review first published: Issue 6, 2020

\section{CONTRIBUTIONS OF AUTHORS}

SAPM is the contact person with the editorial base and also the clinical specialist author on the review topic. He designed the protocol and was responsible for the final draft of the review.

LIG and JVAF amended the protocol, extracted data and conducted the analysis, drafted the 'Summary of findings' table and wrote the final draft of the review.

VV contributed to the drafting of the protocol and data extraction and analysis of the review.

AC contributed to the methods section.

VG designed and ran the searches, writing the respective methods and results sections.

All authors have reviewed and approved the final version of the protocol.

\section{DECLARATIONS OF INTEREST}

SAPM - none.

VV - none.

LIG - none.

JVAF - none.

$\mathrm{AC}$ - none.

VG - none.

\section{SOURCES OF SUPPORT}

\section{Internal sources}

- Centro Cochrane Instituto Universitario, Hospital Italiano de Buenos Aires, Argentina Provided methodological support for the review

- Paediatric Neurosurgery Service, Department of Paedriatrics, Hospital Italiano de Buenos Aires, Argentina Provided clinical support for the review

- Family and Community Medicine Service, Hospital Italiano de Buenos Aires, Argentina Provided methodological support for the review 


\section{External sources}

- No sources of support supplied

\section{DIFFERENCES BETWEEN PROTOCOLANDREVIEW}

\section{Outcomes}

Since we found an overlap in the causes of shunt failure and adverse events (morbidity leading to shunt revision), we redefined the primary outcomes (and added the timing of outcome measurement) to avoid the overlap and maximise the use of informative data. Furthermore, "mortality related to the procedure" was usually difficult to assess. Therefore, we reported early mortality in order to avoid an inadequate interpretation of the findings and maximise the use of data regarding mortality. The outcomes selected for the 'Summary of Findings' tables were modified accordingly.

\section{Search methods}

The Trials Register of the Cochrane Multiple Sclerosis and Rare Diseases of the CNS Group includes controlled trials comparing alternative forms of interventions used for multiple sclerosis and other demyelinating diseases. Therefore, it was not searched due to the absence of records regarding hydrocephalus.

The Embase search strategy was adapted to the Elsevier platform due to a change in our access to the database. Both the previous strategy (OVID) and the current one are available in Appendix 1. The new strategy does not use filters from the original protocol (Health Information Research Unit (HIRU)).

The search engine for the source of grey literature of the New York Academy of Medicine Grey Literature Report (www.greylit.org) did not allow us to combine search terms for a systematic search strategy.

\section{Synthesis methods}

We added a section on synthesis without meta-analysis in Data synthesis since most of the data were scarce and new guidelines on how to conduct narrative synthesis of quantitative data emerged this last year. Therefore, we were unable to conduct predefined subgroup and sensitivity analyses. Furthermore, we deleted the sentence "If we suspected that missing data are not missing randomly, we performed sensitivity analyses by imputing outcomes to recreate the most extreme possible data sets, one reflecting the best-case scenario for the experimental treatment and the other the worst-case scenario as described in Higgins 2008" since we did not impute data.

\section{'Summary of findings' table}

We added information on the available comparisons and the timing of outcome measurement. 